\title{
Combining Mean Field Calculations with Monte Carlo Simulations for Polymer Gels and Dendrimers
}
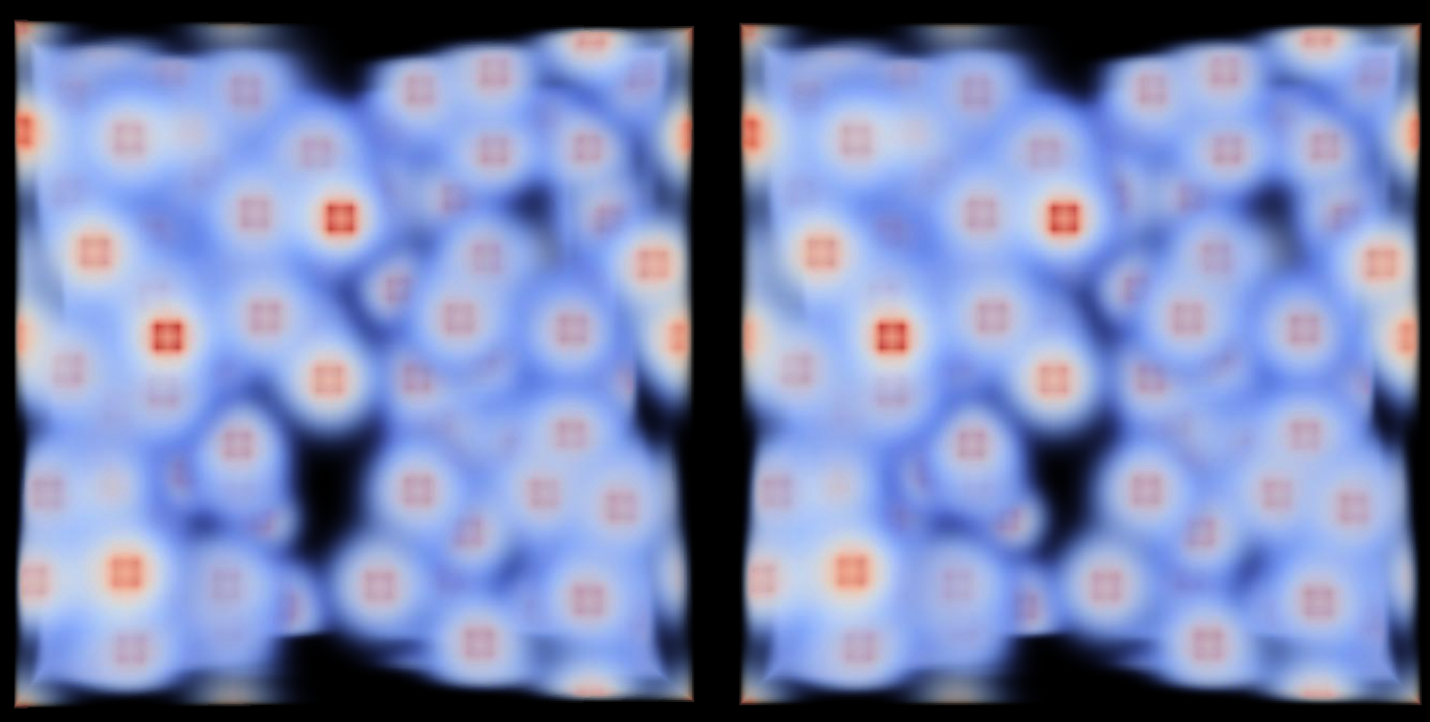

J. P. Bergsma 


\section{Propositions}

1. The interactions between flower-like micelles are not pairwise additive. [this thesis]

2. Scheutjens-Fleer self-consistent field calculations can be too quick to be true. [this thesis]

3. Science is like a Monte Carlo simulation: If the success rate is high, the steps are too small for optimal progress.

4. Publishing in open-access journals improves impact.

5. Yesterdays trash heaps will be tomorrows mines.

6. The net output of the meat industry is waste rather than food.

7. The relative unpopularity of international train travel is partly due to customer-unfriendly ticket sales.

These propositions belong to the thesis:

"Combining Mean Field Calculations with Monte Carlo Simulations for Polymer Gels and Dendrimers"

J. Bergsma

Wageningen, 11th February 2019 


\title{
Combining Mean Field Calculations with Monte Carlo Simulations for Polymer Gels and Dendrimers
}

\author{
J. P. Bergsma
}




\section{Thesis Committee}

\section{Promotor}

Prof. Dr Frans A. M. Leermakers

Personal Chair at Physical Chemistry and Soft Matter

Wageningen University \& Research

\section{Co-promotor}

Prof. Dr Jasper van der Gucht

Professor of Physical Chemistry and Soft Matter

Wageningen University \& Research

\section{Other Members}

Dr Peter Košovan

Charles University, Czech Republic

Prof. Dr Aldrik Velders

Wageningen University \& Research

Dr Ruud van der Sman

Wageningen University \& Research

Dr Joost de Graaf

Institute for Theoretical Physics, Utrecht University, Netherlands

This research was conducted under the auspices of graduate school VLAG (Advanced studies in Food Technology, Agrobiotechnolgy, Nutrition and Health Sciences). 


\title{
Combining Mean Field Calculations with Monte Carlo Simulations for Polymer Gels and Dendrimers
}

\author{
Johannes Pieter Bergsma
}

\section{Thesis}

submitted in fulfilment of the requirements for the degree of doctor at Wageningen University by the authority of the Rector Magnificus, Prof. Dr A. P. J. Mol, in the presence of the

Thesis Committee appointed by the Academic Board to be defended in public on Monday 11 February 2019 at 1:30 pm in the Aula. 
Johannes Pieter Bergsma

Combining Mean Field Calculations with Monte Carlo Simulations for Polymer Gels and Dendrimers

190 pages

PhD thesis, Wageningen University, Wageningen, NL(2019)

DOI: https://doi.org/10.18174/463834

ISBN: $978-94-6343-377-8$ 


\section{Contents}

1 Introduction 1

2 Interactions Between Nodes in a Physical Gel Network of Telechelic Polymers; Self-Consistent Field Calcula$\begin{array}{ll}\text { tions Beyond the Cell Model. } & 17\end{array}$

3 Hybrid Monte Carlo Self-Consistent Field Approach to Model a Thin Layer of a Polyelectrolyte Gel near an Adsorbing Surface

4 A Hybrid Monte Carlo Self-Consistent Field Model of Physical gels of Telechelic Polymers.

5 Coarse-Grained Dendrimers in Good Solvent; Comparison of Monte Carlo Simulations, Self-Consistent Field Theory and a Hybrid Modelling Strategy.

6 General Discussion

Summary

Samenvatting

List of Publications

Bibliography

Acknowlegements

Dankwoord 



\section{CHAPTER 1}

\section{Introduction}

Today's society would be unthinkable without polymers. Because of the wide range of properties they can have, they are used in many applications such as paint, tires, plastic bags, absorbents and thickeners. Some are chemically synthesized, for example: Bakelite, polyurethane, silicones, polystyrene, Teflon, kevlar, and nylon, while others, such as: DNA, chitin, starch, cellulose and rubber, occur naturally.

The defining property of polymers is that they are built up from small molecules, called monomers, that are chemically bound to each other, forming linear, branched or network like structures. Some examples of the monomers from which these polymers can be made together with some of their applications are shown in Figure 1.1. In this thesis we studied two subclasses of these polymers, telechelic polymers and dendrimers, which are also known as star burst polymers.

\section{Dendrimers}

Dendrimers are strongly branched molecules, that are built up from a central segment/branch point. A number of arms is attached to this central segment and at the end of these arms there are branch points from which new arms originate. The dendrimers can be characterized by the number of generations, i.e. the number of branch points encountered going from the centre to the outside, the functionality, which is the number of arms that are connected at a branch point, and the length of the spacer, which is the piece of the arm between the branch points. An example of a dendrimer is shown in Figure 1.2

One of the possible applications of dendrimers is in blood substitutes. Free oxygen carrying heme groups are toxic and are quickly degraded in the bloodstream. When they are encapsulated inside a 

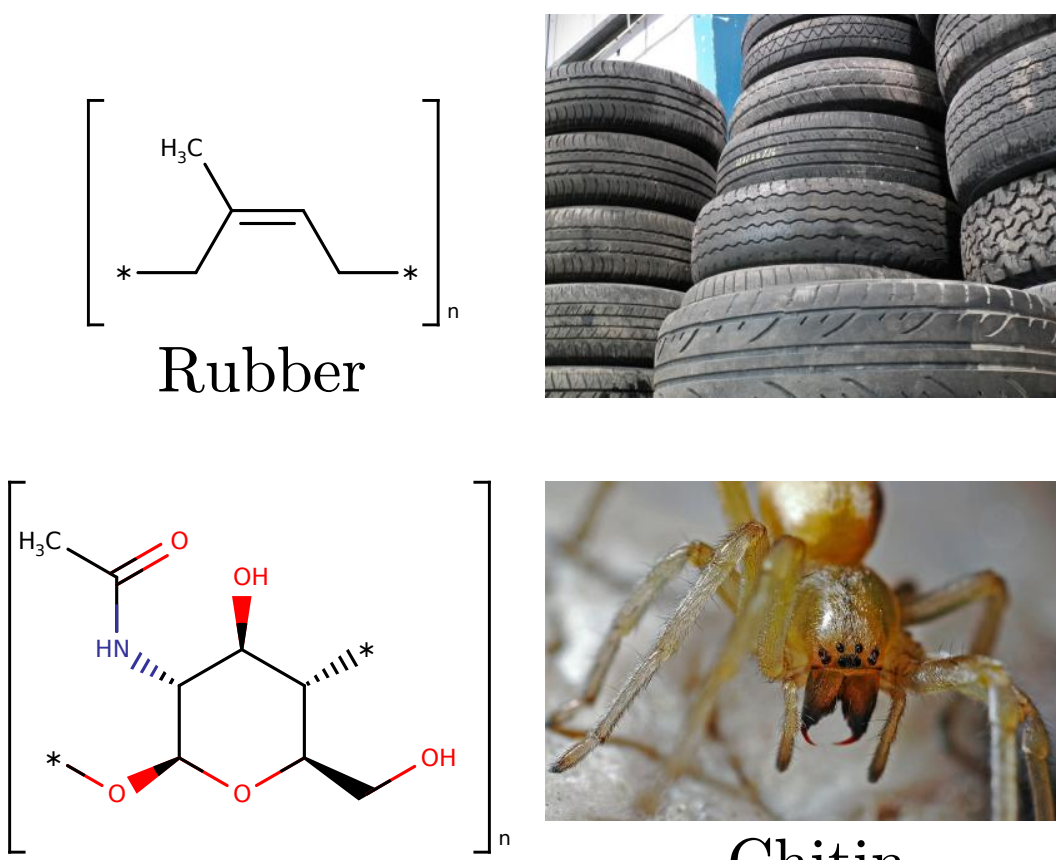

\section{Chitin}

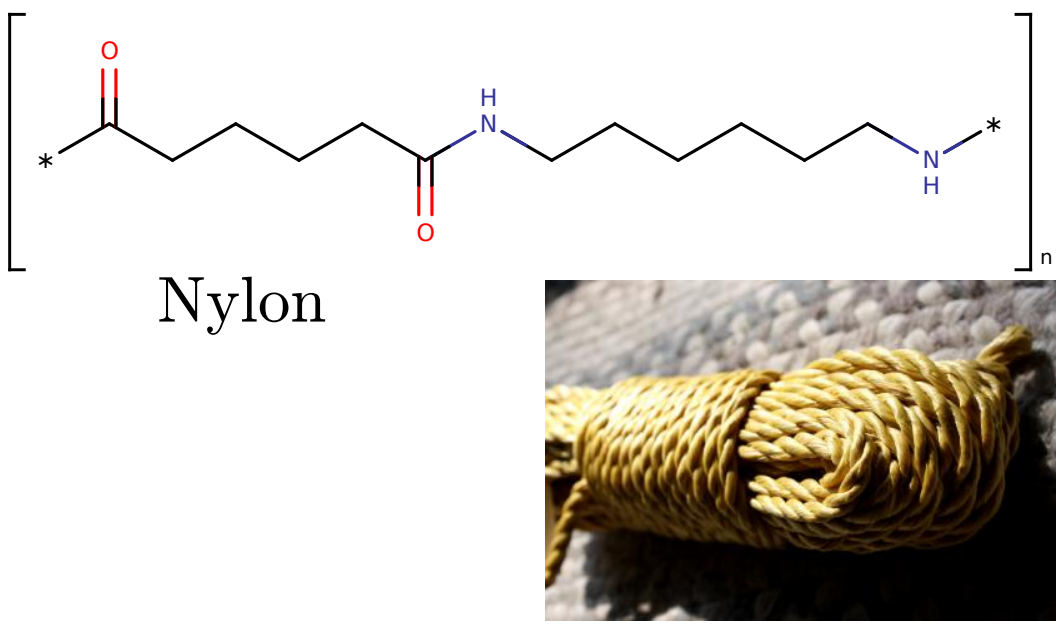

Figure 1.1: Some examples of polymers. On top: polyisoprene which is the main component of rubber. In the middle: chitin which is the main component in the exoskeletons of arthropods. On the bottom Nylon which is used to make fibres for ropes and clothing. 


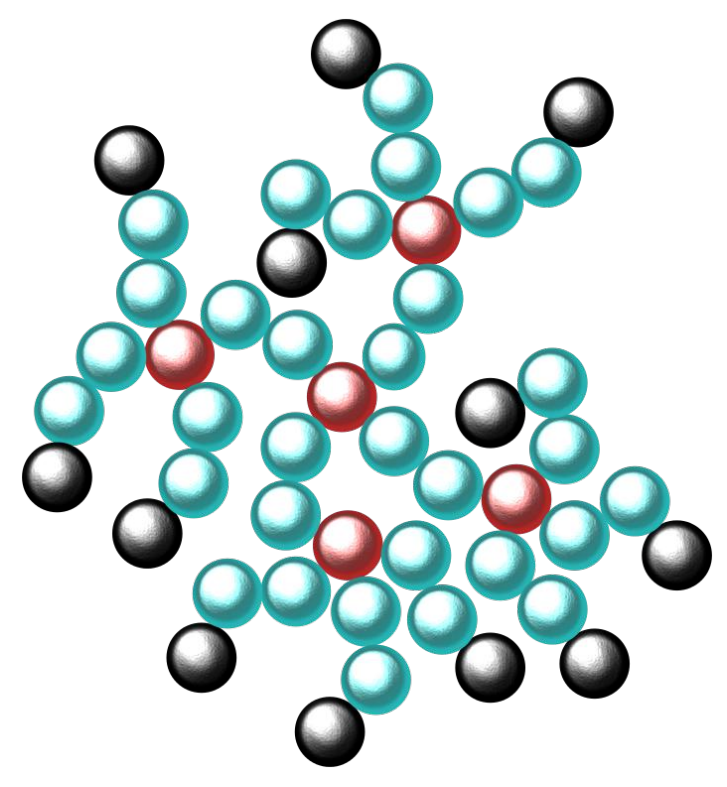

Figure 1.2: An example of a dendrimer with 4 arms per branch point, 2 generations, and spacer length (number of segments in between branch points) of 2 . The spacers are cyan (lightest), the branch points are red and the end-points are black. (darkest)

dendrimer the many branches prevent enzymes from reaching the heme molecules. This reduces the toxicity compared to free heme and prevents enzymes from degrading the heme groups. The dendrimers are however sufficiently porous that small molecules like oxygen can still bind to the heme group.

Another application of dendrimers is in the synthesis of nano particles. Metal ions can bind to the dendrimers. When a reducing agent is added the ions are reduced. Due to the locally high concentration of metal ions in the dendrimer, a metal nano particle is nucleated in each dendrimer. Most of the metal ions in the dendrimer will end up in this nano particle and as a result the nano particles are more mono disperse than with other synthesis techniques. 


\section{Telechelic Polymer Networks}

Telechelic polymers are linear polymers that have end groups that can bind in some fashion. There are numerous ways in which this binding can take place. The most common way to bind is by hydrophobic interactions. ${ }^{112}$ Other ways in which these ends can interact are, for example, by each end having an opposite charge thus forming a so called complex coacervate ${ }^{3 / 4}$, or by binding specifically to each other, a metal ion $\sqrt{5}$, the surface of a particle or a droplet. 8 When the ends of multiple polymers bind together a micelle is formed. The middle blocks form loops sticking out into the solution. These micelles are called flower-like micelles because the loops look a bit like petals, while the ends, in the core of the micelle, form the heart of the flower.

The number of polymers that can bind together in a micelle is limited. The middle blocks get in each others way and prevent more polymers from binding to the micelle. This layer of middle blocks around the centre of the micelle is called the 'corona'.

If two micelles are next to each other, each end of the polymer can be in a different micelle. In that case the polymer forms a 'bridge' between the two micelles. If the micelles are close to each other both loops and bridges can thus be formed, while if they are far apart only loops can be created. When two micelles are close to each other there are therefore more ways to distribute the polymers over the micelles and thus more 'states'. Assuming their energy is the same, each state occurs equally often, the micelles are therefore more likely to be next to each other than far apart. There is thus an entropic attraction between the micelles. If the repulsion between the polymers is small enough and there are enough polymers per micelle, this attraction can become so strong that phase separation occurs and two phases, one with a high polymer concentration and one with a low polymer concentration, are formed. ${ }^{8}$ In this concentrated phase the number of bridges is so high that a volume spanning network is formed. This can also occur for micelles that do not phase separate as long as the concentration is high enough. In this network the cores of the micelles form the nodes and the middle blocks connect these nodes.

Forces can be carried through this network of the polymers and micelles. Most of the volume is still taken up by the solvent and this 


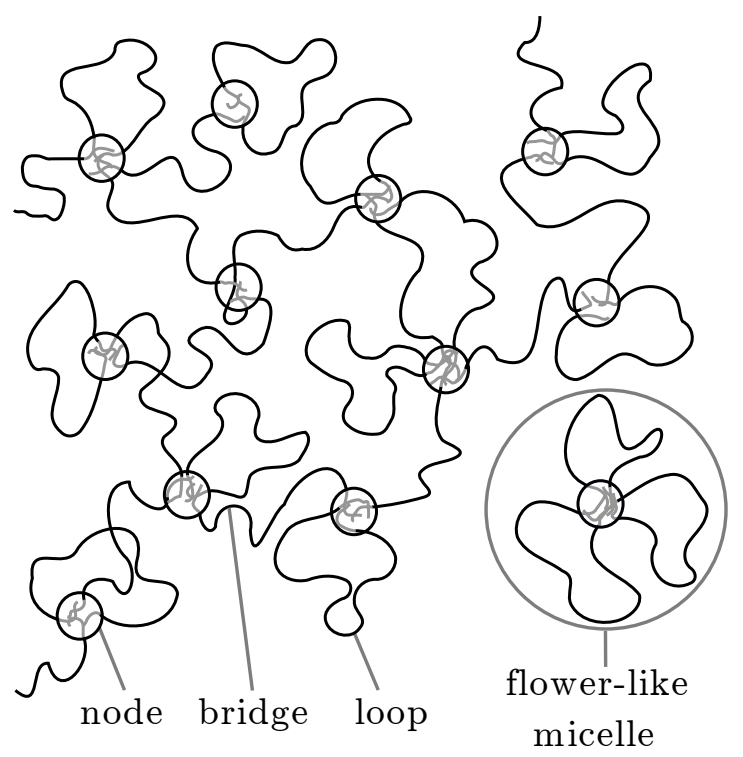

Figure 1.3: A schematic representation of a gel made of telechelic polymers. The circles are the nodes, formed by the micellar cores with the associated end-blocks (indicated by gray lines), connected by the soluble middle blocks (black lines). The telechelics can form loops (both ends in the same node) or bridges (each end in a different node). An isolated flower-like micelle is shown in the lower right corner.

kind of material is called a 'gel', after gelatine, which also forms such a solvent filled network structure. A schematic representation of this polymer gel is given in Figure 1.3 .

The polymer bridges between these micelles behave like springs. If the gel is deformed it takes energy to stretch them and similarly it takes energy to push the loops in the coronas into each other. When the gel is however deformed too much, the end groups of the polymer are pulled apart breaking the network structure. If the binding of the end groups is reversible, i.e. the end groups sometimes spontaneously detach and reattach, the gel will be able to heal itself and regains its full strength after a while.

This kind of behaviour makes it interesting to use these polymers as thickeners in paint. ${ }^{9}$ At the right concentration a weak network is 
formed. When the paint is then applied with a brush this network is broken and the paint becomes liquid. This allows the paint to smooth out and form a flat and shiny surface. On a bit longer time scale the network restores itself and the paint can no longer flow. This way, dripping of the paint is prevented.

Another possible application is drug delivery. ${ }^{10}$ Some drugs are very hydrophobic and are therefore poorly soluble in water. The cores of the micelles can however have a hydrophobic environment and the drugs can be dissolved in these. If a gel with these micelles would be injected in the body it would slowly release micelles with the drug inside them. These micelles can then travel throughout the body to release the drug. An additional advantage is that the micelles are much bigger than the molecules of the drug and would thus be excreted much slower by the kidneys.

Another interesting direction to investigate is the combination of such a telechelic polymer network, whose bonds are reversible, with a second network, which is more flexible. Gels made of two separate networks have been reported to be much tougher than gels made of a single network. ${ }^{111}$ It is hypothesized that in this case the stiffer network is broken upon deformation and in the process absorbs a lot of energy, while the soft network remembers the original shape. This results in a gel with a high toughness. With the telechelic polymers that can bind reversibly, as the brittle part, such a gel could heal itself after being deformed and would maintain its toughness after repeated deformations. Such tough self healing gels could be used as a substitute for damaged tissues, like cartilage, which is also made of a very tough hydrogel.

\section{Project Description}

The goal of my $\mathrm{PhD}$ project was to investigate how the properties of the individual polymers, that form these networks, influence the properties of the gel. Within this project three Phd students worked together. Two of them, Wolf Rombouts ${ }^{12}$ and Małgorzata Bohdan ${ }^{7}$, did experiments in the lab while I worked on computer simulations.

Gosia Bohdan used telechelic polymers with a PEO middle block end capped by hydrophobic or metal binding end groups. She made micelles with a varying ratio of mono- to difunctionalized telechelic polymers 
and studied the effect of this ratio on the rheological properties, such as viscosity and the storage and loss moduli. ${ }^{7}$

She also worked with coated silica particles with polymers with metal binding end groups. ${ }^{6}$ If end groups of polymers from two different particles bind to the same metal ion a bridge is formed just as a hydrophobically end capped polymer can form a bridge by having each end in a different micelle. The behaviour of these coated particles will therefore be similar to micelles of telechelic polymers.

Wolf Rombouts used recombinant proteins, which are genetically engineered proteins, in which parts of natural proteins are combined to create new proteins with different structural properties. He made gels in which a soft flexible network was formed by collagen like proteins, which had end groups that can bind to each other forming triple helices. The second component was formed by silk like proteins which can stack to form long fibres which can form a brittle network. The combined network fractured at higher stresses and at larger shear than the individual components. $\frac{13}{13}$ They attributed this change to the bundling of the silk like fibres due to the depletion interaction caused by the other network.

He also studied a mixture of two different fibres and made polymers which combined the silk and collagen block. As the collagen triple helix unfolds at high temperature this allowed the tuning of the number of extra crosslinks in the network of the silk like polymers. 14

\section{Simulation Methods}

The micelles and polymers are too small to be seen with an ordinary microscope. An electron microscope can visualize objects of the size of our micelles. The polymers themselves would, however, still be too small to be seen. It would also be difficult to study the gels while they are sheared, as the samples would need to be frozen instantaneously to preserve the detailed structure. Other techniques such as X-ray and neutron scattering can give information about the structure on such small length scales but the results can be difficult to interpret. With computer simulations there is no problem with observing the detailed structure of the gel.

In computer simulations real objects are represented by properties 
such as their position/orientation, their shape and size, and how they interact with other objects. In case of molecular systems we often assume that the individual particles are point like so their only relevant property is how they interact with the other particles. These molecular computer simulations can roughly be divided up in three groups: molecular dynamics simulations(MD), Monte Carlo simulations (MC) and mean field models.

\section{Molecular Dynamics}

With MD simulations the particles are moved based on Newtons equations of motion and the forces the particles exert on each other. In this case the particles follow realistic trajectories which is important for the study of dynamic properties and systems that are out of equilibrium. The simulations can be done in an NVE, NVT or NPT ensemble. Here the letters indicate which quantities are kept constant during the simulation. $\mathrm{N}$ is the number of particles, $\mathrm{V}$ the volume, $\mathrm{E}$ the energy, $\mathrm{T}$ the temperature and $\mathrm{P}$ the pressure. The time steps the system takes cannot be too big as otherwise the trajectory of the particles is no longer realistic. For the NVE ensemble the steps need to be even shorter to ensure that energy is conserved. The fact that particles follow a realistic trajectory can also be a disadvantage. When the system gets stuck in a local minimum, it may take a long time before it crosses the barrier. If we are only interested in the equilibrium properties of the system and not in the dynamics/time evolution it is better to use a Monte Carlo method.

\section{Monte Carlo}

With a Monte Carlo method an estimate of a property is made by randomly sampling states of the system and averaging of the value of the property over all states. The Monte Carlo method was first conceived by Enrico Fermi when he studied neutron diffusion. Later Stanislaw Ulam had the same idea when he tried to determine the probability that a particular solitaire game was winnable. He failed to do this with mathematical methods and wondered whether he should have simply played many games while keeping track of the percentage of games he won. He then realized that the problem of how neutrons would 
diffuse and propagate in fission devices could be solved in a similar fashion. Together with John von Neumann they set up the ENIAC computer to randomly generate many individual neutron trajectories using random numbers to determine how far the neutron would travel before it collided with an atom, whether the collision results in fission, scattering or absorption, how many neutrons would be produced in case of fission and what the direction and velocities of the neutrons after the collision would be. They used the outcomes of many of these trajectories to predict the explosive behaviour. 15

This methodology works if the probability with which each state or neutron trajectory is generated is equal to the probability with which it occurs. For many systems we however do not know in advance with which probability a state occurs until after it has been generated. When calculating the average properties of such a system one can of course try to use a weighted average. For many molecular systems the vast majority of the states however have a very low probability of occurring. In that case one could do a simulation without ever sampling the most representable states. To solve this problem Metropolis et al. $\frac{16}{16}$ developed the Metropolis-Hastings algorithm. Metropolis et al. designed it specifically for molecular systems but Hastings ${ }^{17}$ showed it could be applied more broadly.

It starts by choosing a starting state $A_{\text {old }}$. Subsequently, this state is changed such that a new but still correlated state $A_{\text {new }}$ is created. This change or 'move' is accepted based on the probability with which these states occur. If the probability with which the new state occurs $P_{\text {new }}$ is higher than the probability with which the old state occurs $P_{\text {old }}$ the move is accepted while if the probability of occurrence is lower, the move is accepted with a probability $P_{\text {accept }}$.

$$
P_{\text {accept }}=P_{\text {new }} / P_{\text {old }}
$$

If the move is rejected the old state is counted again. This way the evolution of the system is biased in the direction of states that have a high probability of occurring and each state is visited according to it's statistical weight so we can simply average over all samples to get an estimate of a property of the system.

For molecular systems such as those in this thesis the probability 
that a state occurs $P_{A}$ is proportional to the Boltzmann weight.

$$
P_{A} \propto e^{\frac{-U_{A}}{k_{b} T}}
$$

Here $U_{A}$ is the energy of the system in state $\mathrm{A}$ and $k_{B} T$ is the thermal energy a particle has.

Monte Carlo simulations have the advantage that moves do not need to be realistic. Instead the moves can be designed to make the system relax to the ground state quickly and sample as many relevant configurations as possible within a limited amount of computation time.

To make sure that such a move samples the states properly it has to fulfil the balance condition. Frenkel et al. $\frac{18}{}$ clearly explained this by assuming there are infinitely many Monte Carlo simulations. The number of Monte Carlo simulations in each state should be proportional to $P_{A}$, the probability that the simulated system is in state $A$. When we continue these simulations this distribution should not change, hence the rate with which the simulation leaves state $A$ should be equal to the rate with which it goes to state $A$. This is the balance condition. In practice it is easier to prove a stronger condition, namely detailed balance. This means that the rate with which the system goes from state $A$ to state $B$ has to be equal to the rate with which the system goes from state $B$ to state $A$.

For molecular systems such moves usually consist of randomly selecting a particle and moving it over a certain distance or doing some sort of rotation. This distance over which a particle is moved can be much larger than in a molecular dynamics simulation because energy does not need to be conserved. During this move energy barriers can be passed which are normally too high for particles to cross them regularly. A Monte Carlo simulation is thus useful to study the equilibrium structure and properties of a system but, unlike a MD simulation, it does not give insight in the dynamics.

\section{Mean Field Models}

With mean field models one does not try to determine individual particle configurations, instead we determine their distribution based on the average interactions the particles have with their environment. This 
method is even faster because we do not need to sample all the particle configurations individually but instead sample them all at once.

An example of such a mean field theory is the Scheutjens-Fleer self consistent field theory, which is used in this thesis. It was developed in our department at the end of the seventies by Scheutjens and Fleer to describe adsorption of (homo)polymers. 19 21 It is a lattice based model and it describes polymers as a freely jointed chain of beads with each bead occupying a lattice site. The SF-SCF model determines the polymer distribution with the lowest free energy assuming that each polymer interacts with it's average surroundings.

Over the years this method was extended in several directions. The first step was to allow for different segment types in the macromolecules. This was useful for (surfactant) self-assembly 22123 and copolymer adsorption 24 . The method was generalised for branched chains and this, for example, allowed the study of dendrimers and star polymers. 25.27 At first molecules at the interface were equilibrated with chains in the bulk, but restricting segments to specified locations meant that polymer brushes that do not equilibrate with freely dispersed chains could also be modelled. 28129

The mean field averaging originally was performed in planar (lattice) layers. The generalisation to spherical and cylindrical geometries allowed, e.g., for the modelling of the corresponding micelles. ${ }^{22|23| 30}$ Then the idea developed to pin a single chain with a central segment to the centre of a lattice with spherical geometry to study the intramolecular excluded volume effects. This model may be referred to as a cell model and was used to study polymer stars. ${ }^{31}$ It was further realised that the SCF equations could be implemented in two-gradient systems necessary for considering laterally inhomogeneities in polymer adsorption and selfassembly. $\stackrel{32 \sqrt{33}}{ }$ Finally, Hessian-free optimisation techniques allowed the development of SCF computations that develop density gradients in all three directions. In the latter case the mean-field volume fraction should be seen as the (time) average value of the segment concentration. This allows for the modelling of, e.g., the gyroid phase in block copolymer micro-phase segregation. 34

The speed gained by using mean field models however comes at a certain price. Part of the effects of correlations are lost and fluctuations in the system cannot be observed. This is further discussed in chapter 5. 


\section{Modelling Considerations for Telechelic Polymer Micelles}

Not all methods strictly fall in these categories, some are hybrids, such as Car-Parrinello Molecular Dynamics 35 , where the atomic nuclei are moved with molecular dynamics while the electrons are treated in a mean field way, and the hybrid method described in chapter 3,4 and 5.

In principle techniques such as Car-Parrinello Molecular Dynamics ${ }^{35}$, which I used during my internship at the University of Amsterdam, can simulate the gel in full detail. It would however take a huge amount of computer time to simulate even a small piece of gel on the time scale on which the rheologically interesting behaviour takes place. We therefore need to coarse-graine the gel, which means that multiple particles are grouped together and simulated as a single particle. There are various levels to which one could coarse-grain from single atoms, to monomers, polymer segments, whole polymers and finally entire micelles. Ideally, we would coarse-grain to as high a level as possible as this allows us to simulate larger volumes of the gel on longer time scales.

In simulations it is usually assumed that all interactions are pair wise additive, i.e. the interaction between two particles does not depend on the position of other particles around them. For many types of interaction that occur between coarse-grained particles, such as van der Waals, DLVO ${ }^{\sqrt{66}}$ and depletion forces ${ }^{37}$, there are however also contributions from many body terms.

It is therefore far from certain that the micelles can be modelled as a single particle with pairwise potentials. Especially when one considers that if two isolated micelles approach each other closely the loops in between them can move out of the way, while if they are already surrounded by other micelles this is not possible and the loops are pushed into each other. One would therefore expect that the repulsive force between them is stronger, when they are surrounded by other particles.

I decided to test this hypothesis by measuring the interaction potential between the micelles with a varying number of neighbouring micelles using the Scheutjens Fleer self-consistent field model(SF-SCF). The results of the calculations of the interaction potentials are described in chapter 2 .

We already give away here that we found that this interaction potential is not pair wise additive. This meant that a model in which 
the micelles are coarse-grained as single particles and interact through pairwise potentials would not give a reliable description of the gel. We therefore had to look for a different method.

\section{Monte Carlo SF-SCF Hybrid for Gels of Telechelic Polymers}

The SF-SCF theory can describe a relatively large piece of gel within limited computation time. As we have previously stated, the SF-SCF model however does not show fluctuations and neglects part of the correlations. These fluctuations have an effect on the properties of the gel. My supervisor Frans Leermakers therefore wondered whether it was possible to combine the Monte Carlo method with the SCF theory and incorporate the fluctuations in the gel in this way. With this method the cores of the micelles are moved with a Monte Carlo method, while the distribution of the polymers which are anchored to the cores is determined with the SCF theory.

We hoped that this would introduce the needed fluctuation in the structure of the gel while still keeping the computation limited.

In chapter 3 a quick preliminary study is described, of a gel, made of charged telechelic polymers adsorbed on a wall. We wrote this chapter for a festschrift in honour of Herman P. van Leeuwen who worked on diffusive gradient thin films (DGT) and diffusion equilibrium in thin films(DET). These techniques are used to measure the concentration of free metal ions in natural waters. In both cases there is a layer of polymer gel in which the metal ions can penetrate. In DGT films there is also a resin underneath which works as a sink for the metal ions. For both techniques the amount of absorbed metal ions scales with the average free metal concentration. It was however discovered that small humic acid particles with bound metal ions could also penetrate

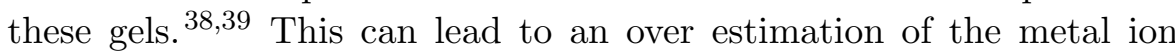
concentration. It is therefore important to know how porous these gels are and how particles are absorbed into them. Due to time constraints we were not able to simulate a large enough part of the gel to answer these questions, but we do show a proof of principle.

Later we did a more extensive study of a gel of neutral telechelic polymers. In this study we varied the number of polymers per micelle, the solvent quality and the polymer concentration and determined the 
structural properties of these gels. The results from these simulations are described in chapter 4 .

\section{Hybrid SF-SCF Monte Carlo Model of Dendrimers}

Introducing the Monte Carlo moves successfully introduced fluctuations into the SF-SCF theory. I wondered whether it would also be possible to use it to introduce some of the correlations back into the SF-SCF theory. Because the SF-SCF is a mean field theory it calculates the interactions based on the average concentration around the segments. Locally the concentration is however higher because the segment is connected to its nearby neighbours. The self-consistent field theory thus underestimates the interactions that polymers have with themselves. It however overestimates the excluded volume interactions between separate polymer chainss. Because the positions of segments in a polymer chain are correlated their excluded volumes overlap and their total excluded volume is thus smaller. This may explain why the average size of a polymer coil as a function of the number of segments $N$ scales with $N^{0.640}$ for the meanfield model while for real polymers it scales with $N^{0.588} \underline{41 / 42}$ By localising some of the segments of the polymer the correlations between the polymer segments are reintroduced into the self-consistent field model. This should result in better scaling behaviour and a better treatment of the excluded volume interactions.

As there are many arms in a dendrimer there will be a lot of excluded volume interactions between these arms. Therefore the effect of the introduction of the localized segments should have a stronger effect on the size of a dendrimer than for a polymer. We therefore chose them as a model system. For a functionality of 3 there is already quite some literature but for dendrimers with higher functionalities of 4 and 5 there is little information and our simulations can still give some useful data about their size and density distribution.

In contrast to the gels of telechelic polymers the bonds in a dendrimer are chemical rather than physical, each spacer is therefore connected to two distinct branch points. We therefore have to calculate the density separately for each spacer. As each spacer can only be in a small part of the total volume, we have introduced a separate sub box for each spacer. This way computation time can be saved. 


\section{Overview Chapters}

\section{Chapter 2; Interactions Between Nodes in a Physical Gel Network of Telechelic Polymers; Self-Consistent Field Cal- culations Beyond the Cell Model.}

In this chapter we investigated the interaction potentials between micelles/ nodes as a function of the number of polymers per micelle and the solvent quality. We did this for varying particle configurations, namely: (i) two isolated nodes, (ii) nodes positioned on a line (iii) a central node surrounded by its neighbours in simple cubic ordering, and (iv) a central node in a face centred cubic configuration of its neighbours. For the first three we used a simple cubic lattice while for the latter a Face centred lattice was introduced in the SF-SCF model. We also compared these results with those from the cell model.

\section{Chapter 3: Hybrid Monte Carlo Self-Consistent Field Ap- proach to Model a Thin Layer of a Polyelectrolyte Gel near an Adsorbing Surface}

Here we described our first experiments with the hybrid of the SFSCF method with a Monte Carlo method. We simulated a small piece of surface bound polyelectrolyte hydrogel. We determined the density profile and found that the adsorbed gel was in equilibrium with a dilute phase.

\section{Chapter 4: A Hybrid Monte Carlo Self-Consistent Field Model of Physical Gels of Telechelic Polymers.}

In this chapter we used the hybrid Monte Carlo SF-SCF method to do a more extensive study of gels made of telechelic polymers. We varied the number of polymers per micelle/node, the polymer concentration and the solvent quality and determined the radial distribution function, the compressibility and the structure factors. We also compared the outcomes of this hybrid Monte Carlo SF-SCF method with a simpler Monte Carlo model where we coarse-grained the micelles as a single particle and used pair potentials based in the interaction potentials we determined in chapter 2 . 
Chapter 5: Coarse-Grained Dendrimers in Good Solvent; Comparison of Monte Carlo Simulations, Self-Consistent Field Theory and a Hybrid Modelling Strategy.

Here we investigated whether localizing some of the segments in a molecule and moving them with a Monte Carlo scheme would improve the way excluded volume interactions are treated in the self-consistent field model. Dendrimers were used as a model system, as excluded volume interactions play an important role in these molecules. We compared the new hybrid method with the localized segments with the classical SF-SCF models and a more detailed Monte Carlo model. We did this for dendrimers with a generation number ranging from 1 to 7 , a functionality ranging from 2 to 5 and spacer lengths of 20 and 50 segments. 


\section{CHAPTER 2}

\section{Interactions Between Nodes in a Physical Gel Network of Telechelic Polymers; Self-Consistent Field Calculations Beyond the Cell Model.}

Triblock copolymers, with associative end-groups and a soluble middle block, form flower-like micelles in dilute solutions and a physical gel at higher concentrations. In a gel the middle blocks form bridges between domains/nodes that contain the ends. We combine the self-consistent field theory with a simple molecular model to evaluate the pair potential between the nodes. In this model the end-groups are forced to remain in nodes. When the distance between the centres of the nodes is approximately the coronal diameter, loops can transform into bridges, and the pair potential is attractive. Due to steric hindrance, the interaction is repulsive at smaller distances. Till now a cell model has been used wherein a central node interacts through reflecting boundary conditions with its images in a spherical geometry. This artificial approach to estimate pair potentials is here complemented by more realistic three-gradient SCF model. We consider the pair interactions for (i) two isolated nodes, (ii) nodes positioned on a line, and a central node surrounded by (iii) a simple cubic ordering or (iv) a face centred cubic ordering of its neighbours. Qualitatively, the cell model is in line with the more refined models, but quantitative differences are significant. We also notice qualitative differences for the pair potentials in the specified geometries, which we interpret as a breakdown of the pairwise additivity of the pair potential. This implies that for course-grained Monte Carlo or molecular dynamics simulations the best choice for the pair potentials depends on the node density.

This chapter was originally published as: "Interactions Between Nodes in a Physical Gel Network of Telechelic Polymers; Self-Consistent Field Calculations Beyond the Cell Model", J. Bergsma, F. A. M. Leermakers and J. van der Gucht, Physical Chemistry Chemical Physics, 2015, 17, 9001-9014 


\section{Introduction}

Triblock copolymers, with associative end-groups and a soluble middle block, are often referred to as telechelic polymers as they can connect to two different parts. The end-groups can, for example, associate by solvophobic interactions ${ }^{1 / 2}$, charge interactions ${ }^{3 / 4}$ or complexation with metal ions. $\frac{517}{17}$ These ends form the cores of what may be called micelles. The solvophilic polymeric middle blocks form the corona of these micelles. $\frac{43}{3}$ The steric hindrance between the chains in the corona prevents macroscopic aggregation, and micelles with a limited number of polymers are formed. ${ }^{44}$ By mixing the telechelic polymers with a cosurfactant, hybrid micelles can be formed and the average number of polymers per micelle can be adjusted. ${ }^{[145}$ In dilute solutions both ends of the polymers have to be in the same core and a loop is thus formed by the polymer. Because these structures look somewhat like a flower, with the polymer loops as the petals and the micellar core as the heart, they are called flower-like micelles.

When the concentration is increased, the distance between the micelles is reduced and the coronas start to overlap. A loop can then transform into a bridge, thereby connecting two micelles. 2 Of course this can only happen when the anchor energy of the ends is sufficiently low to be overcome by the thermal energy. The possibility to form bridges increases the number of polymer conformations and thus the conformational entropy. This creates an attractive force. $\stackrel{44}{ }$ When the average number of bridges per micelle is larger than unity, a volume spanning network can be formed.1 The solution then becomes a gel. Such behaviour is found for the telechelic polymer systems mentioned above. Generically the regions in which the chain ends associate may be referred to as nodes. Similar to chemically crosslinked gels, such associative networks respond elastically upon deformation, but flow at larger time scales. $\frac{8}{}$ A schematic representation of this gel is shown in Figure 2.17. In such gel it is the osmotic pressure in the coronal region, i.e. the steric hindrance between solvated corona chains, that keeps the nodes apart. When the number of bridges per node is sufficiently high, the bridging attraction becomes so strong that phase separation occurs. ${ }^{8}$ In this case the polymer gel coexists with an excess solvent phase with a relatively low polymer concentration. 

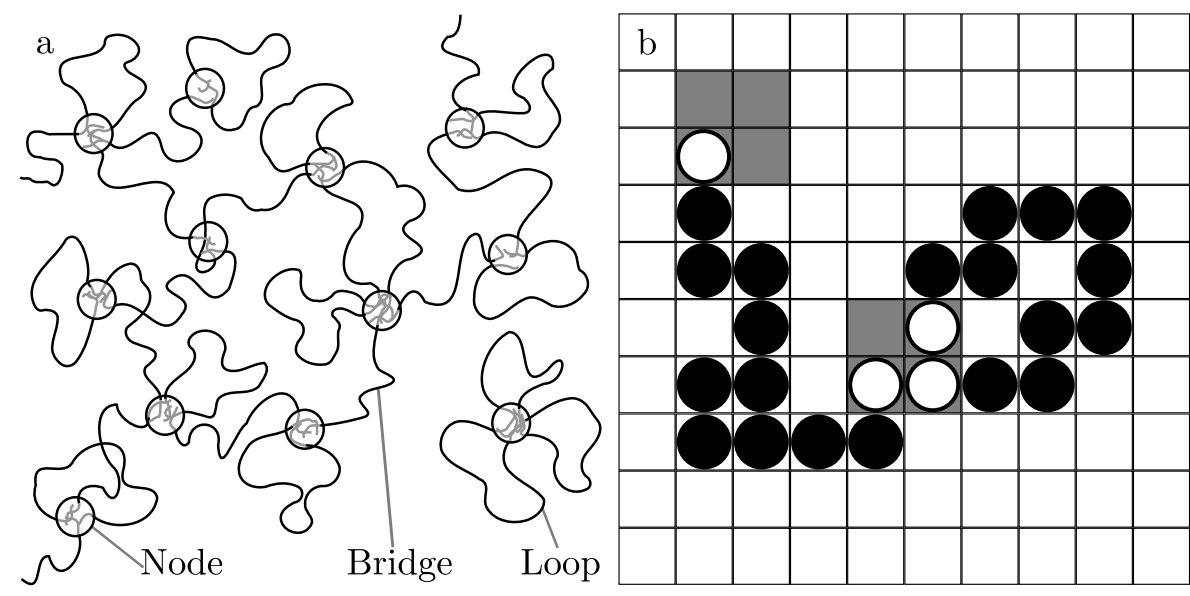

Figure 2.1: a: A micellar network formed by telechelic polymers. The circles are the micellar core, with the grey lines as the associative endgroups and the black line as the soluble middle block. The telechelics can form loops (both ends in the same micelle/node) or bridges (ends are in different micelles/nodes). An isolated flower-like micelle is shown in the lower right corner. b: Two polymer configurations, a loop and a bridge (closed circles), on the simple cubic grid with its ends (open circles) constrained within nodes of 2 by 2 lattice sites (grey).

Gels made of these telechelics have a wide range of applications. They are, for example, used to improve the rheological properties of paints, as a gel material for gel electrophoresis ${ }^{46}$ and are envisioned as carrier material for slow drug release. ${ }^{10}$ As the binding of the endgroups is reversible the gels have the property to heal themselves when they are damaged. $\underline{47 / 48}$

As already elaborated above, there have been many experimental studies on these systems. The number of theoretical counterparts is, however, limited, basically because the system poses significant theoretical challenges. One way to gain additional insights in the physical properties of such systems is to use computer simulations.

As it is virtually impossible to take all degrees of freedom of the polymers explicitly into account, it is necessary to use a so-called coarse- 
grained model. For example by coarse- graining the polymer as a chain of beads, or by modelling a micellar object as a single particle with appropriate pairwise additive interactions between them. $\stackrel{49}{ }$ To implement the latter method, one needs to determine the free energy of interaction between the nodes. This quantity is not easily extracted from MonteCarlo, or molecular dynamics computer simulations.

A solution to this problem is to calculate the pair potential using a self-consistent field (SCF) theory, as done by Sprakel and coworkers. $\frac{49}{4}$ They used the Scheutjens-Fleer version of the self-consistent field theory(SF-SCF) and modelled one flower-like micelle, with a core/node in the centre of a spherically symmetric system with reflecting boundary conditions on the outside; the so-called cell model. Subsequently they recorded the free energy of the system as a function of the cell size. There is however an obvious problem with determining the pair potential from this free energy. The reflecting boundary conditions imply that images of the central node are encountered in all directions and it is unclear how to calculate a realistic pair potential from this unrealistic particle ordering. Sprakel and co-workers tried to solve this by dividing the free energy by twelve. Because a hexagonal ordering has the greatest number of nearest neighbours, twelve, and is thus most similar to the cell model. It is thus expected that this gives the best estimate for the pair potential.

The first goal of this chapter is, therefore, to investigate the suitability of the cell model to obtain pair potentials. We do this by comparing the pair potential calculated with the cell model to that of a pair of nodes on a 3D grid. The advantage of using a 3D grid is that we can model realistic particle configurations, although it takes far more computation time than the cell model. The configurations for which the two pair interactions are compared are: an isolated pair of nodes (IP), a string of Nodes on a line (NoL), and nodes in a simple cubic (cubic primitive: $\mathrm{cP}$ ) or face centred cubic (FCC) arrangement. The pair interactions are further compared for various lengths of the soluble block $N$, number of polymers per node $f$ and solvent qualities $\chi$.

The interactions between nodes with triblock copolymers are largely determined by the entropic effect of loop to bridge transitions and the steric repulsion due to the compression of coronal chains. How the associating end-groups are held together is less important. Therefore we 
simply specify nodes with a predefined volume in which the associative ends of the polymers have to reside. The telechelics thus either form a loop, starting and ending on the same node, or a bridge, when ending on a different node than the one they started from. We assume that the binding of the end-groups is so strong that the number of free ends is negligible and thus disregard the option that a loose tail is formed, even though some free ends must exist for the gel to relax. This model can be implemented in a cell model, but also for the $\mathrm{cP}$ and $\mathrm{FCC}$ lattice.

The primary result in the SCF calculations is the free energy of interaction per node $\Delta F(d)$ as a function of the distance to the nearest neighbour $d$. As the number of neighbours is different in each geometry, we need to extract an effective pair potential $\Delta F_{12}(d)$ from this free energy of interaction to be able to compare the different geometries. Assuming that the pair interactions are pair wise additive, we find $\Delta F_{12}(d)$ by fitting a pair potential such that the sum of the pair interactions of the central node between all contributing node pairs is the same as the free energy of interaction $\Delta F(d)$ for each value of $d$.

The second goal is to determine whether the assumption that the pair potential is pairwise additive is correct. From the interaction between two isolated nodes (IP), we know the classical pair potential and we can compare this with the pair potential $\Delta F_{12}(d)$ found for the other interaction configurations (NoL, cP, FCC) as well as for the cell model. When pairwise additivity is applicable, all the pair potentials for the different interaction geometries should match. For weak interactions between the nodes (large node distances), the total number of polymer configurations is changed only by a little. In this limit we expect that pairwise additivity is strictly obeyed. For strongly interacting nodes, however, the pairwise additivity will likely break down.

\section{Self-Consistent Field Theory and the Molecular Model}

In this section, a brief introduction is given to the SF-SCF theory of Scheutjens and Fleer. $\frac{19|20| 24}{2}$ The theory was originally designed for the case of polymer adsorption 2012150,53 , but found many applications in other fields, such as surfactant self-assembly. $22|33| 53$ 
The target is to find the volume fraction profiles $\varphi_{X}(\mathbf{r})$, for any segment type $X$ at coordinate $\mathbf{r}$, such that the total free energy is minimal. To this end, space is divided up in discrete lattice sites. The lattice sites are organised depending on the symmetry in the system. For the simple cubic and the FCC lattice, parameters are allowed to vary in three dimensions. Then all $x, y$ and $z$ coordinates are specified. One can also recognise lattice layers and average the volume fractions within each layer. Then density gradients only occur in the remaining (e.g. radial) direction, as in the cell model.

Hence, for the simple cubic $(\mathrm{cP})$ and face centred cubic lattice (FCC), the lattice sites are referred to by $\mathbf{r}=(x, y, z)$ coordinates, $x=1,2, \ldots M_{x}, y=1,2, \ldots M_{y}, z=1,2, \ldots M_{z}$, with periodic boundary conditions in all directions. This is implemented by equating densities just outside the box to its periodic neighbour, e.g. $\varphi\left(M_{x}+1, y, z\right)=$ $\varphi(1, y, z)$.

For the simple cubic lattice, which was used for the isolated pair (IP), nodes on a line (NoL), and the simple cubic (cP) configurations, a lattice site at coordinate $(x, y, z)$ has neighbours at $(x+1, y, z),(x-$ $1, y, z),(x, y-1, z),(x, y+1, z),(x, y, z+1),(x, y, z-1)$ and the faces on the lattice sites are at $90^{\circ}$ angles.

An FCC lattice was used for the face centred cubic (FCC) configuration of nodes. In the FCC lattice, a lattice site has 12 nearest neighbours. A lattice site at coordinate $(x, y, z)$ has neighbours at $(x+1, y, z)$, $(x-1, y, z),(x, y+1, z),(x, y-1, z),(x-1, y+1, z),(x+1, y-1, z)$, $(x, y, z+1),(x, y-1, z+1),(x-1, y, z+1),(x, y, z-1),(x, y+1, z-1)$, $(x+1, y, z-1)$. The $x, y$ and $z$ axis are now at an angle of $60^{\circ}$ to each other. The simulation box is thus a parallelepiped with periodic boundary conditions.

The cell model has a spherically symmetric geometry with lattice sites arranged in concentric layers numbered $r=1,2, \cdots, M_{r}$, with layer $r=1$ at the centre. The number of lattice sites in each layer grows with the layer number as $L(r) \sim r^{2}$. The mean field approximation is applied within each lattice layer, which means that the content of all lattice sites in a given layer is identical. We have used reflecting boundary conditions by setting all quantities at $r=M_{r}+1$ equal to that in layer $r=M_{r}$. The reflecting boundary condition implies that the distance between two nodes is $d=2 \times M_{r}$. It must be stressed that the 
reflecting boundary conditions are an artificial means to implement the surrounding nodes around a central one and the number of neighbouring nodes varies with the size of the cell model. For the cell model it is also not defined how many neighbouring lattice sites each lattice site has. With the simple cubic lattice in mind we have chosen to have six neighbours for each lattice site, one in the layer above, one in the layer below and four in the same layer. In practice one also needs to take the curvature into account such that the ratio between the number of neighbours in the layer above and below is the same as the ratio of the surface areas of these layers. ${ }^{[54}$ Below we will focus on the three-gradient SCF approach because this information is not readily available in the literature. The one-gradient equations can be derived from the threegradient ones and therefore we have reduced the amount of detail for the cell model.

Within the SCF theory molecules consist of a number of segments and each segment has the volume of one lattice site. These segments can be of different types and thus have different properties reflected in the interaction parameters. The polymer molecules are represented by a chain of freely jointed segments numbered $s=0,1,2, \cdots, N+1$. Segments $s=0$ and $s=N+1$ are the associative end-groups. The middle $N$ segments form the water-soluble middle block. Subsequent segments, along the chain, have to be on neighbouring lattice sites, but can go in any direction that is consistent with the lattice geometry. The freely jointed chain can therefore cross itself or fold back on itself. This is partially corrected for by imposing an incompressibility constraint, which means that the sum of the volume fractions, of the polymer and the solvent, in each lattice site is exactly one. The segments have to be about a Kuhn length in size for the polymer to be able to fold back. For flexible polymers, like PEO, the length of a segment should thus corresponds to roughly 0.5 to $1 \mathrm{~nm}$.

We want to simulate networks of polymers with associative endgroups. To do this in a computationally inexpensive way, we have simply defined small volumes, called nodes, in which the two terminal segments of all polymers $s=0$ and $s=N+1$ are constrained to be. This is depicted schematically in fig. 2.1 b. For the $\mathrm{cP}$ and the FCC lattice the nodes are small cubes of $3 \times 3 \times 3$ lattice sites. A chain can choose to put the terminal segments in the same node forming a loop, 
or place the terminal segments in two different nodes forming a bridge. An illustration of two polymer configurations on the simple cubic lattice is shown in Figure 2.1b.

In the cell model the terminal segments are confined to a sphere with a radius of two layers at the centre of the coordinate system. As long as the polymer does not cross the reflecting boundary, a loop is formed. If the polymer crosses the boundary once, a bridge is formed. If the polymer crosses the reflecting boundary more than once, it is unclear whether a loop or bridge is formed (there is a superposition of the two cases).

The micelles that are formed by the nodes and polymers can be characterized by the length of the soluble middle block $N$, the number of polymers per node $f$ and the solvent quality for the polymer segments. This solvent quality is specified by the $\chi$ parameter which is defined as the energy required to exchange a solvent and a polymer segment from the pure phases in units $k_{B} T$, just as in the Flory-Huggins solution theory. ${ }^{[55}$ The interactions are accounted for by the usual Bragg-Williams approximation.

We investigated the effect of these characteristic properties of the nodes on the interaction energy between an isolated pair of nodes and for a single node with its mirror images in the cell model. The solvent quality was varied from theta conditions $\chi=0.5$ to a good solvent $\chi=0$. The number of chains per node ranged from $f=1, \cdots, 10$. The polymer length was varied from $N=12$ to $N=500$ for the cell model and from $N=12$ to $N=100$ for the isolated pair. Each segment is roughly a Kuhn segment long. For a flexible polymer like PEO a Kuhn segment is roughly two monomers. ${ }^{56} N=50$ thus coincides with 100 PEO monomers and a middle block weight of $4400 \mathrm{u}$. This falls within the range of middle block weights that are studied experimentally which ranges from about 2000 to $35000 u$. $\frac{112|4| 8|46| 48}{12}$

To determine whether the interactions are pairwise additive we have calculated an effective pair potential for different node configurations: An isolated pair of nodes (IP), a string of nodes on a line (NoL), a simple cubic configuration (cP) and a face centred configuration (FCC) $d$. 

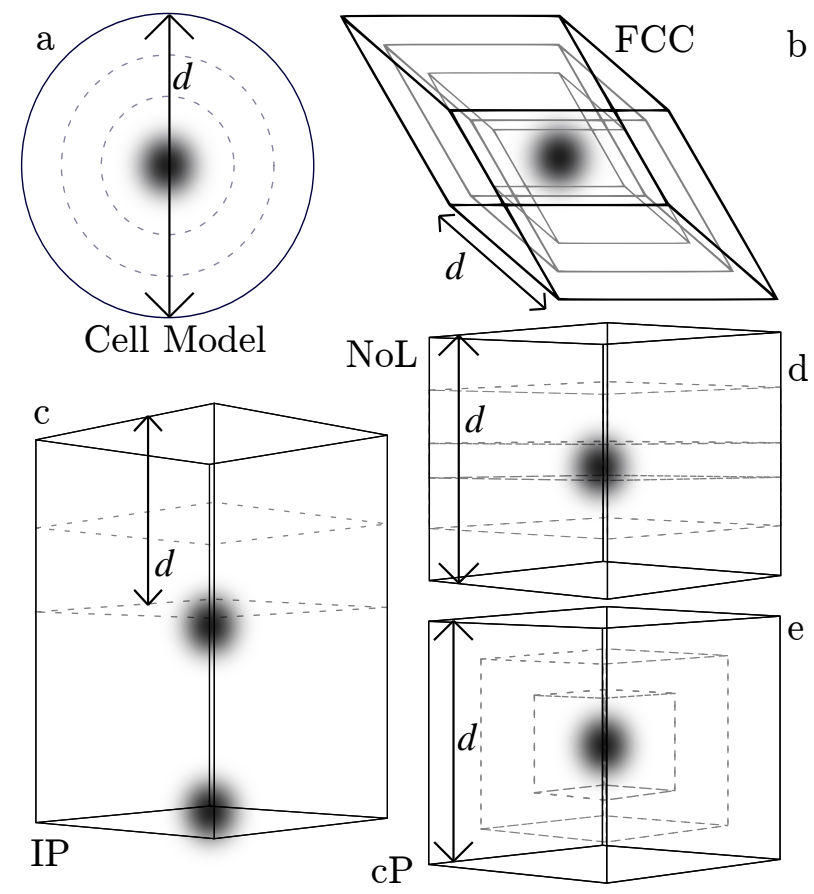

Figure 2.2: Overview of how the distance between the nodes was varied for the different node geometries. The grey (dashed) lines indicate smaller box sizes. The grey regions in the middle represent the node with the polymers around it. a) the cell model b) the FCC configuration c) an isolated pair (IP) d) a string of nodes on a line configuration $(\mathrm{NoL})$ e) a cubic $(\mathrm{cP})$ configuration. Periodic boundary conditions are used except for the cell model where a reflecting boundary is used.

As illustrated in Figure $2.2 \mathrm{c}$ the isolated pair was modelled by placing two nodes in a rectangular box far enough apart to not interact. By reducing the number of layers in the appropriate direction, the distance through the periodic boundary between the nodes is reduced until both nodes touch each other. For the other configurations (fig. $2.2 \mathrm{a}, \mathrm{b}, \mathrm{d}, \mathrm{e}$ ), we used a single node inside the box. For the chain configuration the size of the box was decreased in one dimension only, whereas for the 
cell model, the simple cubic and the FCC configuration the box was decreased in all three dimensions simultaneously. We calculate a free energy $F(d)$ of the node with the polymers, as a function of the distance $d$ between the nodes through the periodic boundary. The distance $d$ between the centres of the nodes (given in the number of lattice sites) is indicated in Figure 2.2. The free energy of interaction $\Delta F(d)$ is then specified by

$$
\Delta F(d)=F(d)-F(\infty)
$$

The reference value for the free energy of interaction $F(\infty)$ is the free energy when the nodes are so far apart that the polymers can only form loops.

The calculations are done in a canonical ensemble $(n, V, T)$, and thus with a fixed amount of polymers. Although we change the volume of the box one can imagine that there is an additional volume such that the total volume remains constant. As this volume only contains pure solvent for which the chemical potential is defined as 0 , it does not contribute to the free energy. The appropriate characteristic function is thus the Helmholtz energy. Here our interest is in the free energy for the system wherein the ends of all polymer chains are constrained to be on the node positions which are conveniently collected in the set of $n$ coordinates, $\left\{\mathbf{r}_{n}\right\}$, exactly specified by the input of the calculations.

Central in the SCF theory is a mean field free energy which is a functional of two complementary distributions, the volume fraction profile of the segments and a segment potential profile. This mean field free energy can be written in the generic form ${ }^{24}$ :

$F=U([\varphi])-\sum_{\mathbf{r}} \sum_{X} \varphi_{X}(\mathbf{r}) u_{X}(\mathbf{r})+\sum_{\mathbf{r}} \alpha(\mathbf{r})\left(\sum_{X} \varphi_{X}(\mathbf{r})-1\right)-\ln Q([u])$

In which $\varphi_{X}(\mathbf{r})$ is the volume fraction and $u_{X}(\mathbf{r})$ is the segment potential of segments type $X$ at position $\mathbf{r}$. We use the the Lagrange multiplier method, in the third term, $\sum_{\mathbf{r}} \alpha(\mathbf{r})\left(\sum_{X} \varphi_{X}(\mathbf{r})-1\right)$, to ensure that the sum of the volume fractions is one in every lattice site. The interaction energy $U([\varphi])$ between the segments depends on the volume fraction distribution. It is, for the Flory-Huggins equation of 
state, given by:

$$
U([\varphi])=\frac{1}{2} \sum_{\mathbf{r}} \sum_{X} \sum_{Y} \chi_{X Y} \varphi_{X}(\mathbf{r})\left\langle\varphi_{Y}(\mathbf{r})\right\rangle
$$

Here $\chi_{X Y}$ is the (Flory-Huggins) interaction energy between segments $X$ and $Y$ and $\left\langle\varphi_{Y}(\mathbf{r})\right\rangle$ is the average volume fraction of segment type $Y$ in the sites around $\mathbf{r}$ :

$$
\left\langle\varphi_{Y}(\mathbf{r})\right\rangle=\frac{1}{Z} \sum_{\mathbf{r}^{\prime}} \varphi_{Y}(\mathbf{r}) \delta_{\left|\mathbf{r}^{\prime}-\mathbf{r}\right|-1}
$$

where the Kronecker $\delta_{\left|r^{\prime}-r\right|-1}=1$ when $\left|\mathbf{r}^{\prime}-\mathbf{r}\right|-1=0$ and zero otherwise. $Z$ is the number of neighbours of the lattice site at $r$. The factor $\frac{1}{2}$ is introduced because each contact is counted double. Finally, $Q([u])$ is the partition function which depends on the segment potentials $u_{X}(\mathbf{r})$.

In the mean field Ansatz the partition function of the system can be rewritten in terms of single molecule partition functions $q_{i}$

$$
Q=\prod_{i} \frac{q_{i}^{n_{i}}}{n_{i} !}
$$

Wherein $n_{i}$ is the number of molecules of type $i$. The partition function can be evaluated efficiently by using the freely jointed chain model. Within this model $q_{i}$ is found by $q_{i}=\sum_{\mathbf{r}_{n}} G_{i}\left(\mathbf{r}_{n}, N+1 \mid\left\{\mathbf{r}_{n}\right\}, 0\right)$. In this case the summation can be limited to the sum over the set of coordinates $\mathbf{r}$ that lie within the nodes. Here $G_{i}\left(\mathbf{r}_{n}, N+1 \mid\left\{\mathbf{r}_{n}\right\}, 0\right)$ is called the end point distribution function and is effectively the sum of the statistical weights of all chain conformations ending at the node position $\mathbf{r}_{n}$ with segment $s=N+1$ while segment $s=0$ can be located within any node in the system.

All conformations that contribute to the end point distribution function at a location $\mathbf{r}$ will have the same Boltzmann weight for the last segment $s=N+1$. This term $G_{i}(\mathbf{r}, N+1)$, which is equal to $e^{-u(\mathbf{r}, N+1)}$ when $\mathbf{r} \in\left\{\mathbf{r}_{n}\right\}$ and zero otherwise, can thus be moved outside the summation. Since the chain must have come from one of $Z$ adjacent lattice 
sites, the remainder of the summation can be written as a summation of the end-point distribution function, of the chain without the last segment, on adjacent sites multiplied with the fraction of the paths $\frac{1}{Z}$ from adjacent site $\mathbf{r}^{\prime}$ that go from $\mathbf{r}^{\prime}$ to site $\mathbf{r}$.

$$
G_{i}\left(\mathbf{r}, N+1 \mid\left\{\mathbf{r}_{n}\right\}, 0\right)=G_{i}\left(\mathbf{r}_{n}, N+1\right) \frac{1}{Z} \sum_{\mathbf{r}^{\prime}} G_{i}\left(\mathbf{r}^{\prime}, N \mid\left\{\mathbf{r}_{n}\right\}, 0\right) \delta_{\mathbf{r}^{\prime}-\mathbf{r}}
$$

This equation is known as the propagator, because it relates walks that are $N+1$ steps long to one that is $N$ segments long. This process can be repeated, that is we can relate $G_{i}\left(\mathbf{r}, N \mid\left\{\mathbf{r}_{n}\right\}, 0\right)$ to a summation over $G_{i}\left(\mathbf{r}^{\prime}, N-1 \mid\left\{\mathbf{r}_{n}\right\}, 0\right)$, etcetera, until $G_{i}\left(\mathbf{r}, 0 \mid\left\{\mathbf{r}_{n}\right\}, 0\right)$ is reached. For this last one, we have $G_{i}\left(\mathbf{r}, 0 \mid\left\{\mathbf{r}_{n}\right\}, 0\right)=G_{i}\left(\mathbf{r}_{n}, 0\right) \equiv e^{-u\left(\mathbf{r}_{n}, 0\right)}$ for all nodepositions $\left(\mathbf{r} \in \mathbf{r}_{n}\right)$, while the end-point distribution $G_{i}\left(\mathbf{r}, 0 \mid\left\{\mathbf{r}_{n}\right\}, 0\right)=0$ for all remaining lattice sites $\mathbf{r} \notin\left\{\mathbf{r}_{n}\right\}$.

Hence, the partition function $q_{i}$ can be obtained by starting the calculation at $G\left(\mathbf{r}_{n}, 0 \mid\left\{\mathbf{r}_{n}\right\}, 0\right)$ and from these calculate $G_{i}\left(\mathbf{r}, 1 \mid\left\{\mathbf{r}_{n}\right\}, 0\right)$ and further on, via $G_{i}\left(\mathbf{r}_{n}, s \mid\left\{\mathbf{r}_{n}\right\}, 0\right)$, till $G_{i}\left(\mathbf{r}_{n}, N+1 \mid\left\{\mathbf{r}_{n}\right\}, 0\right)$ is reached, that is the propagators are executed in the reverse order as introduced above.

The end point distribution functions can further be used to determine the local volume fractions of the segments $\varphi_{i}\left(\mathbf{r}, s^{\prime}\right)$. More specifically, $\varphi_{i}\left(\mathbf{r}, s^{\prime}\right)$ is proportional to the end-point distribution functions that collect all statistical weight of those conformations that pass through coordinate $\mathbf{r}$ with segment $s=s^{\prime}$. It is the product of the two complementary propagators of the chain fragments, one leading from segment $s=0$ to segment $s=s^{\prime}$ and another one from segment $s=N+1$ to segment $s=s^{\prime}$.

$$
\begin{array}{r}
\varphi_{i}\left(\mathbf{r}, s^{\prime}\right)=C_{i} \frac{G_{i}\left(\mathbf{r}, s^{\prime} \mid\left\{\mathbf{r}_{n}\right\}, 0\right) G_{i}\left(\mathbf{r}, s^{\prime} \mid\left\{\mathbf{r}_{n}\right\}, N+1\right)}{G_{i}\left(\mathbf{r}, s^{\prime}\right)} \\
=C_{i} \frac{G_{i}\left(\mathbf{r}, s^{\prime} \mid\left\{\mathbf{r}_{n}\right\}, 0\right) G_{i}\left(\mathbf{r}, N-s^{\prime}+1 \mid\left\{\mathbf{r}_{n}\right\}, 0\right)}{G_{i}\left(\mathbf{r}, s^{\prime}\right)}
\end{array}
$$

Because the Boltzmann weight of segment $s=s^{\prime}$ is in both end point distribution functions we need to correct by division with $G_{i}\left(\mathbf{r}, s^{\prime}\right)$. In the second line we have rewritten the partition function. Because the 
polymer is symmetrical we only need to evaluate the propagator in one direction and can thus save computation time. The normalization constant $C_{i}$ is easily found as the ratio between the number of chains $n_{i}$ and the single chain partition function $q_{i}$.

$$
C_{i}=\frac{n_{i}}{q_{i}}
$$

The overall volume fraction distribution of polymers is found by a summation over the segments

$$
\varphi_{i}(\mathbf{r})=\sum_{s=0}^{s=N+1} \varphi_{i}(\mathbf{r}, s)
$$

The distribution of the monomeric solvent simply follows from the Boltzmann weight

$$
\varphi_{S}(\mathbf{r})=C_{S} G_{S}(\mathbf{r})=C_{S} e^{-u_{S}(\mathbf{r})}
$$

When the segment potentials are normalized to zero in the bulk, it is easily shown that $C_{S}=1$.

Now we still need a method to determine the segment potentials $u_{X}(\mathbf{r})$. Because the self-consistent solution we are looking for is the one with the lowest free energy. We need to optimize the free energy to the parameters $u_{X}(\mathbf{r}), \varphi_{X}(\mathbf{r})$ and $\alpha(\mathbf{r})$. This optimization then directly leads to the methods for determining the segment potentials from the segment volume fractions and vice versa. The optimization with respect to the volume fractions gives

$$
\frac{\partial F}{\partial \varphi_{X}(\mathbf{r})}=-u_{X}(\mathbf{r})+\sum_{Y} \chi_{X Y}\left\langle\varphi_{Y}(\mathbf{r})\right\rangle+\alpha(\mathbf{r})=0
$$

which specifies how to compute the segment potentials in the SCF machinery.

$$
u_{X}(\mathbf{r})=\sum_{Y} \chi_{X Y}\left\langle\varphi_{Y}(\mathbf{r})\right\rangle+\alpha(\mathbf{r})
$$

The optimization of the free energy with respect to the Lagrange parameter $\alpha(\mathbf{r})$ leads to the rule that we need to obey to the constraint 
$\sum_{X} \varphi_{X}(\mathbf{r})=1$. The optimization of the free energy with respect to the segment potentials gives the rule how to compute the volume fraction distribution from the potentials:

$$
\begin{gathered}
\frac{\partial F}{\partial u_{X}(\mathbf{r})}=-\frac{\partial \ln Q}{\partial u_{X}(\mathbf{r})}-\varphi_{X}(\mathbf{r})=0 \\
\varphi_{X}(\mathbf{r})=-\frac{\partial \ln Q}{\partial u_{X}(\mathbf{r})}
\end{gathered}
$$

This method for determining the volume fractions is however computationally less efficient than the previously described method. We have thus used eqn. 2.9 to calculate the volume fractions.

Now only the parameter $\alpha(\mathbf{r})$ remains to be defined. There is no clear way how to choose this alpha, but all possibilities should have in common that the value of $\alpha$ should increase when $\sum_{X} \varphi_{X}(\mathbf{r})>1$ and decrease when $\sum_{X} \varphi_{X}(\mathbf{r})<\mathbf{1}$. We choose to update alpha at each iteration step as:

$$
\alpha_{\text {new }}(r)=\alpha_{\text {old }}(r)+\eta\left(1-\frac{1}{\sum_{X} \varphi_{X}(\mathbf{r})}\right)
$$

where $\eta$ is a regularisation parameter which is taken small enough so that the equations do not diverge (typically $\eta=0.3$ gives a stable scheme. In any case, we do not terminate the iterations until a fixed point is reached for all $\alpha(\mathbf{r})$ values.

The above set of equations fully specifies how to compute the potentials from the volume fractions and vice versa. The numerical solution is routinely calculated with a Hessian-free minimization method. For the calculations in this chapter the L-BFGS method ${ }^{[57}$ was used. We obtained at least 7 significant digits for the potentials and for the volume fraction distributions. Using these we can evaluate the free energy we need in Equation 2.1, which is at the basis of the evaluation of the pair potentials.

Now that the free energy of the system is known we can determine an effective pair potential $\Delta F_{12}(d)$ for the different geometries. The effective pair potential is the pair potential that will reproduce the free 
energy of interaction $\Delta F(d)$ when all the interactions between pairs of nodes are summed together. We thus assume that the interactions are pair wise additive to determine $\Delta F_{12}(d)$. If this is indeed the case we should find the same $\Delta F_{12}(d)$ for all configurations. There are however many reasons why the interaction may not be pair wise additive. E.g. one node may be in between two other nodes blocking some of the bridging conformations between them. Therefore one of our targets is to quantify this loss in pair wise additivity.

$\Delta F_{12}(d)$ has been calculated for the four different particle configurations. The idea to find the pair potential for a given interaction geometry is the following; In a given interaction geometry it is trivial to find the number of neighbours at a given distance from the central node. Let us label these neighbours by $k_{1}=1, \cdots K_{1}$, where $K_{1}$ is the number of nearest neighbours. Similarly we number the next nearest neighbours as $k_{2}=1, \cdots, K_{2}$, etcetera. The distance to the central node for these neighbours is given by $d_{k_{1}}$ for the nearest neighbours and $d_{k_{2}}$ for the next nearest neighbour, etcetera. Then assuming that for this effective pair potential the pairwise additivity holds, we should recover the total interaction energy by summing the pair interaction over all the contributions of the neighbours:

$$
\Delta F(d)=\sum_{k_{1}=1}^{K_{1}}\left[\Delta F_{12}\left(d_{k_{1}}\right)\right]+\sum_{k_{2}=1}^{K_{2}}\left[\Delta F_{12}\left(d_{k_{2}}\right)\right]+\cdots
$$

We continue to account for the neighbours further away until they no longer contribute to the summation. The effective pair potential was calculated iteratively from the known $\Delta F(d)$ for a given interaction geometry. An initial guess for the effective pair potential $\Delta F_{12}(d)$ is made by dividing the total interaction potential by the number of nearest neighbours. Subsequently, the contribution from the neighbours further away is calculated based on this potential. These contributions are then subtracted from the total interaction potential. The remainder of the potential is then again divided by the number of nearest neighbours to get a new estimate of $\Delta F_{12}(d)$. This is repeated until $\Delta F_{12}(d)$ remains constant. Because the next nearest neighbours may lie at non-integer distances a cubic spline function was used to interpolate between the data points of $\Delta F_{12}(d)$. Obviously, for the IP geometry 
we find $\Delta F_{12}(d)=\Delta F(d)$ because there is just one neighbour involved.

For the cell model the effective pair potential cannot be calculated in this manner and we have simply divided $\Delta F(d)$ by twelve, because this is the number of nearest neighbours in an FCC lattice which is the most similar to the cell model.

\section{Results and Discussion}

We subdivide the results section into two parts. First we present the results for the one-gradient calculations, that is for the classical cell model. These calculations are inexpensive as they typically take less than a tenth of a second of CPU time for each distance. Most of these results are at least qualitatively known in the literature and are reproduced here for comparison. In the second part we will present results of the three-gradient calculations with a focus on the results for the isolated pair. These calculations take on the order of one minute of CPU time for each distance and determine the free energy with an error less than $0.01 k_{B} T$ within the assumptions of the model used here. At the end of this section we will focus on the pairwise additivity of the pair potential and show the results for the NoL, $\mathrm{cP}$ and FCC configurations.

\section{Cell Model Results}

In Figure 2.3 results for the radial density profiles, calculated with the cell model are presented in double logarithmic coordinates for the case $N=500, f=10$ and, $\chi=0.5$ and $\chi=0.0$. In the central region $2<r<10$ where the curvature is important, the polymer density decays like a power law. The exponents are very close to those for star polymers which are $-4 / 3$ in a good solvent and -1 in a theta solvent. $\frac{58}{6}$ The profiles found here therefore look very similar to those reported by Wijmans et al. ${ }^{59}$ for star polymers. The region for which the power law behaviour is observed is small as the polymers are relatively short. For $\chi=0.5$ the edge of the polymer brush is reached at $r \approx 15$ where the polymer density starts to decrease exponentially. As the thickness of the corona layer in good solvent is larger than that of the theta solvent, the profiles cross each other around $r=20$. The profile for the good solvent hits the upper boundary at $r=30$ and crosses this boundary 


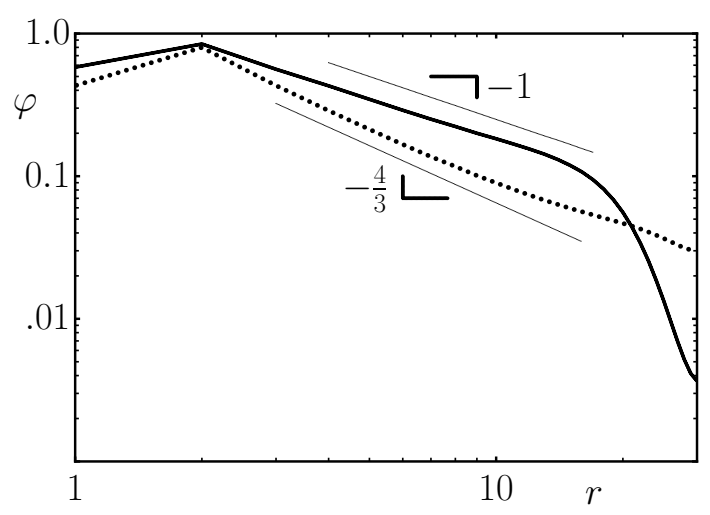

Figure 2.3: Radial polymer volume fraction profile around a node in the cell model. $N=500, f=10$. The continuous line is for theta solvent $\chi=0.5$ whereas the dotted line is for good solvent, $\chi=0$. The slope of the power-law region of the profile is indicated, which is -1 for theta solvent and $-\frac{4}{3}$ for good solvent.

with zero slope (this is imposed by the boundary condition). The finite concentration at the boundary implies that bridges are formed. In the theta-solvent $\chi=0.5$ the reflecting boundary is still far from the edge of the polymer brush and a negligible number of bridges is present. For $\chi=0$ and $r>15$ the polymer density is higher than expected from the power law behaviour. This is due to polymer loop conformations that in a free micelle would extend beyond the edge of the cell are now folded back into the cell.

The dimensionless free energy of interaction $\Delta F(d)$ is computed in the cell model for different values of the cell size $M_{r}$. In Figure 2.4 we give a summary of the interaction free energy curves for the cell model. The default system has chains with a length of 50 segments, $N=50$, five chains per node, $f=5$, and a good solvent, $\chi=0$.

In Figure 2.4 the effect of the variation in the number of polymers per node is presented, in Figure 2.4 $\mathrm{b}$ we show the result for the variation of the chain length and finally in Figure 2.4 the solvent quality was varied. At large distances, $d \rightarrow \infty$, the free energy of interaction 

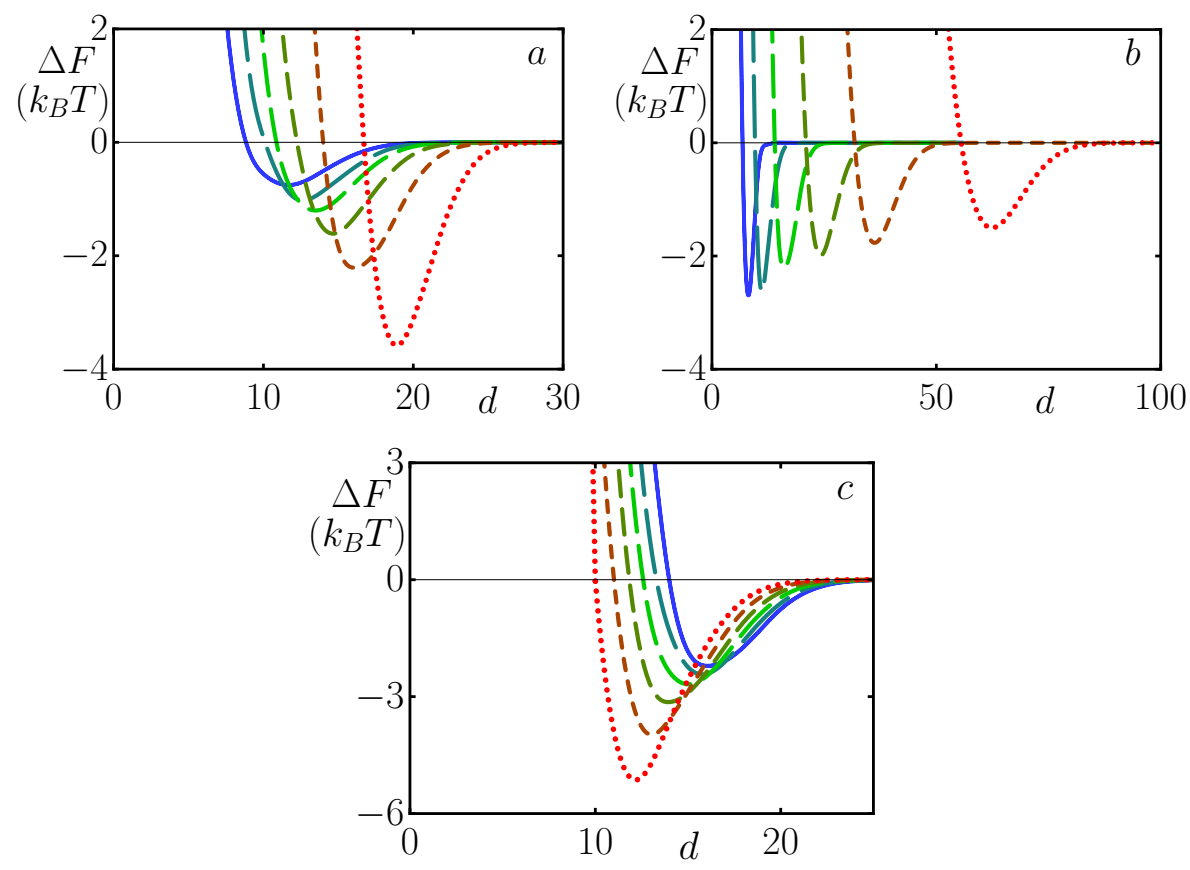

Figure 2.4: Free energy of interaction $\Delta F$ for the cell model as a function of the distance $d=2 * M_{r}$ between the node and its mirror image. a) $N=50, \chi=0$ and the number of polymers per node ranging from left to right : $f=1(-), 1.5,2,3,5,10(\cdots)$ b) $\chi=0, f=5$, and the chain length varied from left to right: $N=12(-), 25,50,100$, 200, $500(\because)$. c) $N=50, f=5$, and varied solvent quality: from left to right: $\chi=0.5$ (theta solvent," " "), 0.4, 0.3, 0.2, 0.1, 0 (good solvent,-).

is zero. As soon as the coronas 'touch' each other, bridges form and an attraction is found. Hence, $\Delta F$ becomes negative. At small values of $d$ the corona layers are strongly compressed and steric repulsion is found. The interaction curve can be characterized by, the depth, width and position of the attractive well. Although the steepness of the repulsion also varies, it is usually so steep that the range over which the micelle behaves as a soft particle rather than a hard sphere is small and the excluded volume thus does not change much due to the varying steep- 
ness of the repulsion. Because there can only be an integer number of layers in the cell model, the free energy of interaction is only available at even values of $d$. This is why the curves appear somewhat kinky. In reality the interaction curves should of course be smooth.

In Figure 2.4 the dependence of the free energy of interaction on the number of chains per node, $f$, is presented. The depth of the attractive well increases and both the minimum and the onset of the steric repulsion occur at greater distances $d$ with an increasing $f$. Both trends are easily explained. The attraction is due to the transformation of loops into bridges and the more bridges can form the deeper the minimum. The depth of the minimum however does not scale linearly with the number of arms but roughly with $\Delta F_{\min } \sim f^{0.65}$. Due to the limited number of points, the depth of the well is not determined accurately enough to conclude that there is power law behaviour.

A larger number of chains on a node also increases the height of the corona and the micelles will thus attract each other at greater distances. An increase in the number of polymers per node however also leads to an increased polymer density in the corona and thus an earlier onset of the steric repulsion. Therefore the minimum shifts to larger separations $d$. Fitting of the position of the minimum as a function of the number of arms gives to a good approximation $d_{\text {min }} \propto f^{0.2}$, which is expected from the Daoud Cotton model. ${ }^{58}$ Below in Figure 2.9 we elaborate more on the depth of the interaction curve as a function of $f$.

The chain length dependence for the free energy of interaction is presented in Figure 2.4 $\mathrm{b}$. The position of the minimum is found to scale with the corona thickness which in turn depends on the length of the polymers. In the limit of large $N$ values $d_{\min } \propto N^{3 / 5}$ is found to a good approximation, which is in accordance with the Daoud Cotton model. ${ }^{58}$ The depth of the interaction minimum is, on the other hand, a weak function of the chain length and it decreases with increasing chain length. We further observe a broadening of the well. We will return to this point in Figure 2.10.

The third variable that is relevant to investigate is the solvent quality. As can be seen in Figure 2.4c the relevant features of the free energy of interaction systematically shifts to larger $d$ values when the solvent quality improves. As mentioned before, the minimum is generated by steric repulsion at short distances and attraction at larger distances due 
to the loop to bridge transitions. At large distances the attraction is not much affected by the solvent quality. The main effect is the onset of the repulsion which occurs at larger distances when the solvent quality is better. As illustrated by the radial volume fraction profiles in fig. 2.3, the corona is more swollen in a good solvent. This swelling of the corona is due to the increased value of the segment virial coefficient $v=1-2 \chi$, which specifies the repulsion between segments that encounter each other in the corona. The overlap between the coronas is more repulsive the larger the virial coefficient is. The position at which the repulsion is larger than the attraction will thus shift to greater distances $d$. The width and depth of the well will thus decrease and the minimum of the interaction curve shifts outward.

\section{Three-Gradient Models}
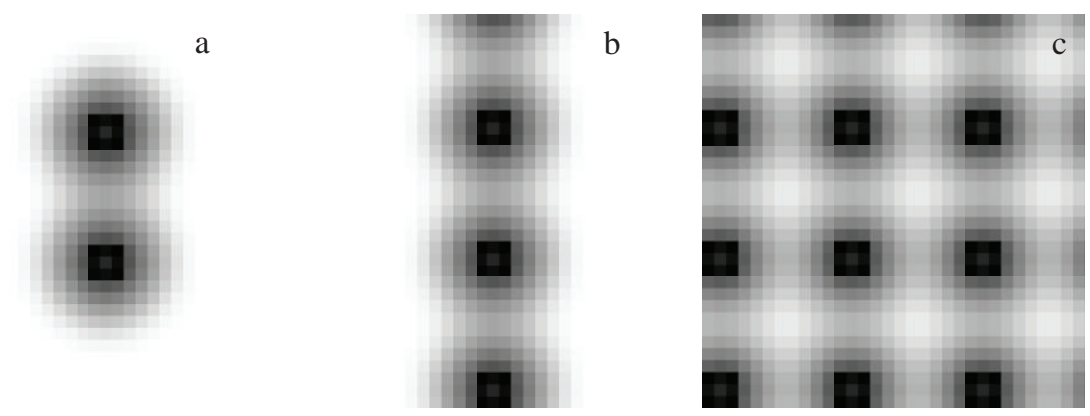

Figure 2.5: A cross section through a three-gradient density profile of the polymer segments. a) an isolated pair (IP), b) a line of nodes (NoL), c) a simple cubic $(\mathrm{cP})$ arrangement of nodes. The cross section is taken through the centres of the nodes. $N=50 \chi=0.5, f=5$. The dark areas indicate a high polymer density.

It is illustrative to first discuss some typical examples of the polymer density as found in the three-gradient calculations. Figure 2.5 shows a polymer density plot for a cross section taken such that the plane crosses the nodes through their centres, for a) the isolated pair (IP), b) a series 
of nodes on a line and $\mathrm{c}$ ) the $\mathrm{cP}$ arrangement of nodes. In the cross sections the square lattice is clearly visible. A higher polymer density is indicated by a darker colour. The polymer density for the IP (Figure 2.5 ) shows that the polymer is arranged slightly asymmetrically around the two nodes. Because bridges can be formed, there are more polymer conformations possible between the nodes and the polymer density is thus higher. Similar effects are seen for the string of nodes on a line (Figure 2.5p). Halfway in between the nodes, the polymer density is a bit higher than on comparable distances in the other directions. Also in the simple cubic arrangements (Figure 2.5k) we see an inhomogeneous distribution around the node. In the contact regions the polymer density is a bit higher due to the bridging that takes place.
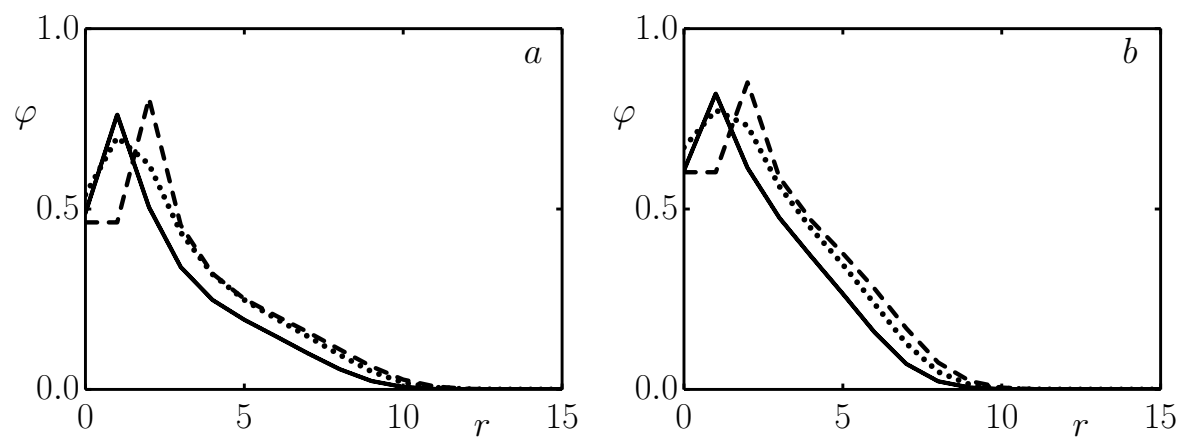

Figure 2.6: The volume fraction of the polymer as a function the distance $r$ in lattice sites from the centre of the node for $\chi=0$ (a) and $\chi=0.5(\mathrm{~b}) . \quad f=10, N=50$. FCC lattice $(-)$, cell model (- - -), simple cubic $(\cdots)$.

Just as in the cell model, we can determine the volume fraction, as a function of the distance from the centre of an isolated node, for the three-gradient calculations. In Figure 2.6 radial profiles are shown for micelles, with $N=50$ and $f=10$, in the cell model, on a cubic lattice and on an FCC lattice, for both good $\chi=0$ (panel a) and theta $\chi=0.5$ (panel b) solvents. The curve for the $\mathrm{cP}$ and the FCC lattice was taken through the centre of one of the faces of the cube/parallelepiped. Because the density at the corners of the cube shaped node was higher than in the middle of the faces, the amount of polymer that would be 
found if one integrated over the polymer density is a bit lower for the cP lattice than for the cell model. Because the distance between the centres of the nodes was kept the same for the $\mathrm{cP}$ and FCC lattice, the volume of a lattice site in the FCC lattice is smaller than in the cubic lattice. As a consequence there are more lattice sites within a certain radius of the centre of the node in the $\mathrm{FCC}$ lattice than in the $\mathrm{cP}$ lattice and the polymer density for the FCC lattice will thus be lower than that of the $\mathrm{cP}$ lattice.

As the middle blocks try to move outward, to reduce the steric repulsion between them, the end-groups are pulled to the surface of the node. This increases the polymer concentration at the surface and lowers the polymer concentration in the centre of the node, as seen in Figure 2.6. As expected, the profiles in Figure 2.6 are very similar. This indicates that the cell model is giving a reasonable prediction for the typical distribution of segments around a node. As the details of the radial profiles were already discussed for the cell model, see fig. 2.3, we will not repeat them here. The polymers are too short to be able to clearly see power law behaviour in fig. 2.6.

When two nodes are sufficiently close to each other, bridges may form. It is of interest to quantify the number of bridges, which can be computed similarly as for the evaluation of bridges between two surfaces. 50 The number of bridges is given by the total number of chains minus the number of loops. The latter can simply be computed from evaluating the number of chains that start and end at the same node. This is done by recalculating the single chain partition function $q_{i}$, with just one of the nodes present and without adjusting the segment potentials. With $n_{i}=C_{i} q_{i}$ and the $C_{i}$ from the calculation with all nodes present, the number of polymers in a loop configuration on that node is determined.

The evaluation of the number of bridges in the cell model is problematic as it is not clear whether a loop or bridge is formed when the polymer crosses the periodic boundary more than once. For a pair of isolated nodes the mentioned procedure is easily implemented which leads to the number of bridges for a given position of the two nodes. Throughout this chapter the nodes are oriented along either the $\mathrm{x}$ or $\mathrm{y}$ or z-direction in the lattice, so that the distance between the nodes is an integer number of lattice sites. However, the nodes may also be ori- 


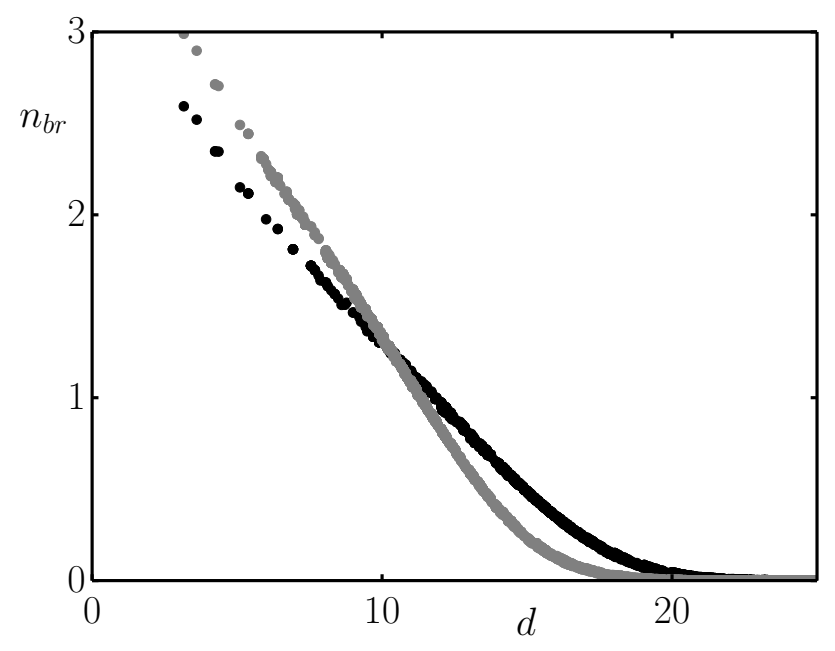

Figure 2.7: The number of bridges $n_{\mathrm{br}}$ between a pair of isolated nodes, on a cubic lattice for $\chi=0$ to $\chi=0.5$, as a function of the distance between the centres of the nodes $d$. The black dots belong to $\chi=0$ and the grey dots belong to $\chi=0.5 ; f=5$ and $N=50$.

ented differently with respect to the lattice directions. Information on the number of bridges as a function of the distance between the nodes for arbitrary orientation of the nodes in the lattice can give information on the presence or absence of a lattice artefact in our calculations. In short, we have generated a large number of random positions for the pair of nodes in our system and for each of these positions we have evaluated the number of bridges $n_{\mathrm{br}}$. In Figure 2.7 the number of bridges between an isolated pair of nodes $n_{\mathrm{br}}$ is shown for the default conditions for both $\chi=0$ and $\chi=0.5$. The number of bridges scales with the amount of the polymer loops at the mirror plane between the micelles (not shown). For each point where a polymer in a loop conformation crosses this mirror plane, the mirror image of the second part of the conformation forms a new bridging conformation. As the number of polymers that cross the mirror plane decreases almost linearly with increasing distance between the nodes, the number of bridges also decreases linearly with increasing distance $d$. This is especially clear for $\chi=0.5$. Even though the points in Figure 2.7 do not lie perfectly on a 
line the deviations are small, proving that the orientation of the nodes relative to the grid has little influence on the number of bridges.

We can evaluate the number of bridges also in more complicated configurations of the nodes, however, these calculations may become involved as soon as more particles are involved (NoL, cP or FCC), because we should distinguish bridges between nearest neighbours from those between next nearest neighbour and so forth. As the comparison with the cell model is not straightforward, we will not further analyse the number of bridges and rather focus on the free energy of interaction, which is the result of the bridging attraction and steric repulsion.

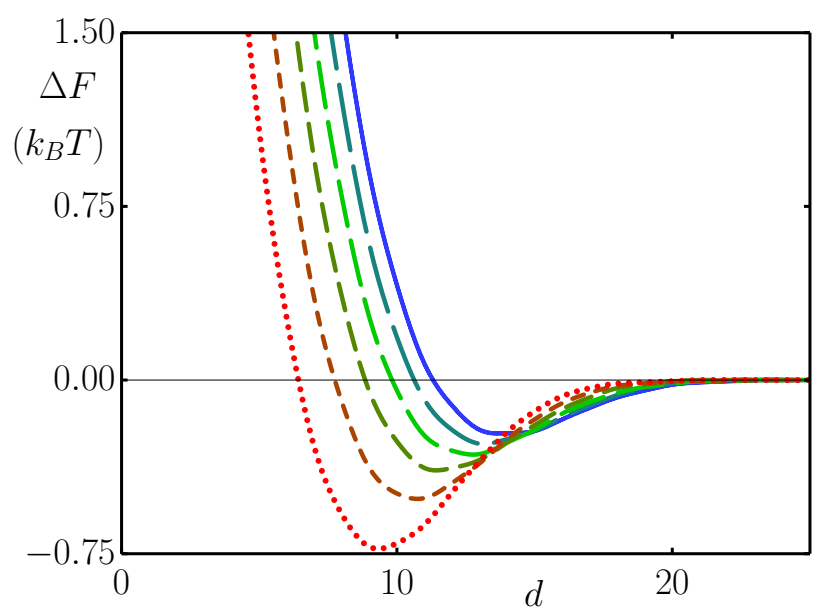

Figure 2.8: The interaction free energy per node $\Delta F$ between a pair of isolated nodes on a cubic lattice for $\chi$ ranging from 0 to 0.5 in steps of 0.1 . The blue continuous line belongs to $\chi=0$ and the red dotted line to $\chi=0.5 ; f=5$ and $N=50$.

We have used the simplest particle configuration for which bridge formation occurs, the isolated pair(IP) on a cubic lattice, to study how the free energy of interaction depends on the length of the polymers $N$, the number of polymers per node $f$ and the Flory interaction parameter $\chi$. The interaction curves, for different values of the solvent quality $\chi$, are shown in Figure 2.8. In this case there were 10 polymer chains, 5 per node $(f=5)$ and each polymer had $N=50$ segments. With 
decreasing distance, the loop to bridge attraction is expected to increase as there are more bridging conformations (see fig. 2.7). The steric repulsion however also increases with decreasing distance because the corona layers are compressed. The resulting well depth is therefore an interplay with the strengths and the ranges of these two contributions. For a good solvent the corona layer is more extended and the repulsive contribution becomes longer ranged which causes the depth of the well to become less deep with increasing solvent quality.

Qualitatively the interaction curves are similar to those of the cell model. With increasing values of $\chi$, the trend of increasing well depth and a decreasing distance at which the minimum of the interaction is observed, is clearly visible and the results qualitatively compare well to the result of the cell model discussed above in fig. 2.4k. Quantitative differences are obvious. Most importantly the absolute value for the free energy of interaction $\Delta F$ is much smaller for the IP case. This is easily explained, for the IP there is just one direction in which a bridge can be formed whereas for the cell model a bridge can be formed in all directions. As shown in fig 2.5 the chains remain roughly isotropically distributed around the node when the other node is in its vicinity. As a result only a few chains can form bridges and the free energy of interaction remains modest. Another quantitative difference is the observation that the interaction curves are shifted to shorter distances for the IP as compared to the cell model and the steric repulsion is less steep. This is due to the fact that for the IP the chains have room to move out of the gap between the nodes when the nodes are forced towards each other. This is not possible in the cell model, the $\mathrm{cP}$ and the FCC cases.

Next, we choose to focus on the depth of the minimum in the free energy of interaction curve. We use the notation $\Delta F_{\min }$ and note that the negative value of this quantity is kept to remind ourselves that there is an attractive well.

For the IP interaction geometry we collected $-\Delta F_{\min }$ as a function of the number of chains per node $f$. In Figure 2.9 this dependence is shown in double logarithmic coordinates. Even though the range over which we can change $f$ is limited, it is found that the depth of the attractive well increases with $f$ as a power law. The exponents found range from 0.44 for $\chi=0$ to 0.51 for $\chi=0.5$. Semenov et al. did predict power law behaviour for this dependence, but the expected coefficients 


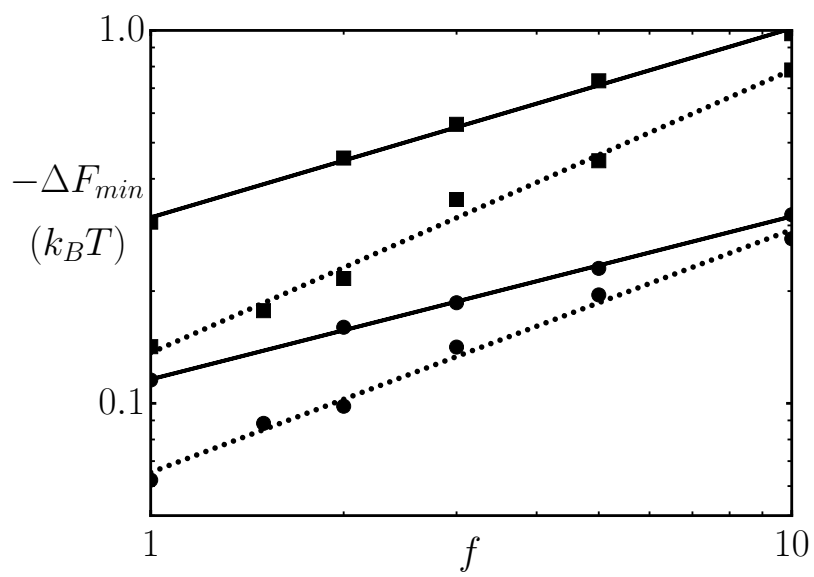

Figure 2.9: The depth of the attractive well $\Delta F_{\min }$, as a function of the number of chains per node $f$, on a double logarithmic scale, for the cell model $(\cdots)$ and the isolated pair $(-)$ for $\chi=0$ (Spheres) and $\chi=0.5$ (Squares). For the the cell model $\frac{1}{12} \Delta F_{\min }$ is plotted. The lines are the power law fits. $N=50$

ranged from 0.3 to 0.33 for $\chi=0$ to $0.5 . \stackrel{43}{ }$ Possibly, the relatively short chain length, $N=50$, has influenced the coefficients. These results can be compared to the cell model results presented in fig. 2.4a. Also for the cell model power law dependence is found and the results are presented in fig. $2.9 \mathrm{~s}$ dotted lines. In this case the free energy of interaction was divided by the expected surrounding of 12 , which corresponds to a FCC surrounding. For the cell model the power law coefficients ranged from 0.65 for $\chi=0$ to 0.76 for $\chi=0.5$ which is significantly larger than for the IP geometry. The larger coefficient for the cell model is probably due to the reduced steric hindrance between the bridge forming chains compared to the IP geometry. For the IP geometry there is only one way to form bridges and if multiple bridges are formed there will be steric hindrance between them. In the cell model bridges can however be formed in any direction and there is thus no strong steric repulsion between bridge forming chains.

The effect of the polymer chain length on the depth of the attractive well, for the IP and the cell model, is shown in Figure 2.10. (For the 


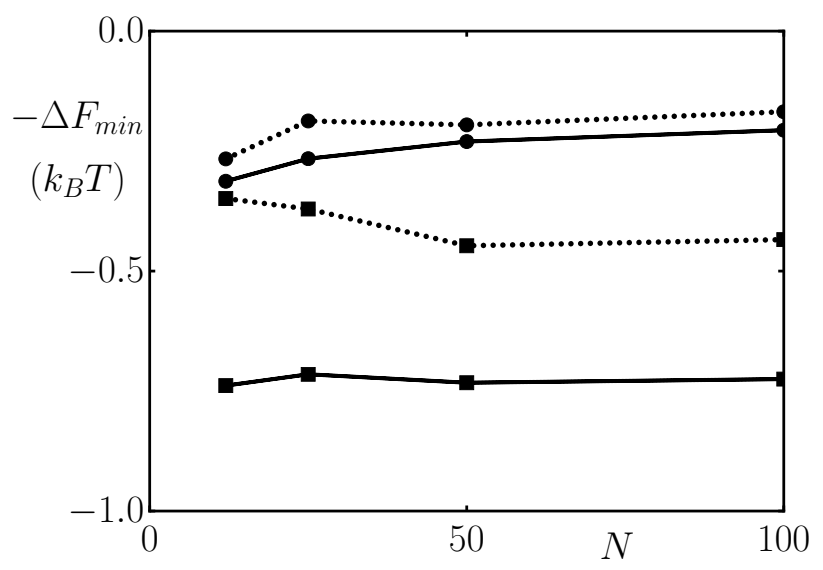

Figure 2.10: The depth of the attractive well $\Delta F_{\min }$ as a function of the chain length for a good solvent $\chi=0$ (spheres), and a theta solvent $\chi=0.5$ (squares) both for the cell model $(\cdots)$ for which $\frac{1}{12} \Delta F_{\min }$ is given as well as for the IP geometry $(-) ; f=5$.

cell model the free energy of interaction was again divided by 12). For the IP there is hardly a chain length dependence at $\chi=0.5$, for $\chi=0.3$ (not shown) and $\chi=0$ there is a weak decrease of the well depth with $N$, which seems to level off at large values of $N$. The weak chain length dependence is unexpected as the total number of bridges that can form is given by $f$ and this quantity is fixed. For the cell model the distance to a mirror image of the node could only be changed by 2 lattice sites at a time. This reduces the accuracy with which the minimum can be determined, especially for the chains of 12 and 25 segments. It is thus not clear whether the decrease of the well depth with increasing length in a theta solvent is significant for the cell model, although the well depth seems to decrease by about $10 \%$ going from $N=50$ to $N=500$. For a good solvent the cell model shows the same trend as the IP, as can be seen in Figure $2.4 \mathrm{~b}$.

Let us now compare the results for the free energy of interaction between the nodes, for the cell model, the IP, the NoL, the cP and the FCC configurations. In Figure 2.11 the interaction energy per node is presented for these interaction geometries with $f=5$ and $N=50$, 

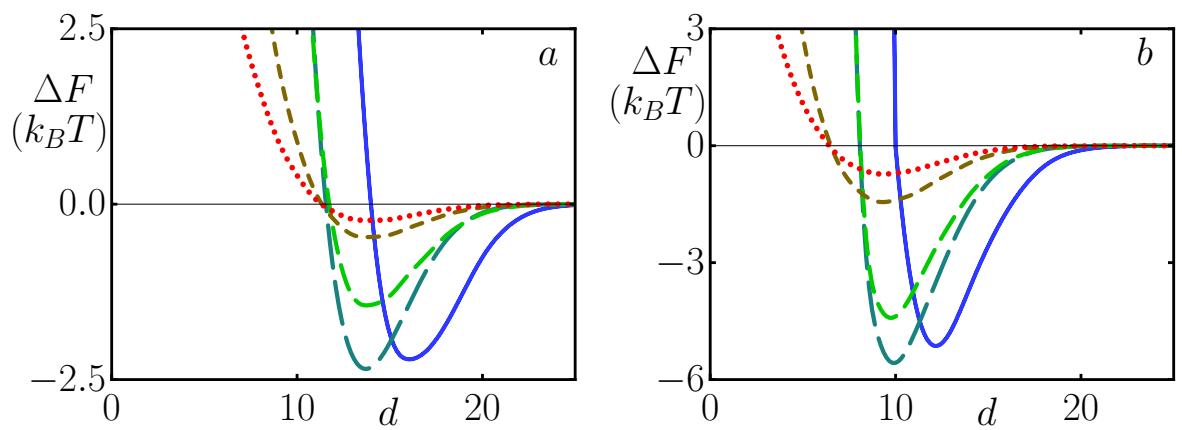

Figure 2.11: The free energy of interaction per node as a function of the distance $d$ between nodes measured in lattice sites, for $f=5$ and $N=50$. a) $\chi=0$ (b) $\chi=0.5$. Results are collected for different interaction modes. From the red dotted line to the continuous blue line: IP, NoL, cP, FCC and the cell model.

for a good solvent (panel a) and a theta solvent (panel b). In these calculations we made no corrections for the number of neighbours per node. Therefore the absolute value of the free energy of interaction differs a lot between the configurations. Below we will present the corresponding 'effective pair potentials' which correct for the number of neighbours. For the cell model the attractive well is shifted to greater distances. This is because the number of neighbouring nodes increases with the square of the distance, whereas the number of nodes remains constant for all other particle configurations. This gives an extra $\log d^{2}$ term to the attraction, resulting in an increased long range attraction for the cell model.

At a smaller cell size a neighbouring node is seen in all directions through the reflecting boundary conditions, there is thus a strong steric repulsion between the node and its mirror images when the cell size is reduced. Hence, the onset of the repulsive part of the interaction occurs at greater distances as well. The minima are at a shorter range for $\chi=0.5$ than for $\chi=0.0$, which is expected from the reduced swelling at $\chi=0.5$.

For the IP, the NoL and $\mathrm{cP}$ interaction geometries, the depth of the attractive well is proportional to the number of nearest neighbours. 

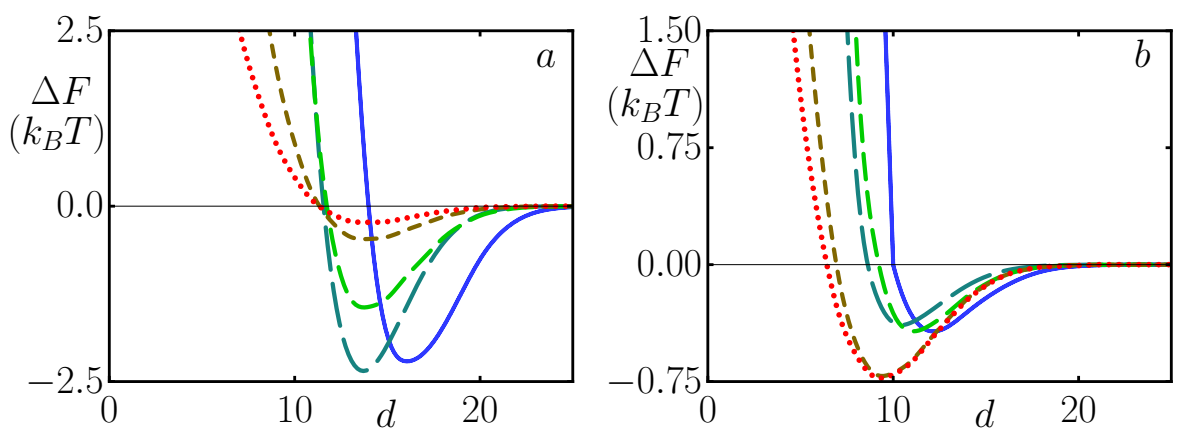

Figure 2.12: The effective pair potentials $\Delta F_{12}$ in units of $k_{B} T$ as a function of the distance $d$ in lattice sites, as explained in the text, for $\chi=0$ (a) and $\chi=0.5(\mathrm{~b})$. The free energies of interactions as presented in fig. 2.11 have been used for the evaluation of the pair potentials. Again $f=5$ and $N=50$. From the red dotted line to the continuous blue line: IP, NoL, cP, FCC and the cell model.

For the FCC configuration the depth of the attractive well is less than the depth expected from the number of nearest neighbours. About $\frac{5}{6}$ of the expected value for $\chi=0$ and $\frac{2}{3}$ for $\chi=0.5$. For the simple cubic configuration a deeper attractive well would be expected as well, because there are still twelve next nearest neighbours which are near enough to form a bridge. Assuming the interactions are the same as for an isolated pair, the sum of all these interactions should give a potential well deeper than the one found here for the cubic and the FCC configuration.

In Figure 2.12 we show the effective pair potentials $\Delta F_{12}(d)$ for the different interaction geometries, again for the two solvent qualities: good solvent, panel (a) and theta solvent panel (b). These pair potentials were extracted from the interaction free energy curves presented in Figure 2.11. To compare these effective pair potentials with the free energy per node from the cell model, the free energy in the cell model was divided by 12 assuming that there are twelve nearest neighbours as in an FCC or a hexagonal configuration. The same approach to obtain a pair potential from the cell model was used by Sprakel et al. 44

As can be seen in Figure 2.12, the attractive part of the effective pair 
interaction is practically the same for the IP and the NoL configurations, especially for the good solvent case for which the curves overlap. The repulsion between the nodes in the NoL configuration increases more rapidly for $\chi=0.5$, compared to the IP, because there is less space for the chains to escape to in the NoL configuration. There is a large difference between the IP and the configurations where the node is surrounded by other nodes in all directions, the cell model, the $\mathrm{cP}$, and the FCC configuration, especially for $\chi=0.5$. The repulsion occurs at larger distances and the potential well is less deep than for the IP. This increase of the distance at which the repulsion occurs could be expected. As the nodes come closer to each other the volume per node becomes smaller until there is not enough space left to fit the polymers. At short distances the interaction potential should thus go to infinity for the cell model, the $\mathrm{cP}$ and the FCC configuration. For the NoL and the IP there is still space for the polymers to escape to when the nodes touch each other and the interaction potential grows only modestly.

The well depth for the cell model, cP, and FCC configurations is smaller than for the IP. This is at least partially caused by the delayed onset of the repulsion in the IP. An additional reason is that one node can block bridge conformations between two adjacent nodes reducing the strength of the attraction between those nodes, which is important for bridges made with next nearest neighbours. One would also expect that each additional neighbouring node will decrease the free energy less than the previous one, because fewer chains are remaining for the bridges to form (some are already engaged in bridging). A different way to view this is by considering the entropy due to the number of polymer conformations. With each additional neighbour the number of possible polymer conformations is increased by some amount. Because the free energy scales with the natural logarithm of the number of conformations, the free energy is expected to decrease less with each additional neighbouring node. The average attraction between the nodes will therefore be lower than for an isolated pair.

The difference between the cell model, $\mathrm{cP}$ and FCC configurations is smaller. The depth of the attractive well is almost the same for these three configurations. The curve for the FCC configuration is shifted to a bit smaller distances than that of the $\mathrm{cP}$ configuration. This may be because the number of lattice sites that can be reached in a certain 
number of steps from the node is bigger for the FCC lattice than for a cubic lattice. The volume can thus be used more efficiently with an FCC lattice.

The pair potential obtained from the cell model is thus not such a bad approximation as long as one node is surrounded by many neighbours, that is when the overall node concentration is relatively high. At lower node concentrations, the micelles in the gel will likely form a more porous, open structure wherein each node is no longer surrounded by other nodes on all sides. In this case the cell model will underestimate the attraction and will thus not be able to predict the properties of the gel, such as the critical point for phase separation, correctly.

Based on the interaction free energy given in Figure 2.12 the second virial coefficient, $B_{2}$, can be calculated with Equation 2.17. With these $B_{2}$ values it is possible to estimate whether the flower-like micelles can phase separate into a dilute and a concentrated gel phase. Vliegenthart et al. reported the critical $B_{2}$ values for several forms of the potential. The critical $B_{2}$ values ranged from -8.9 to -5.5 times the volume of the repulsive core of the particles. $\underline{60}$ In the same way as Vliegenthart et al., we define a distance $d^{\prime}$ at which $\Delta F(d)=0$ for the first time. Based on this distance we calculate the volume of the repulsive core $V_{\text {core }}$ of the micelles with Equation 2.18. Subsequently we can calculate a normalised $B_{2 N}$ by dividing $B_{2}$ by $V_{\text {core }}$ as in Equation 2.19 .

$$
\begin{array}{r}
B_{2}=\frac{1}{2} \int 4 \pi d^{2}\left(1-e^{-\Delta F(d)_{12}}\right) \mathrm{d} d \\
V_{\text {core }}=\frac{\pi}{6} d^{\prime 3} \\
B_{2 N}=\frac{B_{2}}{V_{\text {core }}}
\end{array}
$$

Using this $B_{2 N}$ we find that for the IP, the boundary for phase separation is the line from $\chi=0.2$ and $f=1$ to $\chi=0.5$ and $f=10$. For combinations with a lower $\chi$ and a larger $f$ the steric repulsion is strong enough to prevent phase separation. This dependence on the number of polymers per node is opposite to what would be expected and what is found experimentally. ${ }^{45}$ As $f$ increases one would expect the number of bridges to increase and thus an increased attraction. This illustrates 
that the pair potential as found from the IP is not suitable to describe the aggregation of more than two micelles. Alternatively, when the effective pair potential from the $\mathrm{cP}$ or FCC configuration is used, no phase coexistence is predicted, even for $\chi=0.5$ and $f=10$. These results are also in disagreement with the findings of Filali et. al. who observed phase separation for $f>6$ for PEO polymers with hydrophobic ends in micelles swollen with oil, although their core was larger relative to the size of the corona. $\frac{45}{4}$ The pair potentials found from the $\mathrm{cP}$ or FCC configurations thus underestimate the average attraction between the nodes near the critical point.

The SF-SCF calculations show that the interactions between the nodes are not pairwise additive, clearly exemplified by the large difference between the effective pair potentials of the IP or NoL interaction geometries and the more isotropic interaction geometries. When the pair potentials are used in coarse-grained computer simulations, one should take this into account. Especially when the density around the node is not radially isotropic, as expected near a critical point, where fluctuations in density are large, or near an interface between the gel and a dilute solution, artefacts can be expected from having just one pair potential (e.g. tuned for the homogeneous surroundings). Because the attraction is weaker when a node is surrounded by more neighbours, it should be relatively easy to remove some of the neighbouring nodes. The surface tension of such a gel will therefore be relatively low and pores can easily be created. This allows particles and perhaps even other polymer networks to penetrate the gel.

\section{Conclusion}

We have performed one- and three-gradient SF-SCF calculations to determine the pair potential between nodes in a network of telechelics for different configurations of the nodes. We used a simple model wherein the two ends of the chain are constrained in predefined nodes and the intermediate segments of the chain are in good or theta solvent.

At small distances between the nodes, the coronas of the flower-like micelles overlap resulting in steric repulsion. At larger distances the increased entropy due to bridge formation gives attraction. Hence a 
curve with a local minimum was observed.

With the cell model we found that the position of the minimum scales with the length of the chain $N$ roughly as $N^{\frac{3}{5}}$ and as $f^{\frac{1}{5}}$ with the number of chains per node, as expected from the Daoud-Cotton model. ${ }^{58}$ With increasing $\chi$ the position of the minimum moved closer to the node and the attraction became 2 to 3 times stronger. The depth of the attractive well also increased with an increasing number of polymers per node. This increase decreased with each additional polymer chain. Roughly scaling as $f^{0.65}$ for $\chi=0$ and $f^{0.75}$ for $\chi=0.5$.

For the isolated pair on a simple cubic lattice the same trends were found as for the cell model. Except that the depth of the well scaled with the number of polymers per node as $f^{0.44}$ for $\chi=0$ and $f^{0.51}$ for $\chi=0.5$. For both the cell model and the isolated pair there was also a weak trend of decreasing well depth with increasing polymer length at $\chi=0$.

For the $\mathrm{cP}$ and FCC configuration and the cell model, the depth of the attractive well in the effective pair potential was about $60 \%$ of that for the isolated pair(IP) and the nodes on a line (NoL) at $\chi=0.5$. For the FCC and $\mathrm{cP}$ configuration the attractive well was shifted outward compared to the IP and NoL configuration, due to the increased steric repulsion. The well of the cell model lies even further out because the volume per node, for a given inter node distance, is the smallest in the cell model and the steric repulsion is thus the strongest. At the same time the attraction has a longer range, as the number of possible end points increases as the cell model becomes bigger. For $\chi=0$ the depth of the attractive well for the $\mathrm{cP}$ and FCC configuration and cell model is about $85 \%$ of that of the isolated pair.

In most experimental systems $\chi$ is close to 0.5 . The strength of the interaction than varies considerably with the number of neighbours the interacting nodes have. This is nicely illustrated when one tries to predict the phase behaviour based on the different pair potentials. Based on the pair potential of the isolated Pair, phase separation should occur over a wide range of $f$ and $\chi$ values, whereas based on the potential from the $\mathrm{cP}$ or FCC configuration no phase separation will occur within the range of $f$ studied here. Although, following the trend, phase separation is expected to occur at $f \approx 13.5$, which is in turn higher than the experimentally observed critical $f \approx 6 . \underline{45}$ It is thus important to adjust 
the interaction potentials based on the average number of neighbours the nodes are expected to have. Ideally, one would use a non-pairwise additive potential to calculate these interactions.

Overall the pattern of interaction is the same for the cell model and the other $3 \mathrm{D}$ configurations. The interaction however does occur at greater distances for the cell model because the number of nodes seems to increase as the distance between them becomes bigger. The depth of the attractive well is the roughly the same for the cell model and the other densely packed configurations but considerably less deep than that of the isolated pair and chain configurations. Using the cell model to determine the pair interactions for a course-grained model will therefore lead to an overestimate of the repulsion at high concentrations and underestimate the attraction at lower concentrations. We can thus conclude that using the potentials of the cell model will underestimate the net attraction between the nodes.

To further study gels with telechelic polymers we did hybrid Monte Carlo SF-SCF simulations, in which the nodes are moved by a Monte Carlo scheme and the SCF equations are solved for each snapshot. In such a simulation the pair potential does not need to be imposed as in particle based MC simulations. These simulations are described in chapter 3 and 4

Another avenue of future research is the study of micelles made of ABC polymers. As all the polymers need to form bridges it is expected that the two types of $\mathrm{ABC}$ micelles will attract each other much stronger than ABA micelles. Such networks would be stiffer and stronger than ABA networks while retaining their self healing properties. 


\section{CHAPTER 3}

\section{Hybrid Monte Carlo Self-Consistent Field Approach to Model a Thin Layer of a Polyelectrolyte Gel near an Adsorbing Surface}

Thin layers of surface bound (polyelectrolyte) hydrogels are used for measuring the concentration of metal ions in electrolyte solutions. The gels are composed of polymers with conformational degrees of freedom on the nanometer scale. The polymer conformations are affected by the presence of physical crosslinks in the gel on a five to ten times larger length scale, and the repulsive interactions generated by the charges along the chains. Here we present a hybrid Monte Carlo self-consistent field (MCSCF) approach to model such hydrogels. The SCF formalism is used to evaluate the conformational properties of the chains, implementing a freely-jointed chain model, in between the crosslinks. The Monte Carlo simulation method is used to sample the (restricted) translational degrees of freedom of the crosslinks in the gel. We consider the case that the polymers in the gel have an affinity for a surface positioned at the edge of the simulation volume. From the surface, the polymer density decays as a power-law, with an exponent close to $-4 / 3$, to the gel-density. The gel features relatively large density fluctuations, which is natural for a gel with a low density ( $\varphi \approx 0.035$ ), a low degree of crosslinking (average of three chain parts per crosslink) and relatively long chains $(N=50)$ in between the crosslinks. Some parts of the gel can break loose from the gel and sample the adjoining volume. Representative snapshots show large density fluctuations, which explains the large pore size distribution observed in experimental counterparts.

This chapter was originally published as: "Hybrid Monte Carlo Self-Consistent Field Approach to Model a Thin Layer of a Polyelectrolyte Gel near an Adsorbing Surface",F. A. M. Leermakers, J. Bergsma and J. van der Gucht , The Journal of Physical Chemistry A, 2012, 116, 6574-6581 


\section{Introduction}

This chapter was written in honour of the scientific career of Herman van Leeuwen for the occasion of his 65th birthday. Herman van Leeuwen dedicated a large part of his time to find ways to measure metal ions in solution and to model the fate of these systems in real life applications. $\frac{6162}{}$ Considering the adverse effects on the environment of some of these ions, insight in the speciation and availability in natural environments is now recognized as an essential ingredient to evolve to a sustainable future. 63

DET (Diffusion Equilibrium in Thin films) and DGT (Diffusive Gradients in Thin films) are analytical techniques to measure the metal ion concentration in, e.g., natural environments. ${ }^{64} 67$ Both techniques make use of a thin hydrogel layer, e.g. composed of crosslinked polyacrylamide. In DET one measures the equilibrium uptake of metals in such a gel. In DGT the gel layer is in contact with a sample solution and placed on top of a perfect sink (a strongly complexing resin). Classically, one expects that the metal ions partition into the gel and that complexing species, such as particles and humic acids can not. When the dimension of the gel (which must be larger than the diffusion distance of the metal species) and the diffusion constants of the metals in the gel are known, one can deduce the metal concentration in the sample solution from the time dependent accumulation of metals in the sink. Despite its wide application, the DGT method has several problems. More specifically, Herman van Leeuwen et al. showed that large particles, much larger than anticipated, can penetrate into the gel and, e.g., humic acids particles could be found in the gel in practical situations. $\frac{38139}{}$ The binding of metals to these internalized particles complicates the interpretation of DGT significantly. It is argued that a better understanding of hydrogels is necessary to optimize these techniques.

In this chapter we show a modelling approach which enables us to study physically crosslinked polyelectrolyte gels next to an adsorbing interface. From a modelling perspective physical gels, which allow redistribution of the chains over the crosslinks, are preferred over chemically crosslinked gels, wherefore redistribution is not possible. Heterogeneities in chemically cross-linked gels may be attributed to non-ideal conditions in the production phase and this invariably masks any in- 
trinsic inhomogeneities. Physical gels can equilibrate their structure and therefore inhomogeneities and other physical properties are more unambiguously interpreted. One of the aims of this work is to unravel relevant information about gels, which may be used to better understand the mentioned pitfalls of DGT. We further justify our work by pointing to the significant scientific challenges to model polymer gels and interfacially bound gels in particular.

Polymers tend to occupy the total volume of the system (dissolve) if the solvent quality is good. ${ }^{[68}$ Polymers that have chargeable monomers, polyelectrolytes, are an example of polymers that dissolve in water. When polyelectrolytes are crosslinked, as occurs in polyelectrolyte gels, the translational mobility is restricted and then these gels will occupy just a part of the available volume. Such a gel will still solvate itself with water, e.g., when the monomers dissociate. Most of the counterions, however, have to remain in the gel to ensure the local electroneutrality. The osmotic pressure of these ions decreases when the gel takes up more volume. Hence there is a driving force for the gel to increase its size. With an increasing amount of solvent in the gel, the distance between crosslinks increases. The chain parts between crosslinks eventually become stretched compared to their unperturbed radius of gyration. Such a stretched state is characterized by a relatively low value for the conformational entropy $\frac{68}{6}$ This counteracts the swelling. As a result there is an optimal amount of water in such a gel.

A gel with an optimal amount of solvent has a given charge density and as a result develops a so-called Donnan potential. ${ }^{69}$ The Donnan potential $\Delta \Psi$ is related to the concentration of ions in the gel $c_{i}$ compared to that in the outer solution $c_{i}^{b}$, where we use the super index $b$ to refer to the bulk (outer solution next to the gel):

$$
\frac{c_{i}}{c_{i}^{b}}=\exp \left(-\frac{v_{i} e \Delta \Psi}{k_{B} T}\right)
$$

where it is understood that the internal concentration of counterions $c_{i}$ equals the charge density of the gel and the co-ions (local electroneutrality condition). Eqn 3.1 is only reasonable when there are no further specific interactions of the ions that disturb this ideal Boltzmann equilibrium. A Donnan potential is well defined, obviously, when the gel is 
large compared to the electrostatic length scale set by the Debye length $\kappa^{-1} \propto\left(\sum_{i} c_{i}^{b} v_{i}^{2}\right)^{-1 / 2}$. For negatively charged polyelectrolyte gels, the Donnan potential $\Delta \Psi<0$ and thus the positive ions, such as metal ions with a charge $v_{i}>0$ accumulate in the gel. Here we focus on such negatively charged gels.

In DGT one uses a semi-macroscopic layer of water-soluble polymers next to a surface. $\frac{64}{67}$ This layer is thick, compared to the radius of gyration of the chains that are used, but still thin from a macroscopic perspective. A popular way to form significant polymer layers next to a surface is to make use of a polymer brush.70 In a polymer brush the chains are grafted by one of the chain ends to the surface, and the thickness of the brush becomes of order of the length of the chains. Such a brush layer however, rarely becomes thick enough for DGT applications. When the chains are crosslinked to each other, as in a gel, one does not need to covalently bind the chains to the surface to have a polymer layer that exceeds the radius of gyration of its chains as the thickness of this layer is an adjustable parameter. Some physical adsorption onto an underlaying surface is sufficient to effectively immobilize the gel. In this work we consider the case that the polymers that form the gel have an affinity for the surface so that the gel remains physically attached to it.

Because of the significant computational challenges there are relatively few molecularly realistic modelling attempts to describe polymer gels in the literature. We know several molecular simulation results relevant for polymer gels including polyelectrolyte gels. ${ }^{71 / 74}$ For a given model, such simulations give accurate information. Unfortunately it is not easy to consider large systems. Typically one considers a set of crosslinked chains forming an ideal diamond-like network (mimicking chemically crosslinked gels) in a computational box with periodic boundary conditions, implemented by a primitive model (no explicit solvent). The target of such simulations is to unravel some bulk properties of a gel. The relatively small size of the simulation box imposes significant limitations as, e.g., density fluctuations can only occur on length scales smaller than the box size. Moreover the chains must remain connected to the crosslinks by their pre-set functionality. One of the reasons to introduce a new method in this field is to reach larger length scales, and thus to probe gel properties on the mesoscale. On 
top of this, our method is designed to deal with physical crosslinks.

On the other side of the modelling spectrum there are analytical approaches to polymer gels, dating back to Flory $\underline{68}$, wherein the density of the gel and some of its elastic properties are predicted using polymer statistics. Without doubt these analytical approaches contributed most to our current understanding of polymer gels. Anticipating the discussion below, we may argue that the analytical approaches did not reveal the importance of fluctuations in these systems. Here our interest is in a relatively thin layer of a gel next to an adsorbing surface. To the best of our knowledge, the modelling of this type of systems has not been attempted before in the literature.

The Self-Consistent Field (SCF) method and in particular the approach of Scheutjens and Fleer (SF-SCF), has a strong tradition in describing adsorbing polymers and polyelectrolyte chains from dilute

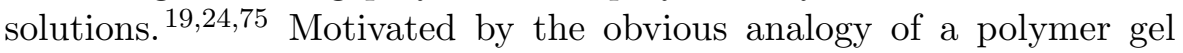
and a polymer brush, it is natural to take the SF-SCF theory as the starting point to model polyelectrolyte gels next to an adsorbing interface. The crosslinks, that are present inside the gel, present a special challenge to such an approach especially when these crosslinks have a physical rather than a chemical nature. Here we pick up this challenge by generalizing the SF-SCF formalism to account for the crosslinking inside gels using a Monte Carlo (MC) Ansatz. ${ }^{76}$ More specifically, we are going to generate relevant coordinates for the crosslinks with a MC protocol. These coordinates serve as boundary conditions for the chain parts whose conformational degrees of freedom are evaluated using the SF-SCF formalism. This MC-SCF hybrid is applied to a model system wherein a thin layer of a polyelectrolyte hydrogel is placed next to an adsorbing interface.

The remainder of this chapter is as follows. We start with a very brief description of the SCF model and the $\mathrm{MC}$ protocol to explain the relevant features of the MC-SCF hybrid. This is followed by specifying the model that has been implemented. In the results section we present the very first results from the MC-SCF method that prove its applicability for interfacially bound polyelectrolyte gels. The gels show large density fluctuations and as such support the results reported earlier by Herman van Leeuwen et al. in the context of DGT. $\stackrel{38 \mid 39}{ }$ 


\section{Modelling Considerations}

The two ingredients on which the MC-SCF hybrid is based are both well-known and well-documented. However the current hybrid method has not been reported before. Recently, this option was suggested in the context of polymer adsorption, as an improved way to account for inter- and intramolecular excluded-volume effects. ${ }^{52}$ On the one hand there is the MC method, which uses the Metropolis algorithm to generate relevant snapshots of the crosslink positions. On the other hand we follow the method of Scheutjens and Fleer ${ }^{19}$ which, on a mean-field level, considers inhomogeneous polymer systems. In the latter a specific discretization scheme is implemented, wherein space is subdivided into lattice sites and the chains are modelled as strings of segments that are placed in these lattice sites. Classically, the mean-field approximation is applied to layers of lattice sites. This results in systems wherein just one gradient direction remains (e.g. the case of polymer adsorption), or, in the case of a surface that is inhomogeneous in one direction, two gradient directions. The crosslinks in a gel, however have well defined coordinates. To determine the polymer distribution we therefore need a three-gradient SCF approach. The mean-field approximation, with three gradient directions, can now be considered as an average over time instead of over layers. The strategy to solve the SCF equations is well documented. ${ }^{24}$ This strategy can also be used for $3 \mathrm{D}$ gradients by using Hessian free (low memory) iteration schemes, such as conjugated gradients ${ }^{77}$, limited memory BFGS ${ }^{57}$, or Truncated Newton ${ }^{78}$ minimization strategies.

Both, MC and SCF operate on exactly the same volume. Here we consider a computation box with $V=L_{x} L_{y} L_{z}$ lattice sites. Introducing $x=1, \cdots, L_{x}, y=1, \cdots, L_{y}$ and $z=1, \cdots, L_{z}$ to specify the coordinates in this system, we take $L_{x}=L_{y}$ and assume periodic boundary conditions in both these directions. At $z=1$ an adsorbing boundary is placed, whereas at $z=L_{z}$ an inert boundary is present. Hence, we anticipate that the gel is next to the $z=1$ boundary and that the solution wherein the polymer density is low is close to the $z=L_{z}$ boundary. 


\section{Monte Carlo Aspects}

As in classical MC simulations, we introduce trial moves to change internal degrees of freedom of the system and apply acceptance rules such that the Boltzmann equilibrium is generated. ${ }^{[76}$ We discuss these aspects in order.

Let us first focus on the crosslink points. There are $j=1, \cdots, J$ such points, each specifying a cube with a volume of 8 sites (that is two lattice sites in each direction) with corners $\mathbf{r}_{j} 1=\left(x_{j}, y_{j}, z_{j}\right)$ and $\mathbf{r}_{j} 2=\left(x_{j}+1, y_{j}+1, z_{j}+1\right)$. This encompasses a total of $8 J$ lattice sites. From the MC perspective we can identify $J$ 'particles'. The positions of these $J$ MC-particles are generated using so-called trial moves. We have implemented translational moves of the crosslink points over a discrete number of lattice sites in either the $x, y$ or $z$ direction. Hence a trial move is split up into (i) the random selection of one or more MC-particles (crosslink points). (ii) The translation of this MC particle in the random direction over (iii) a restricted distance (typically just a few lattice sites). If the trial move positions the 'particle' partly or completely out of the 'box' in the $x$ or $y$ direction, the 'particle' is inserted at the opposite end of the box (periodic boundary conditions). As only translational moves are implemented, the MC trajectory may look like that of a diffusing particle. We haste to mention that this is only pseudo-dynamic as the proper friction, the topological restrictions (entanglements) and the correct hydrodynamics are not accounted for.

If a part of the crosslink overlaps with the solid boundaries at $z=1$ or $z=L_{z}$ the trial move is rejected. Also, when two crosslink sites overlap, the trial move is rejected. For the acceptance of a trial move in all other cases, one should know the change in the (free) energy, which is typically based on the pair interactions between all particle pairs. The unusual aspect of the current MC simulation is that the pair interaction is not a priori given.

At each step along the MC train we know the old coordinates, i.e., the positions of the MC particles before the trials are generated, and the corresponding old free energy $F_{\text {old }}$. The new coordinates, are passed on to the SCF module. On the SCF-level there are $I$ chains, each having $s=1, \cdots, N$ segments. These chain fragments can assume a large set of conformations, and the combined statistical weight of all 
possible and allowed conformations is collected in the so-called chain partition function. From this partition function it is possible to evaluate the free energy of the chains $F_{\text {new }}$. The link between the SCF and the MC part is that the first $s=1$ and last $s=N$ segment of each chain are constrained to be within the specified coordinates given by the $J$ crosslinks. As usual, the trial step is accepted if the free energy of the system decreases, that is when the free energy of the old configuration of crosslink points $F_{\text {old }}$ is higher than that of the new configuration $F_{\text {new }}$. The new configuration is accepted with a probability $0<P=\exp -\left(\Delta F / k_{B} T\right)<1$ when $\Delta F=F_{\text {new }}-F_{\text {old }}>0$ (importance sampling). Below we will mention in more detail the characteristic function and the governing free energy in the system.

The number of these trial steps as well as the distance over which the $\mathrm{MC}$ particles are moved is adjusted so that the acceptance probability is about $1 / e$.

\section{Self-Consistent Field Aspects}

From the above it is clear that the target of the SCF equations is to solve for the partition function of $I$ chains with the constraint that both chain ends occupy one of the coordinates specified by the $J$ sets of 8 coordinates. While doing so, the method gives detailed structural information on the system. Let us now assume that $\mathbf{r}_{k, j}$ crosslink sites are specified at the $k^{\text {th }}$ step in the MC-SCF procedure.

Within the SCF framework we can compute the volume fraction of segments $\varphi_{p}$ using the composition law, featuring two complementary end-point distribution functions. Because the polymers are symmetric we can write:

$$
\varphi_{p}(\mathbf{r})=\frac{I}{q_{p}} \frac{G(\mathbf{r} ; s) G(\mathbf{r} ; N-s+1)}{G(\mathbf{r})}
$$

wherein the end-point distributions are found by a propagator formalism 19

$$
G(\mathbf{r} ; s)=G(\mathbf{r}) \sum_{\mathbf{r}^{\prime}} \frac{1}{6} G\left(\mathbf{r}^{\prime} ; s-1\right)
$$

which implements a freely-jointed chain model for the chain conformations. The summation runs over all neighbouring sites of coordinate 
$\mathbf{r}=(x, y, z)$ assuming simple cubic ordering of the lattice sites: $\mathbf{r}^{\prime}=$ $(x-1, y, z),(x+1, y, z),(x, y-1, z),(x, y+1, z),(x, y, z-1),(x, y, z+1)$. In Eqn. 3.3 one can recognize a discrete version of a diffusion equation ${ }^{79}$, wherein $s$ is the equivalent of time and $\mathbf{r}$ the spatial coordinate. When $x-1$ or $x+1$ or $y-1$ or $y+1$ refer to coordinates outside the simulation volume, we implement periodic boundary conditions by adding or subtracting $L_{x}$, or adding or subtracting $L_{y}$, respectively, so that the new coordinate is within the box. In Eqn 3.2 the division by the free segment distribution function $G(\mathbf{r})=\exp -u_{p}(\mathbf{r})$ is needed because the weight of the segment at $\mathbf{r}$ is counted double, once in each endpoint distribution function. The normalization of Eqn 3.2 guarantees that there are exactly $I$ chains in the system. Here the single-chain partition function $q$ is evaluated as:

$$
q_{p}=\sum_{\mathbf{r}=\mathbf{r}_{k, j}} G(\mathbf{r} ; N)
$$

Finally, the propagators are initiated by

$$
G(\mathbf{r} ; 1)=G(\mathbf{r}) \delta_{\mathbf{r}, \mathbf{r}_{k, j}}
$$

with $\delta_{\mathbf{r}, \mathbf{r}_{k, j}}=1$ when $\mathbf{r}=\mathbf{r}_{k, j}$ and zero otherwise. These starting conditions guarantee that all chains have $s=1$ at one of the coordinates specified by the set of crosslinking coordinates. The use of the symmetry in the composition law (cf. Eqn 3.2) makes sure that also the other chain ends $(s=N)$ are at one of the crosslinking sites.

The above shows that the volume fractions of the polymer chains can be computed once the segment potentials for the polymer segments $u_{p}(\mathbf{r})$ are known. In addition we have segment potentials for the solvent $u_{s}(\mathbf{r})$ and segment potentials for the two ions $u_{\mathrm{Na}}(\mathbf{r})$ and $u_{\mathrm{Cl}}(\mathbf{r})$. In general we write for the (dimensionless) segment potential $u_{t}(\mathbf{r})$ with $t$ $=p, s, \mathrm{Na}$, or $\mathrm{Cl}$ :

$$
u_{t}(\mathbf{r})=\alpha(\mathbf{r})+\frac{v_{t} e \psi(\mathbf{r})}{k_{B} T}+\delta_{z, 2}^{t, p} \frac{\chi_{S}}{6}
$$

where $k_{B} T$ is the thermal energy. This equation contains three terms. The last one specifies the adsorption energy of the polymer segments 
$(t=p)$ sitting next to the adsorbing surface (at $z=1)\left(\delta_{z, 2}^{t, p}=1\right.$ when $t=p$ and $z=2$ and zero otherwise). The interaction parameter is specified by the Flory-Huggings $\chi_{S}$ parameter. The factor $1 / 6$ implements that in a simple cubic lattice only $1 / 6$ of the possible bonds is in the $z-1$ direction. The second term in Eqn 3.6 is discussed below. The term $\alpha(\mathbf{r})$ is used to implement the incompressibility of the sytem. This means that the sum of all volume fractions has to be unity:

$$
\varphi_{p}(\mathbf{r})+\varphi_{s}(\mathbf{r})+\varphi_{\mathrm{Na}}(\mathbf{r})+\varphi_{\mathrm{Cl}}(\mathbf{r})=1
$$

The value of $\alpha(\mathbf{r})$ is determined iteratively. When the sum of the volume fractions is larger than unity, $\alpha(\mathbf{r})$ is increased and if the sum is smaller $\alpha(\mathbf{r})$ is decreased. The volume fractions for the mobile monomeric components $t^{\prime}=s, \mathrm{Na}, \mathrm{Cl}$, are found by the Boltzmann equation:

$$
\varphi_{t^{\prime}}(\mathbf{r})=\varphi_{t^{\prime}}^{r} \exp -u_{t^{\prime}}(\mathbf{r})
$$

The summations over all segment weights give the respective partition functions $q_{t^{\prime}}=\sum_{\mathbf{r}} \exp -u_{t^{\prime}}(\mathbf{r})$. The number of molecules for the monomeric species are computed by $n_{t^{\prime}}=\sum_{\mathbf{r}} \varphi_{t^{\prime}}(\mathbf{r})$. It is understood that in the reference phase the volume fraction of the solvent and both ions are known $\left(\varphi_{t}^{r}\right)$. The reference phase, that does not contain any polymer, is in a (membrane) equilibrium with the gel and surrounding liquid.

The second term in Eqn 3.6 is the usual contribution due to the electrostatics where the reference for the electrostatic potential is also positioned in the mentioned reference phase. The charge $v_{t}$ is specified in the parameter section. The average charge at each specified coordinate is given by the mean-field Ansatz

$$
\rho(\mathbf{r})=\sum_{t} \varphi_{t}(\mathbf{r}) v_{t} e
$$

This charge density is used to evaluate the electrostatic potential by solving the Poisson equation

$$
\left(\frac{\partial^{2}}{\partial x^{2}}+\frac{\partial^{2}}{\partial y^{2}}+\frac{\partial^{2}}{\partial z^{2}}\right) \psi(\mathbf{r})=-\frac{\rho(\mathbf{r})}{\epsilon}
$$


wherein it is assumed that the dielectric permittivity $\epsilon$ is constant throughout the system and equal to that of the reference system. This differential equation is mapped onto the lattice and implemented using the periodic boundary conditions in $x$ and $y$ directions, similarly as has been outlined above for the chain statistics.

The above shows that it is possible to compute the segment potentials $u_{t}(\mathbf{r})$ when the segment volume fractions $\varphi_{t}(\mathbf{r})$ are known and similarly, that the volume fractions can be computed once the segment potentials are known. The fixed point of these equations is known as the self-consistent solution. For this situation the volume fractions both follow from and specify the potentials and vice versa the potentials both follow from and specify the volume fractions. A numerical procedure searches iteratively for this situation. When this SCF solution is available one can compute the free energy from

$$
F=-\log Q-\sum_{\mathbf{r}} \alpha(\mathbf{r})-\frac{1}{2} \sum_{\mathbf{r}} \rho(\mathbf{r}) \frac{\psi(\mathbf{r})}{k_{B} T}
$$

where $Q=\Pi_{t} q_{t}^{n_{t}} / n_{t}$ ! As the chemical potentials of the monomeric species can be evaluated from the composition of the reference phase, one can compute the so-called partial open free energy from

$$
F^{\mathrm{po}}=F-\sum_{t^{\prime}} n_{t^{\prime}} \mu_{t^{\prime}}
$$

Where the chemical potentials follow from the composition in the reference phase:

$$
\mu_{t^{\prime}} \equiv \frac{\mu_{t^{\prime}}}{k_{B} T}=\ln \varphi_{\mathrm{t}^{\prime}}^{r}
$$

The $F^{p o}$ is used to evaluate the MC acceptance rule, because we do not fix the number of small ions in the solution, but rather fix the chemical potential of the salt ions.

\section{Measurables of the MC-SCF Hybrid}

Before a new configuration of the crosslink points is generated we have saved the relevant system characteristics that are needed to compute the averages of measurable quantities in the system, so that $k=1, \cdots K$ 
snapshot results are available. At the end of the MC train, one can then flat average over the stored results, because each state of the system occurs with its proper frequency (Boltzmann weight) in these results. Here we focus on the volume fraction distribution $\varphi(z)$ averaged over the $K$ snapshots and averaged over the $x-y$ plane and the corresponding electrostatic potential profile $\psi(z)$. The free energy of the system $F^{\mathrm{po}}(k)$ gives information on the equilibration of the system in terms of the MC trajectory. On top of this we have snapshots of the system, from which we judge the polymer density fluctuations in the gel. Alternatively one can measure the distance between crosslinks and find a structure factor or radial distribution function. In the present system wherein the gel is surface bound and in which there exists a gel-solvent interface, the radial distribution function is not isotropic and the analysis of this function is left for a future publication.

\section{The Model and Model Parameters}

The target of this chapter is to present a typical result of an interfacially bound polyelectrolyte gel subject to physical crosslinks. The system volume $L_{x}=L_{y}=17$ and $L_{z}=100$, mimicking a thin slab of gel. The periodic boundaries in $x-y$ directions mimic the remainder of the gel. As mentioned already a crosslink point is defined by a sub-volume of two lattice sites in each direction (in total 8 sites). We have chosen for 12 crosslink points. In between the crosslinks there are monodisperse chains with $N-2=50$ segments (exclusive the two points that occupy crosslink sites; total length $N=52$ ). The size of a lattice site is set to the Bjerrum length, $b=0.6 \mathrm{~nm}$. On both sides of the system in the $z$ direction there exists an impenetrable and uncharged surfaces, namely at $z=1$ and $z=100$.

As each chain has two segments that have to sit on the specified crosslink sites, the ratio $I / J$ can not exceed the value of 4 , because $2 I$ can not exceed $8 J$. Values of $I / J<1$ (less than two chain-parts per crosslink) will not keep the chains immobilized in a gel, and then the chains are expected to sample the total volume of the system. We have chosen $I / J=3 / 2$ and thus we have $I=18$ chains in the system. Such relatively low value of the functionality typifies many experimental systems. (At each crosslink site, on average three chains come together). 
In many hydrogels there exists some charge along the chains. ${ }^{80}$ For this reason we have implemented the situation that a small fraction $1 / 20$ segments has a negative charge. For computational reasons we have distributed the charge over all segments. This means that the valency of a segment is set to $v_{p}=-0.05$.

Op top of this there exits a 1:1 electrolyte solution composed of some ideal salt which is completely inert with respect to the surface and the polymer chains (no specific adsorption effects). For ease of reference we refer to these ions as $\mathrm{Na}$ and $\mathrm{Cl}$. The concentration of these ions in the reference phase, that is in a system that is in equilibrium with the current system but which does not contain any polymer is set to $\varphi_{N a}^{r}=\varphi_{C l}^{r}=0.01(\approx 0.1 \mathrm{M})$.

The only non-zero interaction parameter in the system accounts for the affinity of the polymer segments for the surface at the lower bound in the $z$-direction. Here we have implemented $\chi_{S}=-6$, which effectively results in an adsorption energy of $1 k_{B} T$ per surface contact.

Typically we have implemented $10000 \mathrm{MC}$ steps, and by doing so have generated on the order of 4000 new configurations, wherein on average all crosslink points have been displaced somehow.

\section{Results and Discussion}

The MC-SCF hybrid brings together two computational disciplines. For this reason the discussion of what exactly the model intends to describe is necessary.

In the SCF part of the calculations, the full partition function of the 18 chains in the presence of the 1:1 electrolyte solution is evaluated. Hence, the volume fraction distributions are the result of the proper statistical weight of all possible and allowed freely-jointed chain conformations (time averaging). As all chains have two end segments that must be positioned onto one of the $8 \mathrm{~J}$ crosslink lattice sites, the chains cannot distribute randomly in the solution. On top of the crosslink constraints the chains have an affinity for the surface at the lower bound. However, only a fraction of the segments can find a place on this surface. It is expected that the polymers near the surface develop a layer composed of trains, loops and tails. The latter ones of course link to 
crosslink points in the neighbourhood of the surface. This mechanism is used to bind the gel to the surface.

In the MC part of the calculations, the focus is on the actual coordinates of 12 crosslink points in the system. Hence, at each instance just one set of coordinates is relevant. The averaging over many snapshots therefore is needed to find some ensemble average of the system of interest. On top of this, the MC-trajectory $k, \cdots, K$, gives detailed information on density fluctuations that exist in an adsorbed gel layer.

In the system there are $I$ chains (and thus $2 I$ segments that have to be on crosslink coordinates) and $J=12$ crosslink points (each containing 8 sites). As $I=18$ we have 36 segments on crosslinks. On average there are thus three chain parts that come together in a crosslink. We do not enforce that each crosslink has exactly 3 occupants. Fluctuations around this average are allowed. Of course it is also not forbidden that a chain forms a loop configuration and puts both of its ends on the same crosslink. Thus, there should be a finite probability that during the simulation a crosslink point will 'diffuse' out of the gel into the solution carrying one or more chains with it. This process is facilitated because we do not guarantee that on each crosslink site the number of chains that connect to it is an integer. Indeed, the ensemble average as searched for in the SCF calculations typically gives average numbers for these quantities, even for the crosslink points that 'float' in the solution.

There are various reasons for crosslink points to diffuse out of the gel. By doing so, the system can gain more translational degrees of freedom and this increases the entropy in the system. It also gives more space to the charged segments, resulting in a lower electrostatic repulsion between segments. However, chains that are partitioned onto detached crosslink points have to be in the 'loop' configuration. Inside the gel the chains can develop a multitude of bridge conformations. The latter is therefore favoured from a conformational entropy point of view.

One may expect that there exists a threshold ratio $I / J$ below which the chains sample the whole volume and the gel disintegrates, and above which the majority of chains is kept at all times in the gel. This threshold value may be referred to as a critical point. We do not know exactly what the critical value for $I / J$ is, although we may a posteriori conclude whether the system was apparently above or below this critical point.

In terms of volume, we consider a small system. Using the MC 


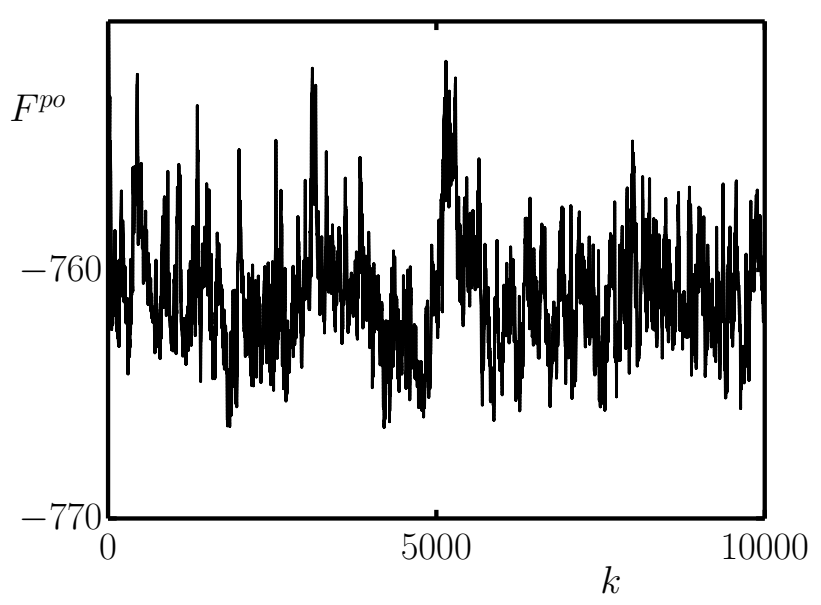

Figure 3.1: The free energy of the system in units of $k_{B} T$ as a function of the sequence $k$ (Monte Carlo Steps, MCS) generated by the MC trajectory.

protocol we generate a series of snapshots for the system. Each new set of crosslink coordinates gives a new distribution of chains in between the crosslinks. This is consistent with a physical gel, because in such a gel the crosslinks are not permanent. Again, we stress that it is not accurate to interpret this series of $\mathrm{MC}$ snapshots in a dynamical fashion. More accurately, one should have an ensemble in mind, which implies that each new configuration of the crosslinks, represents another part of the gel that sits on top of an infinitely large surface (ensemble). In this respect one may also interpret the model in terms of a chemically crosslinked gel. The fact that during the MC trajectory fragments of the gel detach from it means that due to some imperfect crosslinking of the gel, some (loose) fragments can diffuse out and sample the adjoining volume. Of course, a chemically crosslinked gel can be treated such that the loose parts are removed. In a physical gel it is impossible to remove all loose parts, because of the reversibility of the crosslinks. Hence, the current calculations resemble more naturally a physically crosslinked gel, but may also capture some aspects of chemically crosslinked gels.

In Figure 3.1 we present the free energy $F^{p o}$ of the system as function of the number of Monte Carlo steps $k$. After a short equilibration, 


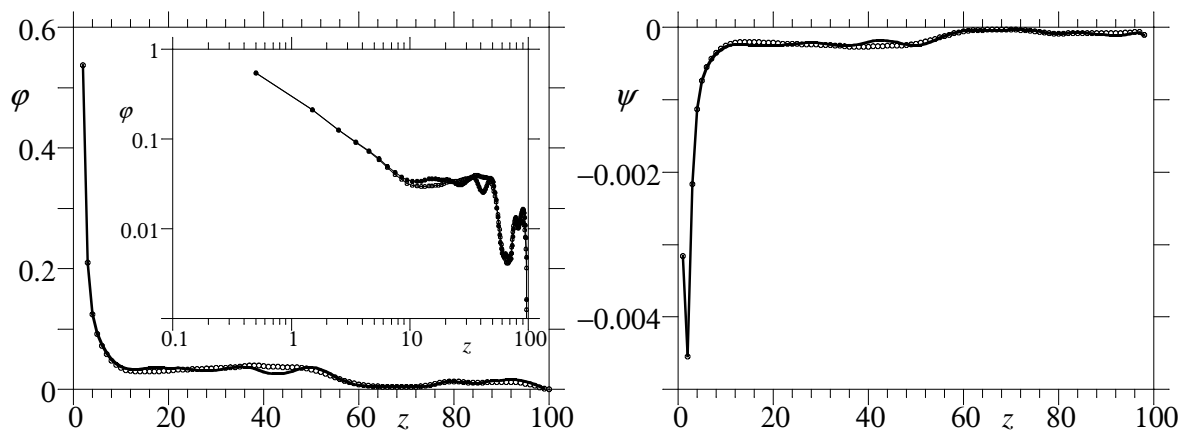

Figure 3.2: a) The volume fraction profile averaged over the $x-y$ plane as a function of the $z$-coordinate $\varphi_{p}(z)$. In the inset the same profile is given in double logarithmic coordinates (in the latter the $z$-direction was shift by 1.5 sites so that the $z$ coordinate represents the distance to the adsorbing wall in lattice units). b) The corresponding electrostatic potential $[\mathrm{V}]$ profile. In both graphs we present two data-sets. The data with the closed symbols are averaged over the last 5000 snapshots. The lines with the open symbols are averaged over all 10000 snapshots. From the difference between the curves one can estimate the accuracy.

the free energy fluctuates around a well-defined average value and the fluctuations are characterized by a standard deviation of 2.3 (in units of $\left.k_{B} T\right)$.

An averaging over the last 5000 (closed symbols) or over the full 10000 (open symbols) snapshots of the system, gives estimates for the volume fraction distribution of polymer segments in the $z$-direction, $\varphi_{p}(z)$. These volume fraction distributions are shown in fig. 3.2 a. The noise in the profiles is not extremely large proving the effectiveness of the averaging process.

As anticipated, there exists a high polymer density near the surface. This is due to adsorption of the polymer segments that take advantage of an adsorption energy of $-1 k_{B} T$ per surface contact. The width of the adsorption layer is exceptionally large. The volume fraction profile drops off to the gel-density in a power-law fashion (see inset). The power-law exponent of -1 fits the profile rather well. In the close proximity of the surface, the so-called proximal region, the decay is affected by the structure of the chains on the segment length scale. In this case 
there are crosslinks on the surface from which several chains originate. This gives a locally high polymer density and a non-trivial polymer density decay may have resulted from this. Somewhat further away from the surface $5<z<10$, that is in the central region of the adsorption layer, the profile falls off somewhat steeper hinting to the expected value of $-4 / 3$. Indeed this exponent is consistent with the radial polymer density decay in (polyelectrolyte) stars in good solvent 3158181 and it is also the value predicted by de Gennes for polymer adsorption lay-

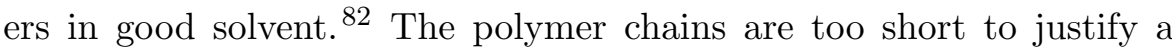
further analysis of the slope, but it is clear that the decay is well above the mean-field value of -2 for homopolymer adsorption from a good solvent. The fact that the apparent slope is significantly less negative than the mean-field value proves that in the current SCF method the intraand intermolecular excluded-volume effects are accounted for relatively accurately. $\frac{52}{52}$

Within the gel-layer the polymer density does not significantly depend on the $z$-coordinate. This is expected because the gel-phase coexists with the dilute phase and therefore should exist at a well-defined 'binodal' composition. The average density in the gel is estimated by averaging the volume fraction in the range $10<z<50$ which gives $\varphi_{g} \approx 0.035$. This is close to, but slightly below the overlap concentration $\varphi^{o v}=N^{-0.8} \approx 0.044$ for $N=50$. Apparently, the chains in the gel are stretched due to the osmotic pressure of the counterions in the gel and/or by the excluded-volume interactions of segments around the crosslink points. An estimated average distance $d$ between crosslinks follows from $\varphi_{g}=N / d^{3}$, which leads to $d \approx 11$. This is a large value compared to the Gaussian dimensions $\sqrt{N / 6} \approx 3$ and more compatible with $N^{0.6} \approx 10.5$ the scaling of a chain size in good solvent. The width of the gel-solvent and gel-surface interface is of order $d$, as expected. Above we argued that it is necessary that there exists a finite polymer concentration in the bulk phase. In the range $80<z<90$ a bulk polymer concentration of $\varphi_{p}^{b} \approx 0.012$ is found, whereas for $60<z<70$ the density dropped to $\approx 0.005$. The lower the density in the system, the harder it is to find a properly averaged value for it with MC. Averaging over the entire dilute phase gives $\varphi_{p}^{b} \approx 0.0085$ which is about 4 times lower than the polymer density in the gel. This difference is large enough to conclude that the gel coexists with the dilute phase. 
In Figure $3.2 \mathrm{~b}$ the average electrostatic potential $\psi(z)$ in the system is presented. As the chains are negatively charged the potential is negative throughout the system. Next to the surface, the potential is most negative because of the accumulation of charged segments at the boundary. In the bulk the potential also deviates from zero, because of the freely dispersed chain fragments that carry an electric double layer around them. (Note that the reference of the electrostatic potential is the reference phase, which contains only water and small ions at the specified volume fractions). In DET measurements, one uses a relatively thick layer of a hydrogel and in this case one only needs to focus on the (bulk) value of the Donnan potential inside the gel to account for an extra accumulation of metal ions in the gel. The surface potential effects can usually be ignored. ${ }^{83}$ In DGT measurements, one assumes that the gel is connected to a perfect sink. A large negative surface potential can be accounted for as part of the sink. Even though in this example the Donnan potential in the gel is very low, when it comes to evaluate the potential impact of it for practical situations we may not always ignore it. For example, when it comes to understand the incorporation of particles or humic acids in these gels the partition coefficient may be affected to a large extent because of the many charges per particle. $\frac{84}{}$

From the above it may be concluded that our system is not far from its critical value. In other words, the value of $I / J=3 / 2$ (the functionality) is just high enough to keep most of the chains in the gel. A slightly lower value is likely followed by the dispersion of the chains throughout the volume. From this point of view one should anticipate large density fluctuations in the system.

In Figure 3.3 we present 10 snapshots of the system, evenly sampled along the MC trajectory. In line with the observation that the system is not far from the critical point, we indeed observe large variations in crosslink configurations. Apparently, the crosslink points sample a relatively large part of the volume. From "time" to "time" a low polymer density also appears inside the gel phase. Such polymer voids translate in large pores. Indeed from the snapshots a rather dynamic picture emerges. This suggests that a rather disordered picture of a gel is often more realistic than an averaged one, as is shown by the $\varphi_{p}(z)$ profile, especially for polymer gels with a relatively low polymer density.

Returning to the context of DGT, one would like to characterize a gel 


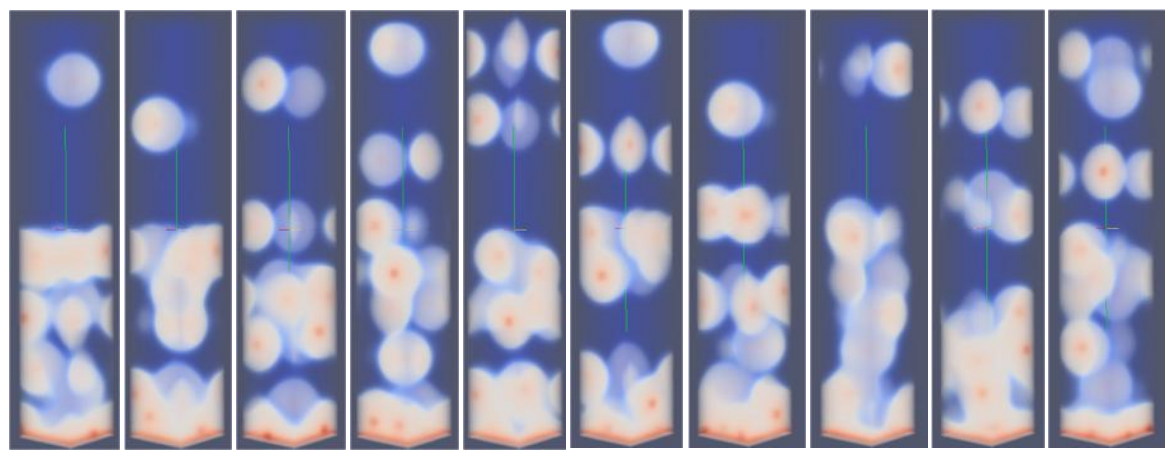

Figure 3.3: Ten snapshots of the system along the MC trajectory. The $z$-direction goes from bottom to top, and the $x-y$ directions point $45^{\circ}$ "left" and "right"-directions, respectively. The high polymer density is red, the lowest polymer density is blue.

by a pore size distribution and one would like this pore size distribution to be narrow. Motivated by the snapshots of fig. 3.3 which advocates a fluctuating point of view, it appears that the pore size distribution significantly exceeds the average distance between the crosslink points $d$. The limited size of our simulation volume however makes it impossible to determine a pore size distribution. On the one hand the maximum size of a pore is limited by the size of our system in the $x-y$ direction, while on the other hand the limited size of our system in the $x-y$ direction makes it easier for the gel to split as is shown in snapshot 6 of fig 3.3. Being close to the critical point, the fluctuations in density can become very large. A systematic analysis of the pore sizes as well as the influence of the finite size of the system on the pore size distribution, is directed to a future publication.

Complementary simulations/calculations, in which we varied the chain length, the functionality $I / J$ and the ionic strength, proved that the fluctuation picture is not an isolated result albeit that with an increase in $I / J$ the fluctuations diminished. With decreasing length of the chain parts the density in the gel increases, as expected from the gel theory. ${ }^{68}$ It is important to mention, however, that our conclusions are possibly affected by imperfections of the model and the use of a meanfield approximation in the SCF part of the calculations. Therefore firm 
conclusions on the structure of physical gels can only be drawn when these are backed up with experimental evidence. Of course, it is possible to use scattering techniques to get more informations on the type of density fluctuations in physically crosslinked gels. With reflectometry, it is possible to obtain similar data for interfacially bound gels. It would be interesting to correlate the current predictions with available scattering results in the literature.

\section{Conclusions}

We have introduced a Monte Carlo Self-Consistent Field (MC-SCF) theory to model a polyelectrolyte gel next to an adsorbing surface and discussed the preliminary results. The key idea of MC-SCF is that positional degrees of freedom of the crosslinks are generated using a MC procedure and the conformational degrees of freedom of the polymers in between crosslinks are accounted for by a freely-jointed chain model and optimized using the self-consistent field theory. As such, the pair interactions between the crosslinks are not a priori defined, but are computed using the SCF formalism. In this sense the MC-SCF hybrid can be seen as the polymer equivalent of $a b$ initio Car-Parrinello simulations in quantum mechanics. ${ }^{[55}$ We selected a system with a relatively low number of chains per crosslink. Such a gel is not far from the critical point and thus features large density fluctuations. We have predicted the structure of a small part of such a gel next to an adsorbing surface. The results point to a gel with a large pore size distribution exceeding by far the average distance between crosslinks. As such the calculations support recent findings that gels used in DGT measurements accommodate much larger particles than anticipated. 


\section{CHAPTER 4}

\section{A Hybrid Monte Carlo Self-Consistent Field Model of Physical gels of Telechelic Polymers.}

We developed a hybrid Monte Carlo self-consistent field technique to model physical gels composed of ABA triblock copolymers and gain insight into the structure of such gels. The associative A blocks of the polymers are confined to small volumes called nodes, while the B block can move freely as long as it is connected to the A blocks. A Monte Carlo algorithm is used to sample the node configurations on a lattice and Scheutjens-Fleer self-consistent field (SF-SCF) equations are used to determine the change in free energy. The advantage of this approach over more coarse-grained methods is that we do not need to predefine an interaction potential between the nodes. Using this MC-SCF hybrid simulation, we determined the radial distribution functions of the nodes, the structure factors and the osmotic compressibilities of the gels. For a high number of polymers per node and a solvent-B Flory-Huggins interaction parameter of 0.5 , phase separation is predicted. Because of the limited simulation volume, we could not establish a full phase diagram. For comparison, we performed coarse-grained MC simulations in which the nodes are modelled as single particles with pair potentials extracted from SF-SCF calculations. At intermediate concentrations, these simulations gave qualitatively similar results as the MC-SCF hybrid. However, at relatively low and high polymer volume fractions, the structure of the coarse-grained gels is significantly different because higher-order interactions between the nodes are not accounted for. Finally, we compare the predictions of the MC-SCF simulations with experimental and modelling data on telechelic polymer networks from literature.

This chapter has been published as: "A Hybrid Monte Carlo self-consistent field model of physical gels of telechelic polymers", J. Bergsma, F. A. M. Leermakers, J. M. Kleijn and J. van der Gucht, Journal of Chemical Theory and Computation, 14, 6532-6543, 2018 


\section{Introduction}

Here we describe a combination of the Scheutjens-Fleer self-consistent field theory with a Monte Carlo algorithm, which is used to simulate a gel network of symmetric telechelic polymers. These polymers have associative end-blocks, while the middle block is soluble. This combination leads to the formation of micelles in which the end-blocks associate in the core and the middle blocks form the corona. Such micelles are called flower-like micelles, with the core as the heart and the polymer loops as petals. At a sufficiently high concentration of micelles, the micellar cores are so close to each other that the ends of the polymers can be in different micelles, thus forming a bridge. Because the polymers can now form both loops and bridges, the number of possible conformations and thus the entropy increases. This increase in entropy gives an attractive contribution to the interaction between the micelles. If there are enough bridges to form a percolating network, a gel network is formed with the micellar cores as the nodes, as shown in Figure 1.3 .

Some researchers have reported that the attraction can become so strong that phase separation occurs ${ }^{8 / 45185}$; others however did not observe phase separation. $\frac{86}{}$ One reason why these experiments show different outcomes is that it is difficult to synthesize these polymers. Often the middle blocks show considerable polydispersity and not all polymer ends are functionalized. The latter will increase steric repulsion between the micelles and thus prevent phase separation. In computer simulations these problems can be avoided.

We assume that the binding energy of end-blocks to the micellar cores is so high that the concentration of free ends is negligible but still low enough that the ends can exchange between the cores. This allows the polymers to redistribute themselves over the micelles and form new bridges. This enables these gel networks to heal themselves when damaged. $47 / 48$

Because of these properties, telechelic polymers are applied in the paint industry to improve the rheological behaviour of paints. They can also be used as a gel material for gel electrophoresis. Furthermore, they are studied as a drug carrier for slow drug release. A hydrophobic drug can be dissolved in the core of the micelles. When a gel made of telechelic polymers is placed in the body, it will slowly release individ- 
ual micelles and thus the drug over time. An additional advantage in chemotherapy is that, due to the increased permeability of blood vessels in tumours, the micelles can accumulate in tumour tissue, which then receives a higher dose of the drug. 10

There have been many experimental studies on gels made of telechelic polymers. ${ }^{8 / 45|47 / 48| 85 \mid 86}$ The number of theoretical studies and simulations is, however, limited. $\frac{49187188}{}$ The length of the polymers makes it time-consuming to study these networks with molecular dynamics simulations even when coarse-grained bead and spring models are used for the chains. This is because a representative fraction of all the possible states of the system has to be sampled. As the polymers are large and can easily entangle, they diffuse slowly and it therefore takes a long time to reach and sample the equilibrium structure. One could choose to use even more coarse-grained models, for example, by simulating an entire micelle as a single particle. ${ }^{[9]}$ It is, however, difficult to describe the interaction potentials between the micelles properly, as the interactions are not necessarily pairwise additive. That is, the strength of the interaction between two micelles depends on the surroundings of the micelles, as shown in chapter 2 .

A solution to this problem is to employ a hybrid simulation technique which combines the benefits of particle simulations with the computational efficiency of self-consistent field calculations. Here, we combine the SF-SCF (Scheutjens-Fleer self-consistent field) method with a Monte Carlo algorithm. With the SF-SCF model, the free energy of a particular configuration of the nodes is calculated based on an average over all possible freely jointed chain conformations. This reduces the simulation time because the polymer configurations no longer need to be sampled individually. The positions of the nodes are sampled using a Monte Carlo algorithm that uses the free energy determined by the SF-SCF model to accept or reject the moves of the cores. In our model, we focus on the interactions between the nodes caused by polymers. The binding of the polymers to the nodes is done in a simplistic manner, since the interactions between the nodes are not influenced by the specific mechanism through which the polymer ends bind. Hence the results can be applied to a variety of gels of polymers with associative end groups, regardless of the exact binding mechanism.

The goals of this chapter are to demonstrate this hybrid Monte Carlo 
SF-SCF approach and to apply it to a system of polymer micelles in solution to gain insight in the structure of such systems. We compare the results with Monte Carlo simulations where the nodes, with polymers, have been coarse-grained to particles, with effective pair potentials as calculated in chapter 2, To further validate the method, the structure factors of the systems simulated by the Monte Carlo SF-SCF method are compared with experimental data found in literature.

It should be noted that this is not the first time that the SF-SCF theory is combined with a Monte Carlo algorithm. Previously, we showed some preliminary results for a charged polymer gel adsorbed on a wall, obtained with a model very similar to the one described in chapter 3 Furthermore, Charlaganov et al. $\frac{52}{1}$ used a combination of SF-SCF with Monte Carlo to study the depletion interaction of polymers near walls. They used approximate pair potentials to do a Monte Carlo simulation and subsequently used the self-consistent field theory to calculate a more accurate free energy and correct for the wrong weighing of the states. Potentially their method is more efficient, as the SF-SCF equations do not need to be solved for the rejected states. The rate at which the states of the system are visited is, however, determined by the free energy of the Monte Carlo simulation. If this free energy is not accurate, more steps are needed to reduce the noise level. This method is therefore only effective if a good approximation for the free energy can be determined. The more particles are present, the more accurate the interaction potential needs to be as the error would scale with the root of the number of particles. With our method, we do not need approximate potentials and the number of particles we could simulate is thus not limited in this way.

\section{Method}

First, we will explain the SF-SCF theory, specifically for the 3D simple cubic lattice that was used in the present study. It is similar to the method used in chapter 2, and some more details can be found there. Descriptions of the SF-SCF theory for other types of lattices can be found in chapter 2 and in the literature. ${ }^{20|54| 59 \mid 89}$ Next, we will show how we modelled the physical gel with the SF-SCF theory. Subsequently, 
the details of the Monte Carlo method will be described, and finally the methods for analysing the data will be discussed.

\section{SF-SCF Theory}

With the SF-SCF method, space is divided into lattice sites, which in the present study have a simple cubic ordering. Small molecules, such as solvent molecules, are represented by a single segment that has the size of one lattice site. Larger molecules, such as the polymers considered here, are represented by multiple segments. We assume that the segments of a polymer are connected like a freely jointed chain. Because we use a simple cubic lattice, the angle between subsequent segments can only be $180^{\circ}, 90^{\circ}$ or $0^{\circ}$. For $0^{\circ}$, the polymer is thus allowed to fold back onto itself. Segments adjacent to each other in the molecule of course still have to be next to each other on the lattice. The shortrange part of the interaction between different types of segments is quantified by the Flory-Huggins parameter $\chi$, which is half of the change in free energy when two segments are exchanged between homogeneous phases of each segment type.

It would be far too much work to generate all the ways in which the polymers can distribute themselves over the system one by one. So instead we determine the average distribution of the polymers over the system, i.e. we try to find the volume fractions for each segment type at each lattice site. These volume fractions can also be regarded as an average over time. This is done by generating all the possible polymer conformations, which are all the possible paths of the polymer chain on the lattice. Subsequently, the polymers are distributed over these conformations according to their Boltzmann weights. Because many of the conformations are nearly identical, this saves computation time. A disadvantage is that the interactions between the segments are calculated based on the average surroundings rather than on a specific configuration of the polymers, where with a configuration we mean a particular distribution of the polymers over the conformations.

The polymers will distribute themselves over the polymer conformations according to the Boltzmann weight $e^{-U_{c}}$ of these conformations. $U_{c}$ is the energy in units $k_{B} T$ of a particular polymer conformation $c$, given the average surroundings of this conformation. $U_{c}$ is the sum 
of the energy contributions of each segment. We call these contributions the segment potentials $u_{X}(\mathbf{r})$, where $X$ is the segment type and $\mathbf{r}$ is its location. These segment potentials $u$ are calculated from the volume fractions $\varphi$ of the various segment types in the neighbouring lattice sites, which in turn are calculated from the segment potentials. We repeat this iterative process until we find a self-consistent solution, in other words, until the segment potentials derived from the volume fractions are the same as those that were used to calculate these volume fractions. The segment potential of a segment of type $X$ is given by:

$$
u_{X}(\mathbf{r})=\sum_{Y} \chi_{X Y}\left\langle\varphi_{Y}(\mathbf{r})\right\rangle+\alpha(\mathbf{r})
$$

Here, the first term $\sum_{Y} \chi_{X Y}\left\langle\varphi_{Y}(\mathbf{r})\right\rangle$ describes the average interaction energy of a segment of type $X$, at position $\mathbf{r}$, with segments of types $Y$ in sites adjacent to position $\mathbf{r}, \chi_{X Y}$ is the Flory-Huggins parameter for the interaction between segments of type $X$ and $Y$, and $\left\langle\varphi_{Y}(\mathbf{r})\right\rangle$ is the average volume fraction of segment type $Y$ in all neighbouring lattice sites $\mathbf{r}^{\prime}$. The latter is given by:

$$
\left\langle\varphi_{Y}(\mathbf{r})\right\rangle=\frac{1}{Z} \sum_{\mathbf{r}^{\prime}} \varphi_{Y}\left(\mathbf{r}^{\prime}\right)
$$

where $Z$ is the number of neighbouring lattice sites. In our case, we have a simple cubic lattice and $Z=6$. We do not need to consider the interaction energy between segments of the same type as the FloryHuggins parameter $\chi_{X X}=0$ by definition.

The second term in Equation 4.1, $\alpha(\mathbf{r})$, is used to ensure that the sum of the volume fractions at each lattice site is unity. It has to increase when the sum of the volume fractions is larger than one and decrease when the sum is less than one. We chose to update $\alpha(\mathbf{r})$ with each iteration step as :

$$
\alpha_{\text {new }}(\mathbf{r})=\alpha_{\text {old }}(\mathbf{r})+\eta\left(\mathbf{1}-\frac{\mathbf{1}}{\sum_{\mathbf{X}} \varphi_{\mathbf{X}}(\mathbf{r})}\right)
$$

The factor $\eta=0.3$, which is small enough to prevent divergence. For the first iteration $\alpha_{\text {old }}(\mathbf{r})=\mathbf{0}$. 
The volume fraction of a segment $s$ of the polymer chain at lattice site $\mathbf{r}$ is given by the sum of the Boltzmann weights of all chain conformations $c$ that pass through $\mathbf{r}$ with segment $s$, multiplied with a normalization constant $C$ :

$$
\varphi_{s}(\mathbf{r})=C \sum_{c} e^{-U_{c(s, \mathbf{r})}}
$$

The normalization constant $C$ is the number of polymers $n$ divided by the partition function $q$, which is the sum of the Boltzmann weights of all polymer conformations of the polymer:

$$
C=\frac{n}{q}
$$

An efficient way to calculate $q$ and $\varphi_{s}$ is to use the propagator formalism.

The endpoint distribution function $G(\mathbf{r}, N+1)$ is the average Boltzmann weight of all chain conformations ending with segment $s=N+1$ on lattice site $\mathbf{r}$. In this study, we only allowed the polymers to start at coordinates that lie within the nodes. We therefore write the endpoint distribution function as $G\left(\mathbf{r}, N+1 \mid\left\{\mathbf{r}_{n}\right\}, 0\right)$ indicating that only the conformations starting with segment 0 within $\left\{\mathbf{r}_{n}\right\}$ contribute to the endpoint distribution function, as we have set the Boltzmann weight of all other polymer conformations to zero. Since the position of the last segment is the same for all conformations, we can move the contribution of the last segment $e^{-u_{X}(r)}$ outside this summation:

$$
G\left(\mathbf{r}, N+1 \mid\left\{\mathbf{r}_{n}\right\}, 0\right)=e^{-u_{X}(\mathbf{r})} \sum_{\mathbf{r}^{\prime}} \frac{1}{Z} G\left(\mathbf{r}^{\prime}, N \mid\left\{\mathbf{r}_{n}\right\}, 0\right)
$$

where $X$ is the segment type of segment $N+1$. The second part is the summation of the endpoint distribution functions of the chain without the last segment over all sites $r^{\prime}$ that are adjacent to $r$. Because only a fraction $\frac{1}{Z}$ of the conformations goes from site $r^{\prime}$ to $r$, we have to multiply the propagator in $r^{\prime}$ with $\frac{1}{Z}$. We can repeat this process until the first segment is reached. For this (starting) segment, the endpoint distribution function is simply $e^{-u_{X}(r)}$. In this way, we can calculate the entire endpoint distribution function.

With these endpoint distribution functions, we can calculate the 
volume fraction of each segment $s$ of the polymer according to:

$$
\begin{aligned}
\varphi_{s}(\mathbf{r}) & =C \frac{G\left(\mathbf{r}, s \mid\left\{\mathbf{r}_{n}\right\}, 0\right) G\left(\mathbf{r}, s \mid\left\{\mathbf{r}_{n}\right\}, N+1\right)}{e^{-u_{X}(r)}} \\
& =C \frac{G\left(\mathbf{r}, s \mid\left\{\mathbf{r}_{n}\right\}, 0\right) G\left(\mathbf{r}, N-s+1 \mid\left\{\mathbf{r}_{n}\right\}, 0\right)}{e^{-u_{X}(r)}}
\end{aligned}
$$

As the Boltzmann weight of segment $s$ is in both propagators, we need to divide by $e^{-u_{X}(r)}$. Because the polymers in our system are symmetric, we can save computation time by rewriting the first line of Equation 4.7 as the second line, so that only the propagators starting with segment 0 have to be calculated.

The overall volume fraction distribution of polymers is found by summing over all the polymer segments:

$$
\varphi(\mathbf{r})=\sum_{s=0}^{s=N+1} \varphi_{s}(\mathbf{r})
$$

The distribution of the monomeric solvent $S$ simply follows from the Boltzmann weight:

$$
\varphi_{S}(\mathbf{r})=C_{S} e^{-u_{S}(\mathbf{r})}
$$

When the segment potentials are normalized to zero in the pure solvent phase, which is in equilibrium with our system, $C_{S}=1$.

The Helmholtz energy, which is needed for the Monte Carlo moves, is given by:

$$
F=-\ln Q(u)-\sum_{\mathbf{r}} \alpha(\mathbf{r})
$$

where $Q$ is the partition function of the system. We calculate $Q$ using the ideal gas approximation:

$$
Q=\left(\frac{q_{P}^{n}}{n !}\right)\left(\frac{q_{S}^{n_{S}}}{n_{S} !}\right)
$$

The first term is the contribution from the polymers while the second term comes from the solvent. Here $n_{S}$ is the number of solvent molecules. The single molecule partition function of the polymers $q_{P}$ is calculated by summing the endpoint distribution function, over all 
positions $\mathbf{r}$.

$$
q_{P}=\sum_{\mathbf{r}} G\left(\mathbf{r}, N+1 \mid\left\{\mathbf{r}_{\mathbf{n}}\right\}, 0\right)
$$

The single molecule partition function of the solvent is given by $q_{S}=$ $\sum_{r} e^{-u_{S}(\mathbf{r})}$.

In the second term of Equation 4.10, we correct for the use of the Lagrange parameter. We have previously made two potentially conflicting assumptions. We have assumed that the system is incompressible and by defining $C_{S}=1$ we assume that the system is in equilibrium with a pure solvent phase. If, for example, we would place a solvophobic wall in our system, the volume fraction of the solvent would be lower near the wall than in the pure solvent. To make sure that the volume fraction next to the wall is the same as that in the pure solvent, which is required for an incompressible system, we introduced the extra potential $\alpha(\mathbf{r})$. There is of course no physical origin for this potential, and to get the correct Helmholtz energy for the given volume fractions, $\alpha(\mathbf{r})$ has to be subtracted from the Helmholtz energy.

\section{Gel Description within SF-SCF Theory}

Here and below we will express the Helmholtz energy in units $k_{B} T$ and measure the distances in lattice units. The polymers are represented by a chain of $N=50$ segments $B$, which represents the middle block, and one segment $A$ at each end, representing the end groups. We forced the end groups of these polymers to stay together, like in the micelles, by defining small volumes, called nodes, with a size of 3 by 3 by 3 lattice sites. By setting the Boltzmann weights for segments $A$ to zero outside the nodes, the end groups are forced to stay within the nodes. The set $\left\{\mathbf{r}_{n}\right\}$ thus encompasses all lattice sites that lie within the nodes. These nodes will be moved using a Monte Carlo scheme. Because the number of nodes that we can model is limited, we use periodic boundary conditions, so there is no interface in the system.

The following values for the various parameters were used as defaults in these experiments. The Flory-Huggins parameter $\chi$ was 0.4 and the polymer volume fraction $\varphi$ was 0.25 . The number of nodes $M$ was 125 with $f=5$ polymers per node, thus 625 polymers in total. We investigated the effect of changing several of these parameters on the 
structure of the gel. The volume fraction of the polymer $\varphi$ was varied from 0.5 to 0.031 . The effect of the Flory-Huggins parameter was studied by doing additional calculations for $\chi=0.0$ and $\chi=0.5$. Calculations were also done for 2.5 and 10 polymers per node. The number of polymer ends in each node is not fixed but can fluctuate around the average value depending on the statistical weights of the conformations starting and ending at this node. In practice, these fluctuations in the number of polymer ends in each node are limited due to the steric hindrance between the polymers. This is similar to real systems in which the number of polymers per node can also fluctuate. It also allows for slightly different compositions of the dilute and concentrated phases when phase separation occurs. In Figure 4.1 we show a few examples of the probability density function $f\left(N_{e}\right)$ of the number of end groups per node $N_{e}$. This distribution clearly becomes wider as the concentration increases. At high density, the steric hindrance between the polymers is less because the density around the nodes quickly drops to the bulk density and the polymers from the same node repel each other only over a short distance. It is therefore not so disadvantageous to put more than the average number of polymers on a node.

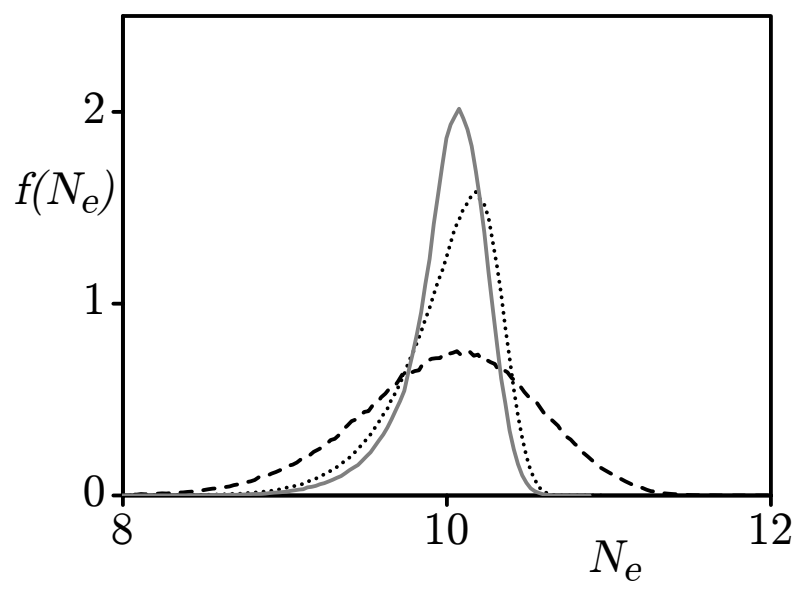

Figure 4.1: The probability density function $f\left(N_{e}\right)$ of the number of end groups per node $N_{e}$ for $N=125, \chi=0.4$ and $f=5$. $\phi=0.25$ (- $-), \phi=0.125(\cdots), \phi=0.063(-)$. 
To see to which extent the outcome of the simulation was affected by the limited number of nodes, we also did some simulations with 8, 27 and 64 nodes. For some systems the radial distribution function had not flattened out at a distance of half the box size. We therefore also did simulations with 512 nodes. A more detailed overview of the calculations performed as well as the code used for the GPU can be found online: https://pubs.acs.org/doi/suppl/10.1021/acs.jctc.7b01264

\section{Monte Carlo Protocol}

A basic Monte Carlo simulation consists of doing a Monte Carlo step, which is a trial move in the parameter space, and an acceptance rule which determines whether or not to accept the move based on the change in (free) energy. In our case, the trial moves consisted of picking a number of nodes at random and moving them by one lattice site in a random direction. A node could be selected multiple times during a single Monte Carlo step and can thus also move multiple sites. The number of nodes that are moved is adjusted during the equilibration part of the simulation, such that the acceptance ratio is about $25 \%$. After the nodes have been moved, the distribution of the polymers is calculated again and the new Helmholtz energy $F_{\text {new }}$ is compared to the old Helmholtz energy $F_{\text {old }}$. The reason for using the Helmholtz free energy is that when a node is moved, not only the interaction energy changes, but the conformational entropy of the polymers is changed as well. If the new Helmholtz energy $F_{\text {new }}$ is lower than the old Helmholtz energy $F_{\text {old }}$, the move is accepted. If it is higher, it is accepted with the probability:

$$
p_{\text {accept }}=e^{-\Delta F} \quad \Delta F=F_{\text {new }}-F_{\text {old }}
$$

At the start of the simulation, the nodes were ordered in a simple cubic ordering filling the the whole cubic simulation volume. We aimed to do $m=40.000$ Monte Carlo steps in each simulation. This is long enough for the system to equilibrate provided that the density remains homogeneous. To demonstrate that the system is equilibrated well within 40.000 steps, we show the SF-SCF Helmholtz energy as a function of the number of Monte Carlo steps in Figure 4.2 .

At first sight it may seem puzzling that the Helmholtz energy in- 


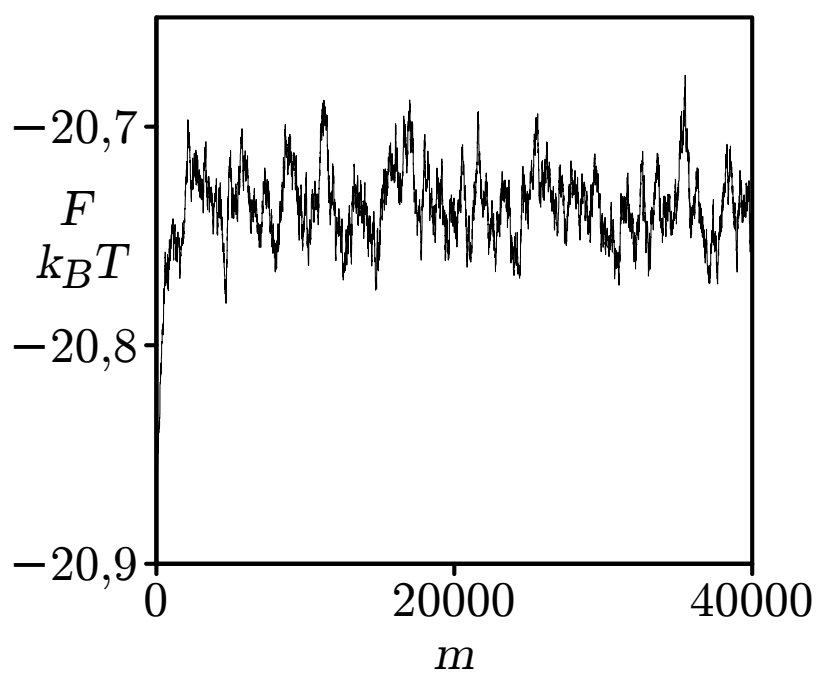

Figure 4.2: Helmholtz energy per polymer as a function of the number of Monte Carlo steps $m . \chi=0.4, \phi=0.25, f=5$ and $M=125$

creases as the system relaxes. The entropy of the nodes is, however, not included in the Helmholtz energy presented in Figure 4.2. At the start, the nodes are in a highly ordered state. By distributing themselves more randomly over the volume, the entropy of the nodes is increased. This results in a lower Helmholtz energy for the system as a whole even though the Helmholtz energy of the polymer chains has increased. In principle, the entropy of the nodes can be calculated from the radial distribution function and higher-order particle correlation functions using Green's entropy expansion. ${ }^{90}$ In our case, the three-particle correlation function was still rather noisy and it was therefore not possible to accurately determine the entropy of the nodes.

\section{Coarse-Grained Simulation}

To show that the hybrid Monte Carlo SF-SCF method describes the system better than Monte Carlo simulations with coarse-grained nodes, we performed Monte Carlo simulations with $M=125, f=5$ and $\chi=0.4$. In these simulations the nodes with their polymers have been 
coarse-grained to a single particle. We used an effective interaction potential, calculated as described in chapter 2 , as the interaction potential between these particles. To determine this effective pair potential, we first calculated the free energy per node for a simple cubic arrangement for different distances between the nodes. Subsequently, we calculated the effective pair potential so that it gives the correct free energy for all distances. The resulting potential is shown in Figure 4.3. The depth of the well is $0.33 k_{B} T$.

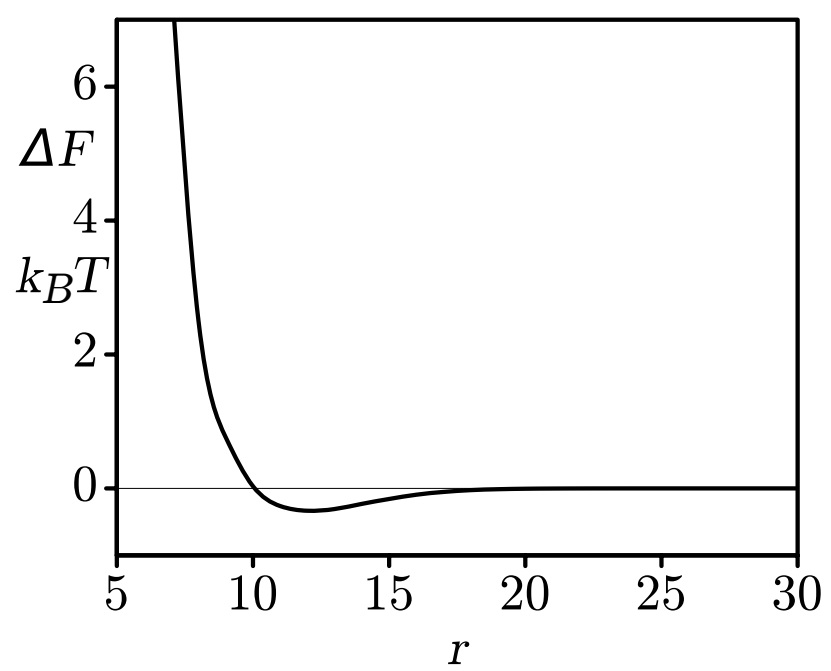

Figure 4.3: The effective pair potential used in the Monte Carlo simulations. The distance $r$ is measured in lattice sites.

\section{Data Analysis}

We calculated the radial distribution function of the nodes to see how much ordering there is in the system. This was done by splitting the range of possible interparticle distances in a number of subranges called bins. The width of these bins is $\mathrm{d} r$. Next, we loop over all particle pairs and Monte Carlo steps $m$ and count how many particle pairs have an interparticle distance that would fall in each bin $b$. "nint" indicates 
that number is rounded to the nearest integer.

$$
\begin{gathered}
b(r)=\sum_{m} \sum_{n_{1}} \sum_{n_{2}>n_{1}} \delta \quad \delta= \begin{cases}1 & r=\operatorname{nint}\left(\frac{\left|r_{n 1}-r_{n 2}\right|}{d r}\right) \\
0 & r \neq \operatorname{nint}\left(\frac{\left|r_{n 1}-r_{n 2}\right|}{d r}\right)\end{cases} \\
g(r)=\frac{2 V}{m M(M-1)} \frac{b(r)}{V_{r}}
\end{gathered}
$$

In this equation, $V$ is the volume in the number of lattice sites, $V_{r}$ is the number of lattice sites that fall within the bin $b(r)$ at radius $r$ and $m$ is the number of Monte Carlo steps over which the radial distribution function is averaged. $M$ is the number of nodes an $r_{n}$ is the position of the node.

To be able to compare the results of these simulations to experiments, we also calculated a structure factor $S(\xi)$ based on the radial distribution function using:

$$
S(\xi)=1+\frac{4 \pi \rho}{\xi} \int r(g(r)-1) \sin (\xi r) \mathrm{d} r
$$

In this equation, $g(r)$ is the radial distribution function, $r$ the distance, $\rho$ the number density of the nodes and $\xi$ the spatial frequency.

Due to the finite size of our system the radial distribution function does not go to exactly unity for large distances. This can, for example, be seen in Figure 4.4 where the dotted curve for $M=125$ stays just above unity. The explanation is that if a particle has an excluded volume, the volume remaining for the other $M-1$ particles is a bit smaller and the radial distribution function will be a little bit higher than unity far away from the particle. Similarly, if the interaction between the nodes is attractive, the concentration close to the node will be higher and far away it will be a bit lower. In that case the radial distribution far away will be a bit less than unity. As a result, a peak shows up around $\xi=0$ in the structure factor. As the osmotic compressibility is effectively determined by extrapolating the structure factor to zero, we need a way to suppress this peak at $\xi=0$.

Recently, Dawass et. a ${ }^{191}$ wrote an article comparing several methods for correcting some of these finite size effects. The best method according to them was the method of Ganguly and van der Veght. .92 


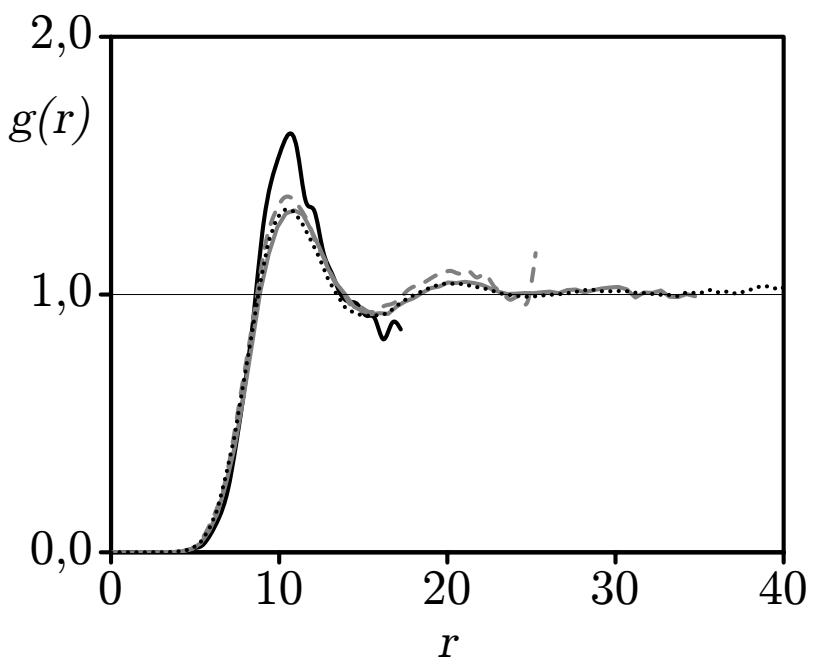

Figure 4.4: The effect of the number of nodes used in the simulation on the radial distribution function. The numbers of nodes in the system are: $8(-), 27(---), 64(-)$ and $125(\cdots) \cdot \chi=0.4, \phi=0.25$ and $f=5$.

They adjusted the radial distribution function at distance $r$ based on the excess amount within distance $r$. To us this did not seem optimal, as the excess amount fluctuates considerably as a function of the distance. As a first-order approximation, the value of the entire radial distribution function will be increased due to the local excluded volume of a particle. We therefore think that a correction that is more uniform would be better at approximating the real radial distribution function. The most logical thing to do would thus be to multiply the radial distribution with a small factor such that the radial distribution function goes to exactly 1 at long distances. For small simulation volumes, the radial distribution function is however not yet entirely flat at a distance of half the box size. It is thus not so easy to determine what the right correction factor is. Ideally, we get a smooth curve near a spatial frequency $\xi=0$. If we however get it wrong there, is a significant spike in the structure factor close to $\xi=0$. It therefore seemed reasonable to choose this correction factor such that the magnitude of 
the second derivative near $\xi=0$ is minimal, although a different derivative may work as well. To determine whether our method works, we simulated two systems, one with hard spheres and one with the effective interactions we use in the coarse-grained simulation. We compared the corrected radial distribution function and compressibility of boxes with 512 particles to those of a simulation box with 13824 particles to see if our method would give a useful correction of the radial distribution function. The corrected radial distribution functions for the systems with 512 particles give Kirkwood-Buff integrals that deviate less than $15 \%$ from the value of the large system, while the uncorrected values deviated as much as $60 \%$. For the hard sphere system with 512 particles, the value differs by about $5 \%$ from the theoretical value obtained with the K-equation of state ${ }^{93}$ and for the system with 13824 particles our correction reduced the deviation from $5 \%$ to $1.5 \%$. To our knowledge, this method has not been described in the literature and we hope to soon write a short communication in which we compare this method to other methods for correcting finite size effects. The values of the correction factors ranged from 0.987 to 1.008 . With this corrected radial distribution function, we calculated the osmotic compressibility $\kappa$ according to:

$$
\kappa=\frac{1+\rho \int 4 \pi r^{2}(g(r)-1) d r}{\rho k_{B} T}
$$

\section{Results and Discussion}

In Figure 4.5 an example of the simulation volume is shown. The nodes are clearly visible as red cubes with a slightly lighter core. Due to the steric repulsion between them, the polymers push each other away from the node and so drag their anchoring groups to the outside of the node. This results in a relatively low density within the core of the node.

In Figure 4.4, we show the radial distribution function for systems with different numbers of nodes $M$ and thus also different volumes. All other parameters have their default values. For $M=8$ and $M=27$, the radial distribution functions deviate significantly from the ones for $M=64$ and $M=125$, which are very similar. It thus seems that our default conditions using 125 nodes gives results that do not deviate too much from an infinite system, although there is still some effect of the 


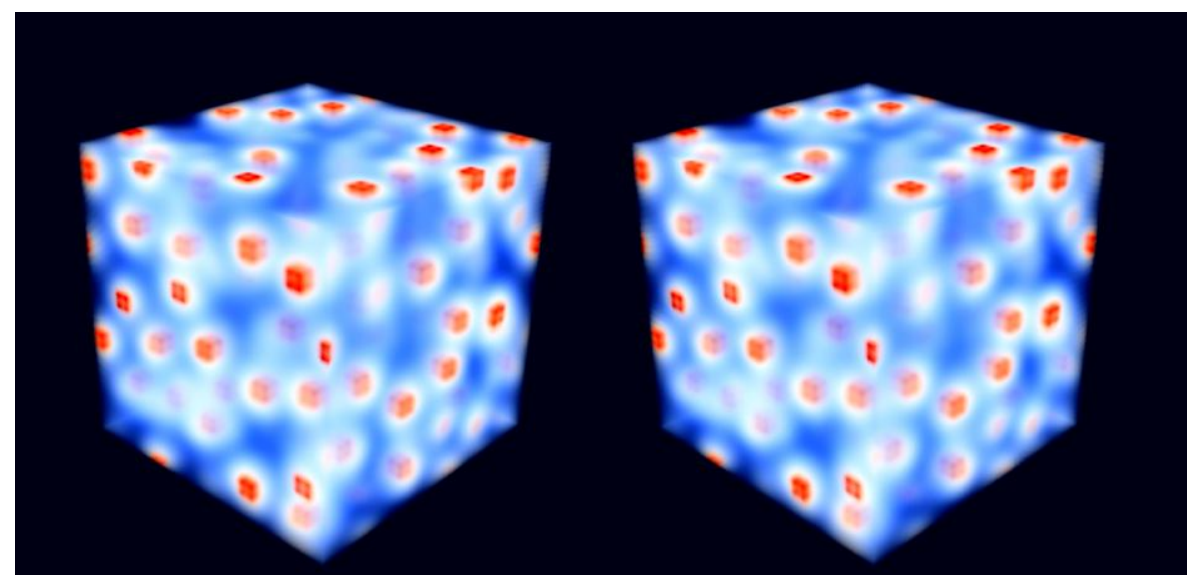

Figure 4.5: A 3D view of the default system. The nodes are coloured red. The polymer concentration decreases as the colour goes from red via white to blue.

limited size of the simulation volume. The system should not be much smaller, as the peak of the second coordination shell has barely ended at a distance equal to half the box size. With $\phi=0.5$ and $f=10$, the radial distribution function shows peaks well beyond half the box size and for this system as well as several others, we performed simulations with $M=512$ nodes. This still is not optimal, but the computational costs were too high to simulate even larger systems.

The dependence of the radial distribution function on the overall polymer volume fraction is shown in Figure 4.6. As the polymer volume fraction is increased from $\phi=0.125$ to $\phi=0.5$, the peak of the radial distribution function shifts inwards. Hence, at high concentrations the polymers are pressed into each other as there is not enough space to place all nodes at their optimal distances. As the volume fraction is reduced the distances between the nodes increase until the optimal distance is reached at a volume fraction of about $\phi=0.125$. There is no strong ordering in the sample, and only two relatively weak coordination shells are visible in the radial distribution function. The system is thus expected to behave like a liquid on time scales that are long compared to the relaxation time of an individual bridge.

At first sight, it may be surprising that the level of ordering of the 


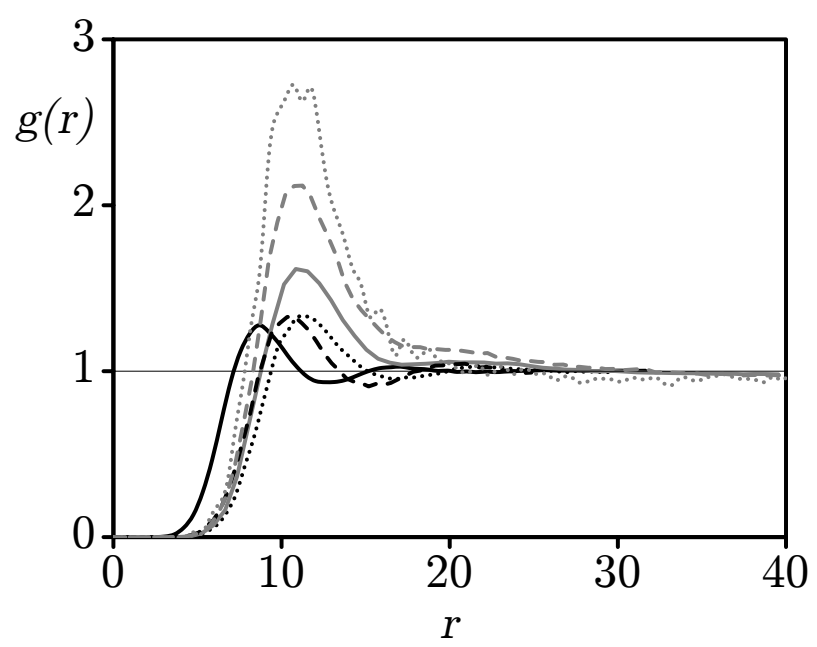

Figure 4.6: Effect of the polymer volume fraction on the radial distribution function. The tested volume fractions are: $\phi=0.50(-)$, $\phi=0.25(---), \phi=0.125(\cdots), \phi=0.063(-), \phi=0.031(---)$ and $\phi=0.0078(\cdots) . \chi=0.4, M=125, f=5$.

nodes does not increase with increasing node concentration. One would expect that due to the strong steric repulsion the nodes would order themselves. Similar to polymeric solutions, however, the environment starts to look more like a polymer melt, as the polymer concentration is increased. The polymers are therefore distributed more homogeneously over the volume. As a result, the steric hindrance experienced by the nodes will depend less on their position and the system can thus remain unordered.

As the polymer volume fraction is decreased from $\phi=0.125$, the peak of the first coordination shell rises, suggesting that the strength of the attraction increases. This is in line with chapter 2 where we found that the interaction between two nodes depends on their surroundings. As the system is diluted, the number of neighbouring nodes decreases and the attraction with the remaining neighbours increases. For dilute systems, the binding energy can be estimated by taking the logarithm of the peak height of the radial distribution function. In this case, the height is about 2.7 for $\phi=0.0078$, which corresponds to a binding 
energy of roughly $1 k_{B} T$. This binding energy and the position of the peak are the same as we found in chapter 2 .
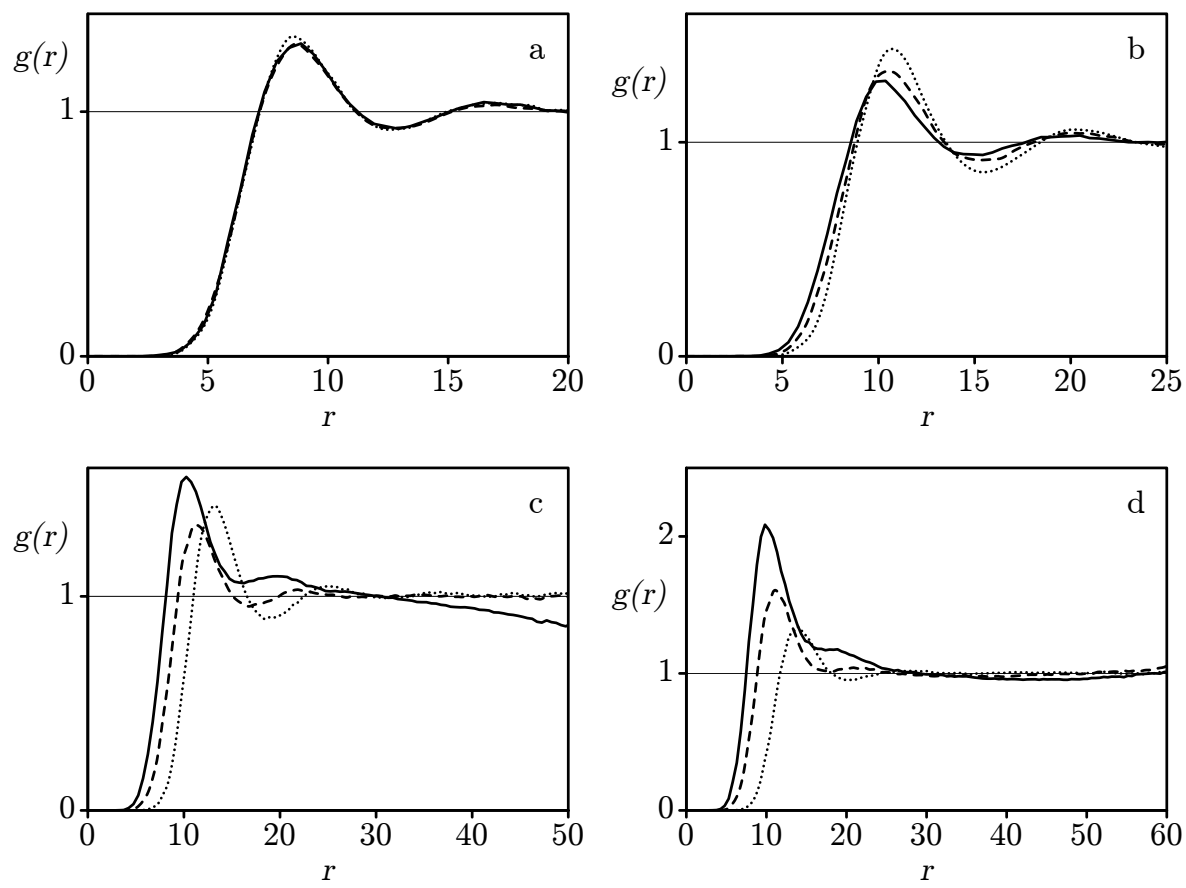

Figure 4.7: Effect of the solvent quality $\chi$ on the radial distribution function for different volume fractions: a) $\phi=0.50$, b) $\phi=0.25$, c) $\phi=0.125$ and d) $\phi=0.0625 . \quad \chi=0.5(-), \chi=0.4(---)$ and $\chi=0.0(\cdots) . M=125, f=5$. In sub-figures $\mathrm{c}$ and $\mathrm{d}$, part of the radial distribution lies beyond half the box size. The values in this range are displayed to show that phase separation occurs, although they are probably still be affected by the limited box size.

Let us next consider the effect of solvent quality. In Figure 4.7 radial distribution functions are shown for different values of $\chi$. At a volume fraction of $\phi=0.50$, shown in Figure $4.7 \mathrm{a}$, there is practically no difference between the different solvent qualities. At such a high polymer volume fraction, the swelling of the polymer corona does not significantly decrease the number of unfavourable interactions between 
the polymer segments because they would swell into the corona of the next micelle. The size of the micelles in a good solvent is therefore the same as that in a theta solvent and the radial distribution function is therefore also practically the same.

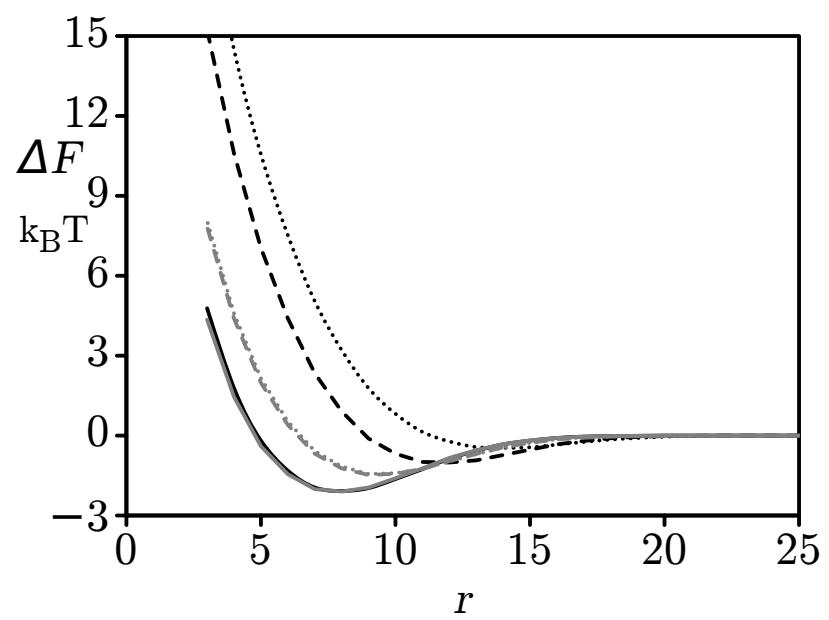

Figure 4.8: The effect of background polymers on the interaction potential between two nodes. $\chi=0.0$ black, $\chi=0.5$ grey. $\phi_{b}=0(\cdots)$, $\phi_{b}=0.1(---)$ and $\phi_{b}=0.5(-) . f=5$.

This is illustrated in Figure 4.8 where the interaction potential $\Delta F$ between two isolated nodes is plotted for different background polymer concentrations. At a background polymer volume fraction of $\phi_{b}=0.5$ the curves for $\chi=0.0$ and $\chi=0.5$ are practically the same.

As the polymer volume fraction is decreased to $\phi=0.25$, the radial distribution functions for the different solvent qualities start to differ. The peaks of the radial distribution functions shift outward, most strongly for the good solvent. For the theta solvent, the radial distribution function is otherwise similar to that at $\phi=0.50$. For a good solvent, the height of the peaks increases, as the steric repulsion is strongest in a good solvent and it thus gives the most ordered structure.

When the volume fraction is lowered further to $\phi=0.125$, we observe that for $\chi=0.5$ the radial distribution function no longer goes to unity at large distances. This is most likely because phase separation 
occurs: the cross section of the gel in Figure 4.9 clearly shows a dilute and a concentrated region.

In addition, the first peak for $\chi=0.5$ is higher than the peak for $\chi=0.0$. For $\chi=0.5$, the interactions are now attractive and they become stronger as the gel becomes more dilute, while for $\chi=0.0$ there is still a net repulsion between the nodes which decreases as the gel becomes more dilute.

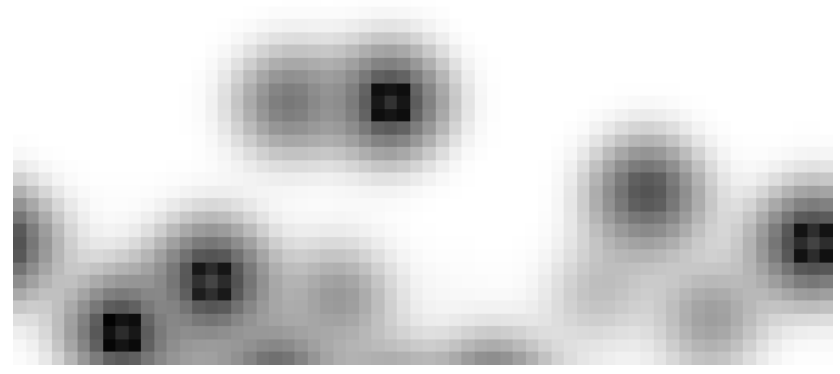

Figure 4.9: A cross section of the gel at $\chi=0.5, \phi=0.125, f=5$. The darker the colour the higher the polymer density. The maximum polymer volume fraction is about 0.75 . The nodes have clearly clumped together forming a dense region, which coexists with a dilute region with just a few micelles.

Finally, in Figure 4.7d, the polymer concentration has been lowered to $\phi=0.0625$. Now, the radial distribution function does go to 1 for $\chi=0.5$. This, however, does not mean that the system is already below the lower binodal. At the start of the simulation, the nodes are distributed homogeneously over the volume. They will initially clump 
together in small clusters. These clusters, however, diffuse much slower than individual nodes. It will thus take a long time before all the clusters and nodes have aggregated by diffusion and Ostwald ripening to form a dense phase. The simulation was therefore too short to fully equilibrate the system. The radial distribution function does show a slight dip at a distance of about 45 lattice sites, which is also visible for $\chi=0.4$. As the individual nodes and clusters diffuse around, they stick to other clusters. The concentration of micelles and other clusters near this cluster therefore decreases, leading to a zone with a relatively low concentration. This process may not only happen for complete phase separation but also in the case that the clusters have not yet reached their equilibrium size distribution. This is illustrated by the change in the radial distribution functions as the number of Monte Carlo steps is increased. The more Monte Carlo steps have been taken the further out the dip lies and the deeper it becomes. The system is thus not equilibrated within the simulated number of Monte Carlo steps. At the end of the simulation, there is also a clear void visible within the gel.

It is possible to improve the rate at which the system equilibrates by occasionally making Monte Carlo moves that displace micelles over large distances. We, however, intended to study the homogeneous phases of these micellar solutions and therefore did not implement such large Monte Carlo moves.

At a volume fraction of $\phi=0.0625$, the distance between the nodes is so large that, for all $\chi$, the interactions are no longer repulsive at the average intermicelle distance. The peak for $\chi=0.4$ is therefore higher than that at $\chi=0.0$ because the height of the peaks is now determined by the strength of the attraction between the micelles.

Now we turn to the effect of the number of polymers per node $f$, as shown in Figure 4.10. For $f=2.5$, the radial distribution function has just one peak just like a gas. In contrast, there are many peaks visible for $f=10$. For the highest concentration $\phi=0.5$, these peaks occur beyond half the box size. It is therefore likely that in this case the radial distribution function is still influenced by the size of the simulation volume. A striking difference between $f=10$ and the lower functionalities is that the height of the peaks increases as the concentration is increased from $\phi=0.25$ to $\phi=0.50$. This suggests that as the number of polymers increases, the micelles will behave more like hard particles 
Results and Discussion
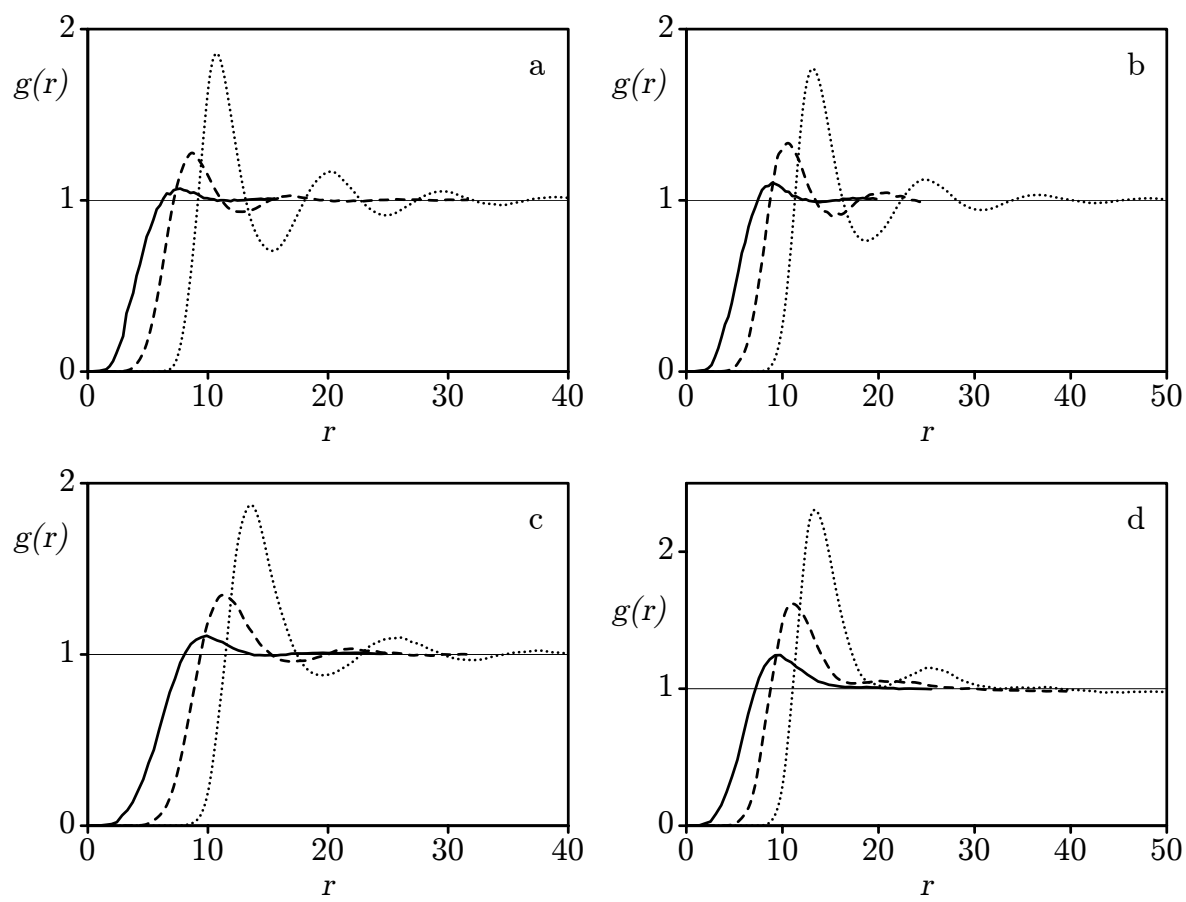

Figure 4.10: Effect of the number of polymers per node $f$ on the radial distribution function for different volume fractions: a) $\phi=0.50, \mathrm{~b})$ $\phi=0.25$, c) $\phi=0.125$ and d) $\phi=0.0625 . f=2.5(-), f=5(--$ -) and $f=10(\cdots) . \chi=0.4, M=125$, except for $f=5$ with $\phi=0.5$ and $f=10$ with $\phi=0.5$ or $\phi=0.25$ where $M=512$.

and crystallization may be possible for nodes with even more polymers. Figure $4.10 \mathrm{~d}$ shows that, for $f=10$, the radial distribution function drops a bit below unity at large distances, although the deviation is not as large as in Figure 4.7k. There are some interconnected cavities visible within the gel. It is therefore possible that this gel will also undergo phase separation even though this is not yet clearly visible. The number of Monte Carlo steps taken is relatively small and the gel may not have had enough "time" to phase separate.

Now that we have discussed the radial distribution functions for different parameters, we can compare them with the radial distribution 
functions calculated with Monte Carlo simulations in which we coarsegrained the nodes as single particles. In Figure 4.11, radial distribution functions from the MC-SCF simulations and the Monte Carlo simulations with effective pair potentials are shown.
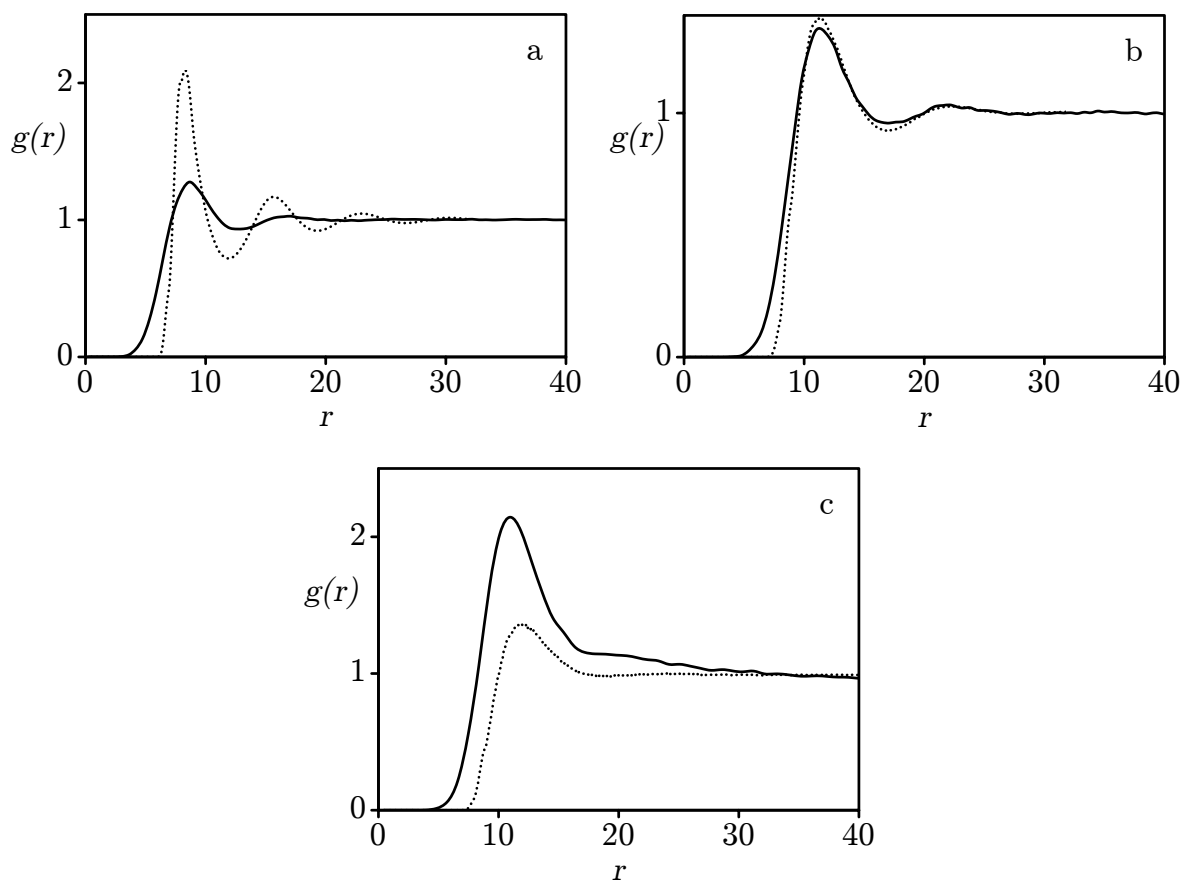

Figure 4.11: Comparison of the radial distribution functions for the MCSCF (-) and the normal MC simulation with effective pair potentials $(\cdots)$. The different volume fractions of the polymer are: a) $\phi=0.50$, b) $\phi=0.125$ and c) $\phi=0.031$. In all simulations $f=5$; in subfigure a, 512 particles were used and in subfigure b and c, 125 .

At high densities (see Figure 4.11a), the MC simulation with effective pair potential gives much sharper peaks than the MC-SCF model. This is probably caused by an overestimation of the repulsive force between the particles. When two nodes approach each other closely, the polymers can move out of the way if there are no other particles nearby. However, if the nodes have many close-by neighbours the polymers can 
not move out of the way and the repulsion is thus stronger. The MCSCF model can distinguish between these cases. A pairwise interaction, however, cannot, and instead an assumption has to be made about the surroundings of the nodes. In the way we determined the effective pair potential, it is assumed that the other nodes are at the same distance from the interacting nodes as the interacting nodes are from each other. When a particle is closer than the typical distance between a particle and its nearest neighbours, the average distance to the other nodes is underestimated and the repulsive force is too strong. Because of this, the nodes cannot approach each other as closely as in the MC-SCF model and therefore appear as harder particles, resulting in the sharper peaks.

At low concentrations, as seen in Figure 4.11k, the opposite problem arises. Here the peak of the first coordination shell is much higher for the MC-SCF model. Not only does the effective pair model overestimate the repulsion between the particles, it also underestimates the attraction. When a node already has many neighbours adding another one increases the number of polymer conformations relatively little compared to the total number of potential conformations. If instead a node has no neighbours, the relative increase in the number of polymer conformations is much larger. The change in free energy when a neighbour is added will therefore be larger when a node has fewer neighbours. The attraction at low concentration will therefore be stronger. With the effective pair potential it is assumed that there are neighbouring nodes at the same distance as the interacting nodes. This results in an underestimation of the attraction at low concentration.

At intermediate concentrations (Figure 4.11 b) the effective pair interaction gives roughly the same radial distribution function as the MC-SCF model, although the repulsion between the micelles is still overestimated at short ranges.

Based on these calculations, it is clear that a Monte Carlo simulation with a single pair potential does not correctly describe the behaviour of the nodes at a wide range of concentrations, although some improvement should be possible as the short-range repulsion appears too strong at all concentrations. An option would be to use a custom potential for each density. For systems in which the density remains homogeneous this would be an improvement. If the density is, however, not homo- 
geneous the result would be even worse than with the effective pair potential we have used here.

To validate our MC-SCF simulations, we need to make predictions that can be compared to experimentally obtained results. We therefore determined the structure factor and the osmotic compressibility $\kappa$ according to Equations 4.16 and 4.17 .


Figure 4.12: The structure factors calculated from the radial distribution functions for: a) $\chi=0 f=5$, b) $\chi=0.4, f=5$, c) $\chi=0.5$, $f=5$ and d) $\chi=0.4, f=10 . \phi=0.50(-), \phi=0.25(--)$,

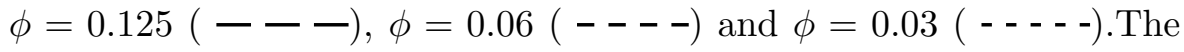
grey areas under the graphs indicate the $99 \%$ confidence interval.

The structure factors are shown in Figure 4.12 and the compressibility is plotted in Figure 4.13. Close to spatial frequency $\xi=0$, the uncertainty in the structure factor is relatively large. As the structure factor near $\xi=0$ is closely related to the compressibility, the accuracy 
with which the compressibility can be calculated is also limited.

In two of the presented cases, the structure factors are negative at $\xi=0$. For systems in equilibrium, this is physically unrealistic and it most likely results from the limited size of our simulation volume. In several other cases, increasing the number of nodes from 125 to 512 caused the structure factor to become positive. This would probably also be the case for these two systems if we could run the simulations with more Monte Carlo steps and nodes.
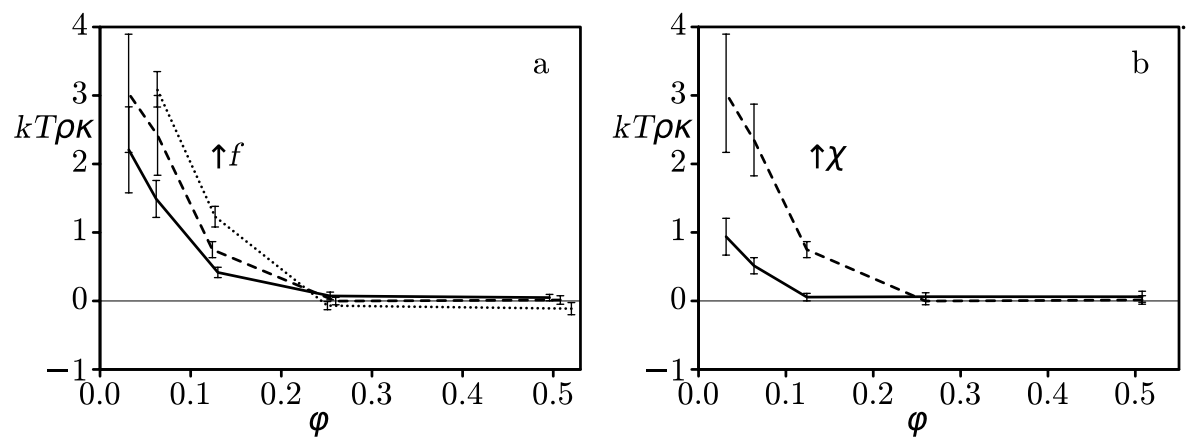

Figure 4.13: The osmotic compressibility relative to that of an ideal gas. a) For different numbers of polymers per node: $f=2.5$ (-), $f=5(---), f=10(\cdots)$. b) As a function of $\chi: \chi=0(-)$, $\chi=0.4(----)$. The error bars indicate the $99 \%$ confidence interval.)

The effect of the number of polymers per node $f$ on the osmotic compressibility (Equation 4.17) is shown in Figure 4.13a. The values shown are relative to the compressibility of an ideal gas with a particle concentration that is the same as the concentration of nodes in our simulations. At high polymer volume fractions, the steric repulsion of the polymer coronas prevents the nodes from coming close to each other and the osmotic compressibility is therefore much smaller than that of an ideal gas. At low concentrations, the attraction causes the nodes to form clusters and the osmotic compressibility is therefore higher than that of an ideal gas, because the number of freely moving particles is reduced. The relative compressibility gives a lower limit for the number of nodes that form a cluster. For $\chi=0.4, f=5$ and $\phi=0.03$, the average cluster size should be, for example, at least 3 . 
For $\phi=0.5$, the nodes with the fewest polymers per node seem to have the highest relative compressibility. The confidence intervals, however, still overlap so the difference is not significant. If we had instead looked at the real compressibility, the order would probably be reversed. The higher the number of polymers per node, the higher the concentration of polymers close to the nodes, while the polymer concentration halfway between the micelles is lower and thus the steric repulsion will be lower as well.

As the concentration is lowered, the order quickly changes. Because the nodes with more polymers have more attraction between them and thus form larger clusters, these systems are more compressible at low volume fractions.

In Figure $4.13 \mathrm{~b}$, the compressibility as a function of the FloryHuggins parameter $\chi$ is shown. As expected, the system with $\chi=0$ is the least compressible; as the corona swells the most, the steric repulsion is the strongest for this case. Above, we concluded, based on the radial distribution function, that phase separation occurs for the combination of $f=5$ and $\chi=0.5$. According to theory, the compressibility should therefore go to infinity. Our system, however, has a limited number of particles and therefore the value the compressibility can reach is limited. Furthermore, we used the entire radial distribution function to calculate the compressibilities. This, however, includes the part of the radial distribution function far away from the particle where it is below unity. This lowers the calculated value of the compressibility even further. The values obtained for $\chi=0.5$ and $\phi=0.13-0.03$ are thus incorrect and therefore not shown in Figure $4.13 \mathrm{~b}$.

One of the experimental studies in literature to which we can compare our results is by Filali et al. $\stackrel{45}{[4}$ They investigated a system of swollen surfactant micelles to which they added PEO polymers with hydrophobic end groups. Under the conditions used, the Flory-Huggins parameter for PEO is between $\chi=0.4$ and $\chi=0.5 \cdot{ }^{94 \mid 95}$ Although the polymers had about 120 Kuhn segments and were thus longer than the polymers we simulated, our results should show a fairly good match, as we found in chapter 2 that the effective pair potential is almost identical for 50 and 100 segments when the distance from the core is rescaled. In addition, the core of the micelles is larger than our nodes. However, compared to the volume of the coronas, the cores are still relatively small. 
The experimental results should therefore be in between our results for $\chi=0.4$ and $\chi=0.5$.

Filali et al. .45 observed phase separation for $f \geqslant 6$, which is not much higher than the $f=5$ for which we observed phase separation with $\chi=0.5$. In this respect, their results fit nicely with our findings.

The authors did not report the structure factor separately but did show the total scattering intensity. As the form factor goes to unity for small $\xi$, we should be able to make a qualitative comparison between our structure factor and their scattering intensities at small $\xi$. To get realistic values for our spatial frequency, we need to choose a value for the lattice size. We chose a value of $7.4 \mathbf{r A}$, because this coincides with the Kuhn length of PEG. .56

Based on the Daoud Cotton model[58, the system of Filali et al. with an oil droplet volume fraction of $7 \%$ should best match our simulations with a polymer volume fraction of $\phi=0.03$. When going from large $\xi$ to small $\xi$, there is a dip after the peak indicating the average distance between the nearest neighbours followed by a steep increase in both cases. These features are less pronounced than in our simulations. This is probaly because we used $f=5$, while the experimental system had on average four polymers per micelle. For higher concentrations, the structure factors do not match because the surfactant micelles in the experimental system are charged and repel each other at these concentrations.

François et al. published several experimental articles on telechelic polymers with PEO middle blocks. $\frac{196}{19}$ In contrast to us, they found cubic phases at high concentrations using X-ray scattering. 1 Since the number of polymers per micelle was not reported, a one-on-one comparison with our simulations is difficult. Probably the number of polymers per micelle is higher than in our simulations. This may explain why they observed a cubic phase and is corroborated by the fact that for longer middle blocks, for which the number of polymers per micelle is lower, crystallization was not found.

Another factor that may have contributed is that at least $10 \%$ of their polymers had only one functionalized end, which increases the repulsion between the micelles. They also observed phase separation for systems with relatively short middle blocks (PEO $M_{w} \leqslant 6000 \mathrm{~g} / \mathrm{mol}$ ) but not for polymers with long middle blocks $\left(M_{w} \geqslant 10000 \mathrm{~g} / \mathrm{mol}\right)$. As 
explained before, systems with longer polymers have fewer polymers per micelle and therefore less entropic attraction due to bridge formation.

Sprakel et al. studied the rheological and phase behaviour of solutions of the same type of telechelic polymers, both experimentally ${ }^{86}$ and with computer simulations. $\frac{44 \mid 49}{}$ In contrast to our simulation, they did not observe phase separation in their experimental study. ${ }^{[86}$ Although the number of polymers per micelle was not reported, it was probably larger than 6 , which was estimated by Filali et al. to be the lower boundary for phase separation. A possible explanation for not observing phase separation is that about $10 \%$ of the polymers had only one associative end group. This increases the steric repulsion between the micelles and the net attraction, which causes the phase separation, is thus reduced.

In a second paper Sprakel et al. $\stackrel{44}{ }$ addressed the phase behaviour of the system with a SF-SCF model in which the micelle is modelled in a 1D spherically symmetric system with a reflecting boundary condition. The number of polymers per micelle was not fixed, but instead the grand potential was optimized to determine $f$. Phase separation was predicted for all the combinations of middle block and end-block lengths they used in their study. The minimum number of polymers per micelle they found was about eight, but their polymers were much longer than those in our study. As they used $\chi=0.5$, all their systems lie above the line from $f=10$ with $\chi=0.4$ to $f=5$ with $\chi=0.5$. Their predictions are thus in line with what we found here.

In a third study ${ }^{49}$, they coarse-grained the micelles to single particles. In this case, no phase separation was found. They do not mention the Flory-Huggins parameter, but they wanted to reproduce the experimental system described above ${ }^{\sqrt{86}}$ and the value of $\chi$ should thus be between 0.4 and 0.5. Given that the simulated micelles have $f=25$ polymers each, phase separation would be expected based on our results. However, the interaction potentials in the coarse-grained model do not take into account that the attraction will increase with a decreasing number of neighbours. Moreover, a relatively small well depth of $0.38 k_{B} T$ was used, comparable to the well depth we found for $f=5$ and $\chi=0.4$ (about $0.34 k_{B} T$ ). Considering that in chapter 2 the well depth for an isolated pair of micelles roughly scales with $f^{0.5}$, the well depth expected for this system with $f=25$ would be about twice as 
large. These combined factors explain why they did not find phase separation from the coarse-grained modelling.

\section{Conclusion and Outlook}

We successfully combined a Monte Carlo algorithm with the ScheutjensFleer self-consistent field theory. With it, we were able to calculate the radial distribution function, structure factor and compressibilities for solutions and gels of ABA triblock copolymers with varying properties over a range of densities. For $f \leq 5$ polymers per node, we found, somewhat counterintuitively, that as the polymer volume fraction $\phi$ increases from $\phi=0.25$ to $\phi=0.5$ the amount of ordering in the system is decreased. We argue that this is because at high volume fractions the background concentration of the polymers of the other nodes becomes more homogeneous. The amount of steric repulsion therefore depends less on the position of the node. We further discovered that for $\chi=0.5$ and $f \geq 5$, phase separation occurs. We were, however, not able to determine the compositions of the coexisting phases as the number of simulated particles was small and there should thus be a considerable effect due to the interface. Simulating such a large volume that the effects of an interface would be negligible would take far too much computation time. To avoid the effect of the interface, the Gibbs ensemble $e^{97}$ can be used. Because we use a lattice, we can, however, not change the volume by arbitrary small steps but only by one lattice layer at a time. The larger the simulation volume, the larger the change in volume and thus in free energy will be. The chance that an exchange in volume would be accepted would therefore become smaller for larger and larger systems. This limits the system size we can use in combination with the Gibbs ensemble. Instead, it may be possible to "simulate" a Gibbs ensemble by simulating two volumes which would be representative for larger volumes of the simulated Gibbs ensemble. By moving particles in and out of the simulated volumes, the density could be adjusted to that of the simulated volumes of the Gibbs ensemble. To our knowledge, such an approach has not been described in literature yet. Another approach would be to coarse-grain the micelles while maintaining the dependence of the interacting potential on the surroundings of the in- 
teracting micelles. This method would also enable us to study dynamic properties of the system.

The limited system size may have affected some of our simulations at high polymer volume fractions where the radial distribution function had not completely flattened out by half the box size. The next generation of GPUs, however, promises to have 10 times more computation power as those we used. This allows larger simulation volumes and more Monte Carlo steps, making the Monte Carlo SCF hybrid model a feasible tool for future studies. By comparing the results of coarse-grained Monte Carlo simulations with those of the hybrid MCSCF model, we have shown the shortcomings of using only one pair potential to describe the interactions between the nodes. The MC-SCF hybrid method is therefore a useful tool to model systems of flower-like micelles and telechelic networks over a wide range of concentrations. 


\section{CHAPTER 5}

\section{Coarse-Grained Dendrimers in Good Solvent; Comparison of Monte Carlo Simulations, Self-Consistent Field Theory and a Hybrid Modelling Strategy}

The structural properties of dendrimers, with $g=1 \cdots 7$ generations, $f=2 \cdots 5$ spacers per branch point and spacer lengths $S=20$ and 50, are analysed with three methods that account differently for excluded volume correlations. (i) The cell model, where a dendrimer is anchored with the root segment in a spherically symmetric lattice and the dendrimer density is determined with the Scheutjens-Fleer self-consistent field (SF-SCF) approach. (ii) A hard sphere Monte Carlo (MC-FJC) model wherein all segments are connected by fixed length bonds and in between them (iii) the Monte Carlo self-consistent field (MC-SCF) hybrid, wherein the branch points are moved using MC and the polymer distribution is determined with SF-SCF. Comparing these gives information on the gain in accuracy when the intramolecular excluded-volume effects are gradually introduced. The focus is on the radius of gyration, the asphericity, the end and branch point distribution, and the overall radial density profiles. The cell model gave a slightly different scaling exponent for the radius of gyration (as expected for a mean field theory), but for large $f$ and $g$ values, it also wrongly predicts a bimodal distribution of the branch points closest to the centre. We ascribe these shortcomings of the cell model to the systematic overestimation of the long-range and underestimation of the short-range excluded volume effects within the SF-SCF theory. The qualitative differences between the MC-SCF and the MC-FJC model are much smaller. Localizing just a few of the segments thus largely compensates the shortcomings in the SF-SCF theory.

We submitted this chapter as: "Coarse-Grained Dendrimers in a Good Solvent; Comparison of Monte Carlo Simulations, Self-Consistent Field Theory and a Hybrid Modelling Strategy", J. Bergsma, F. A. M. Leermakers and J. van der Gucht, 2018 


\section{Introduction}

Inhomogeneous polymeric materials are of significant technological interest. These materials range from simple polymeric solutions to polymeric micelles and (double) gel networks. They can be used as absorbents, drug carriers in nanomedicine or to modify rheological behaviour, to name a just a few of their applications. To be able to design the polymers with which these materials can be created, it is important to understand how the properties of the polymers affect the behaviour of these materials. Mean field theory, is a popular way to study these systems as it is computationally very efficient. The accuracy of the theory is good for melts where excluded volume correlations are screened, but not so good in dilute cases where these correlations are important and we therefore do not know for sure which results of SCF theory can be trusted. More detailed methods such as Molecular dynamics and Monte Carlo simulations do take these correlations into account but sample only one configuration at a time and therefore take more computation time. There are relatively few strategies in between these limits.

In chapter 3 and 4 we have introduced a computational strategy to study spatially inhomogeneous polymeric systems wherein we combine the mean field Scheutjens Fleer self-consistent field theory with a Monte Carlo algorithm (MC-SCF). We used this method to simulate a physical gel of telechelic polymers. The polymer distributions were computed with the SF-SCF method, with the constraint that the polymer ends lie within so-called nodes (which are micellar-like cores that have specified locations). In turn, the nodes were moved with a Monte Carlo algorithm, which uses the SCF free energy of the system in the 'acceptance' step. Compared to classical SCF, calculations of this type are computationally expensive, as for each set of node positions the distribution of the telechelic polymers has to be recalculated. The computation time of the SCF-part of the simulation scales with the volume of the system, but not with the number of chains in it. This possibly counterintuitive feature makes the model relatively efficient for systems with a high polymer density such as the physical gels of telechelic polymers we studied previously.

The corresponding computations for a chemically cross-linked gel, 
however, require the strict coupling of chain ends to specified nodes (cross-links) and this makes the hybrid SCF method computationally even more challenging. In this case the computation time scales with the number of chains (or chain fragments) in the system. We show that, by using sub boxes and implementing the computations on a GPU, it is still feasible to develop and execute a hybrid MC-SCF method with explicit chemical cross-links. Here we implement this method not for a chemically cross-linked gel, but for dendrimer molecules, which may be seen as micro-gels.

One of the main reasons for developing hybrid MC-SCF methods is to investigate the shortcomings of the computationally extremely inexpensive SF-SCF method. With SF-SCF theory one uses a one-gradient spherical coordinate system (cell model) and the averaged properties of a system are predicted. In reality deviations from spherical symmetry exist, especially for molecules with few internal branches, most notably linear chains. The SF-SCF model cannot describe the shape of individual macromolecule conformations and properties that depend on these.

Other limitations of SCF exist. One issue is that every segment in SCF 'feels' an averaged local density instead of the real segment positions. A segment therefore does not feel that locally the density is higher due to segments that are close to it in the molecule, and whose positions are correlated. This allows conformations that fold back onto themselves. As a result the local excluded volume and thus the local stretching is underestimated.

At the same time the long range steric repulsion is overestimated within SCF, as it is assumed that all the segments are distributed homogeneously within each lattice layer. In reality the excluded volumes of segments that are close to each other in the molecule will overlap and the total excluded volume of the chain is thus smaller. The number of possible chain conformations is therefore higher as fewer chain conformations overlap.

In the newly formulated hybrid method, some of the segments are localized on a specific lattice site and moved with a Monte Carlo algorithm. The other segments are still treated with the SF-SCF machinery. This way the correlation in position between segments close to each other in the molecule is increased. This should cause segments 
close to each other in the molecule to feel their excluded volume better, and at the same time reduce the excluded volume effect between segments far away from each other in the molecule. This should alleviate the problems of the SF-SCF cell model, while still keeping the number of degrees of freedom that need to be sampled with the Monte Carlo algorithm limited.

In contrast to chapter 3 and 4 we now need to specify at which point a chain-fragment starts and ends, as the spacers in a dendrimer are attached to each other with chemical bonds. As a result, each spacer needs its own propagator. Because this propagator can only have non zero values in a small region near the branch points we introduce small sub boxes, so we only need to calculate the propagator in areas where it can have a non-negligible value. In the current implementation on a GPU, it is convenient to make all sub boxes the same size and shape. The size of the sub boxes is chosen in such a way that for all combinations of start and end-points the probability of the chain going out of the box is negligible. The efficiency can probably be improved by modifying the size of the box as a function of the positions of the start and end-points, and the propagator step.

We used dendrimers as a model system, to determine how effective this new model is with respect to accounting for excluded volume correlations. The reason for choosing dendrimers is that excluded volume interactions play an important role in them and there is already some literature to compare our findings with. $\frac{26 \mid 98-101}{10}$ We compared three different methods: (i) The cell model, that employs the classical SF-SCF equations (ii) the newly developed MC-SCF model, and (iii) as a reference, the hard sphere freely jointed chain model (MC-FJC) whose equilibrium conformations are generated by a Monte Carlo algorithm. We modelled systems with $f=2$ up to $f=5$ branches per node and up to $g=7$ generations. Results for a large number of generations and high degree of branching are rare in the literature. We include these because the MC-SCF method and especially the classical SF-SCF results are expected to improve for these molecules. Due to the many internal branches the molecules can not deviate much from the overall spherical geometry. The cell model, which assumes spherical symmetry, should thus be able to describe such a molecule well. 


\section{Method}

\section{Dendrimer Definition}

Dendrimers have a hierarchical structure and an illustration of such a molecule is presented in Figure 1.2. They 'start' with a central (root) segment. To this central segment $f$ spacers are connected each having $S$ segments. At the end of all these spacers there is a next segment that functions as a branch point. From this and each subsequent branch point there are $f-1$ new spacers of length $S$. The number of generations $g$ is defined as the number of branch points that are encountered starting from the centre and taking any path to the free ends of the molecule. In this process the central segment is also counted. At the end of the spacers of the final generation there is an extra terminal segment. This means that the length of the path from the centre to any of the free ends is $\mathcal{N}=g(S+1)+1$ segments long. The number of end-points is given by $n_{\mathrm{e}}=f(f-1)^{g-1}$. The number of spacers (arms) (including the outer ones) in the dendrimer equal $n_{\text {spacer }}=\sum_{j=1}^{g} f(f-1)^{j-1}$ and the number of branch points is $n_{\text {branch }}=1+\sum_{j=1}^{g-1} f(f-1)^{j-1}$. The total number of segments in the dendrimer is given by $N=n_{\text {branch }}+n_{\text {spacer }} S+n_{\mathrm{e}}$.

We have varied the functionality $f$ from 2 to 5 and used two values for the spacer length $S=20$ and $S=50$. For $f>2$ the number of generations we could model with the MC-SCF method was limited by the $3 \mathrm{~GB}$ of memory available on the GPU. It ranged from $g=7$ for $f=3$ and $n=20$ to $g=2$ for $f=5$ with $S=50$. This limitation could be lifted by implementing memory efficient algorithms. As this would have increased the computational cost even more we restricted the parameter space to the limiting values that could be treated by MC-SCF.

\section{Freely Jointed Chain Model with Excluded Volume (MC- FJC)}

As a reference model we use a dendrimer composed of hard sphere segments, each with diameter $d$, connect by bonds of length $l$ that can freely rotate. In the simulations the bond length is taken as the unit for distance, i.e., $l=1$ by definition. For ease of comparison with the SCF 
models we choose the diameter of the beads such that the maximum packing density of the beads is the same as in the SCF model. The diameter of the beads is therefore chosen as:

$$
d=\left(\frac{1}{4 \sqrt{2}}\right)^{\frac{1}{3}}=0.561
$$

We realise that when $l=1$, the beads overlap a bit and the real packing fraction is slightly higher. This is not a real issue because it is expected that a pre-factor is needed to match the outcomes of the SCF models with those of the MC-FJC model anyway.

We follow Chen et al. 100 who used a Monte Carlo protocol with pivot trial moves to generate new dendrimer configurations. In a pivot move a segment of the dendrimer is randomly selected. This segment and the outward part of the dendrimer attached to this segment are rotated around the segment one step closer to the centre along the contour of the dendrimer. The axis around which the rotation takes place is chosen randomly from the three perpendicular axis. The angle of rotation is randomly chosen from the range of 0 to $2 \pi$. If there is no overlap between the segments, after the rotation, the move is accepted. When the move is rejected the original conformation is counted again in the statistical averaging.

For dendrimers with a large number of segments the probability that pivot moves of segments close to the centre are accepted becomes very small. To improve the equilibration we therefore introduced an extra move for the segments between the centre of the dendrimer and the first branch points. This move consisted of rotating the segment by a random angle around the axis through the two segments to which it is attached. Whether this move or a pivot move is done, is determined randomly with each having a $50 \%$ chance. The probability that these segments were chosen was also doubled.

Due to the accumulation of rounding errors the bond lengths would over time start to deviate from unity. At regular intervals we therefore readjusted the bond lengths to unity. Occasionally this causes two segments to overlap. In that case the particle closest to the centre along the contour was moved away from the other particle until the distance between them was 1.00001. After this the bond lengths were 
again readjusted to length unity. The whole process was repeated till all bonds had length one and there was no overlap.

The procedure was tested with an ideal dendrimer (without excluded volume) and we found the correct average end-point distance and bond angle distribution indicating that the method was implemented correctly and that all configurations are visited with the correct a priori probability.

The simulations were started by generating a dendrimer with segments with zero radius. Starting from the previous segment we randomly generated a number of new positions at distance $l$. Subsequently, we chose the position that was most distant from the $s-1$ beads that were already in place. We defined an "inverse distance" $D\left(\mathbf{r}_{s}\right)$ as:

$$
D\left(\mathbf{r}_{s}\right)=\sum_{i=1}^{s-1} \frac{1}{\left\|\mathbf{r}_{i}-\mathbf{r}_{s}\right\|}
$$

and chose the position with the smallest $D\left(\mathbf{r}_{s}\right)$ value. Here $\mathbf{r}_{s}$ is the position of the bead $s$.

Once the dendrimer structure was generated, the Monte Carlo machinery was started. In addition to the standard Monte Carlo moves explained above, we increased the radius to 0.561 if this would not result in overlap with other beads. Once all the beads had the correct volume we did an equilibration run with a tenth of the number of steps used for data collection. The number of Monte Carlo steps taken varied between the different simulation runs.

\section{SF-SCF Theory}

The classical SF-SCF theory $19|25| 30|34| 102|103|$ has been described in the literature in detail. Here we will only focus on the main issues and ideas that are relevant for the modelling of dendrimers in the MC-SCF and cell model implementations.

Just as in the freely jointed chain model, macromolecules are described as a string of segments connected by bonds of fixed length, equal to the segment diameter. The chains 'live' in a discretised world, also known as a lattice. The size of the lattice sites is equal to the bond length, the segments thus fit exactly in the lattice sites. Segments that 
are connected in the molecule will be in neighbouring lattice sites. The longer ranged correlations between segment positions are ignored, this is also known as the Markov chain approximation. In contrast to the MC-FJC model, chain backfolding is thus allowed.

In this lattice a mean field approximation is applied. This means that the average volume fractions in a lattice site $\varphi(\mathbf{r})$ are determined based on the interactions with the average environment. Next to the volume fractions $\varphi(\mathbf{r})$ each lattice site also has a segment potential $u(\mathbf{r})$ for each segment type. The segment potential at coordinate $\mathbf{r}$ represents the energy needed to bring a segment from the bulk to coordinate $\mathbf{r}$, when this segment is not (yet) connected to other segments in a chain. For convenience the potentials are expressed in units $k_{B} T$.

The segment potentials implement the interactions between the segments. The segment-interactions are accounted for with the BraggWilliams mean field approximation and described by the Flory-Huggins $\chi$ interaction parameter. As we focus on good solvent conditions, $\chi=0$. In this case the only remaining contribution to the segment potential comes from the incompressibility condition. This means that the segment potentials are chosen such that the sum of the volume fractions of the solvent, $\varphi_{O}(\mathbf{r})$ and the dendrimer $\varphi_{D}(\mathbf{r})$ add up to unity, $\varphi_{D}(\mathbf{r})+\varphi_{O}(\mathbf{r})=1$ at each coordinate $\mathbf{r}$. To determine the values for the segment potentials we need to know the volume fractions. As the volume fractions in turn depend on the segment potentials we have a chicken and egg problem. To circumvent this we start with an initial guess for the segment potentials, usually $u(\mathbf{r})=0$. With this guess we can start to compute the volume fractions. This begins with the evaluation of so-called segment statistical weights $G(\mathbf{r})$, which are given by:

$$
G(\mathbf{r})=e^{-u(\mathrm{r})}
$$

The segment statistical weight is the contribution a segment at the specified coordinate would make to the statistical weight of polymer conformations that pass through this lattice site. The solvent consists of freely dispersed monomers and therefore their volume fractions are proportional to the segment statistical weights and are determined with:

$$
\varphi_{O}(\mathbf{r})=G(\mathbf{r})
$$


This is equivalent to $\varphi_{O}(\mathbf{r})=\frac{N_{O}}{q_{O}} G(\mathbf{r})$. The latter equation is numerically more stable. Here $N_{O}$ is the number of solvent molecules in the system and $q_{O}=\sum_{\mathbf{r}} G(\mathbf{r})$.

Determining the polymer segment distribution is more complicated. For this we have to combine statistical weights of sets of conformations of parts of the polymer. We use so-called end-point distribution functions $G\left(\mathbf{r}_{\mathbf{t}}, t \mid \mathbf{r}_{\mathbf{b}}, b\right)$ to do this. These contain the combined statistical weight of all possible and allowed conformations of a chain fragment beginning with segment number $b$ at coordinate $\mathbf{r}_{\mathbf{b}}$ and terminating with segment $t$ at coordinate $\mathbf{r}_{\mathbf{t}}$. These end-point distributions are generated using a propagator formalism which employs segment statistical weights as a key ingredient. The segment density, $\varphi(\mathbf{r}, s)$, for segment $s$ at coordinate $\mathbf{r}$ is found by combining all end-point distributions that can reach segment $s$. The details of this formalism depend on the molecular topology of the chains and on the constraints that are imposed. This information differs between the MC-SCF hybrid and the cell model. Therefore we give the details below in separate subsections.

Once we have determined the volume fractions, we can in turn calculate the segment potentials. If the sum of the volume fractions is larger than unity, we need to suppress the densities. This is implemented by increasing the potentials. The inverse should happen when the volume fractions are below unity. Hence, we can update the segment potential at each iteration $k+1$ from values found at iteration $k$ :

$$
u^{k+1}(\mathbf{r})=u^{k}(\mathbf{r})+\eta\left(1-\frac{1}{\varphi_{0}^{k}(\mathbf{r})+\varphi_{D}^{k}(\mathbf{r})}\right)
$$

where $\eta$ lies in the range $0<\eta<1$ and is chosen such that convergence is achieved. We can iterate over these equations until the sum of the volume fractions in each lattice site has converged to one.

In practice we use a target function that combines the incompressibility relation with a self-consistency of the potentials ${ }^{24}$ and more sophisticated algorithms which converge faster than a simple steepest descent algorithm. An example is the DIIS algorithm ${ }^{104}$ which converges routinely and efficiently in 10 to 100 iteration steps to the SCF solution to a precision of 7 significant digits. 


\section{MC-SCF}

In the MC-SCF model we use a 3D simple cubic lattice where the coordinates are given by $\mathbf{r}$. The total number of lattice sites in each simulation is given by $m^{3}$, where $m$ is the total number of sites in each of the three directions $x, y, z$. The idea is that we split up the dendrimer in spacers, which lie in between the branch points and between branch and end-points. For each dendrimer there are $n_{\text {spacer }}$ spacers for which at each point during the simulation the start and end segments have to lie next to the appropriate branch and end-points. To generate the initial positions of these branch and end-points we use a random walk on the lattice, which could be biased to move away from its starting position. We started from the root segment and did $S+1$ steps in a random direction to reach the position of the first generation of branch points. This process is repeated until finally the positions of the end-points have been determined. Obviously it is checked that the branch and end-points are not placed on top of each other. In a few cases this did not work and instead we remapped the positions of the branch and end-points from the MC-FJC model onto the lattice.

For each spacer $i$ we need to perform a separate propagator to generate the densities of this fragment $\varphi_{i}(\mathbf{r})$ from the segment potentials $u(\mathbf{r})$. There exists an excellent opportunity to do this in parallel because the calculation of the propagator of one spacer is independent of those of other spacers. It is also clear that we do not need to develop the propagator in the entire volume, as spacers can only contribute to the density in the vicinity of the branch and end-points they are connected to. Therefore we specify for each spacer $i$ a sub-volume $v_{i}=M^{3}$, called sub box, with sub-coordinates $R_{i}=\left(X_{i}, Y_{i}, Z_{i}\right)$ each running from $X_{i}, Y_{i}, Z_{i}=1, \cdots, M$. The position of this sub box is chosen such that the center of this sub-volume lies exactly between the branch/end-points.

It is clear that the sub box $M$ can be much smaller than the main box $m$. For $S=20$ the sub box size is $M=20$, for $S=50$ we used a sub box size of $M=37$. The latter is smaller than the maximum extension of the polymer. The required entropy-loss to stretch the polymer to this size is however very high and we did not observe stretching beyond 25 lattice sites. The small boxes have reflecting boundary conditions even though 
these conditions will not significantly influence the results because the sub box size $M$ is so large that conformations rarely 'hit' the sub box boundaries. For each sub box $i$, the sub box coordinates are filled with the segment potentials from the main volume: $u_{i}\left(\mathbf{R}_{\mathbf{i}}\right)=u\left(\mathbf{r}_{i}+\mathbf{R}_{i}\right) \forall \mathbf{R}_{i}$. Here $\mathbf{r}_{i}$ is the position of the origin of the sub box $i$ on the main lattice.

Also the position $\mathbf{r}_{\mathbf{0}}^{\mathbf{i}}$ of the first segment $s=0$ and the position $\mathbf{r}_{\mathbf{S}+\mathbf{1}}^{\mathbf{i}}$ of the last segment $s=S+1$ are transferred to the appropriate sub box. When a sub box crosses the periodic boundaries in the main system, we use the periodic image to transfer the information to the sub box. The full set of branch and end-points is indicated by $\left\{\mathbf{r}_{\mathbf{0}}, \mathbf{r}_{\mathbf{S}+\mathbf{1}}\right\}$.

The first step is to compute the segment statistical weights $G(\mathbf{r})$. All branch and end-points, however, already occupy their lattice site and hence no other segment can go there. Therefore we will impose that the segment statistical weight is zero at these sites:

$$
G(\mathbf{r})= \begin{cases}e^{-u(\mathrm{r})}, & \mathbf{r} \notin\left\{\mathbf{r}_{\mathbf{0}}, \mathbf{r}_{\mathbf{S}+\mathbf{1}}\right\} \\ 0 & \mathbf{r} \in\left\{\mathbf{r}_{\mathbf{0}}, \mathbf{r}_{\mathbf{S}+\mathbf{1}}\right\}\end{cases}
$$

With these segment statistical weights we can now calculate the end-point distribution functions. Two complementary end-point distributions are found recursively, one by a so-called forward and the other by a backward propagator. The forward procedure is initialized by setting $G_{i}\left(\mathbf{r}, 0 \mid \mathbf{r}_{\mathbf{0}}^{\mathbf{i}}, 0\right)=1$ when $\mathbf{r}=\mathbf{r}_{\mathbf{0}}^{\mathbf{i}}$ for spacer $i$ and 0 otherwise.

$$
G_{i}\left(\mathbf{r}, s \mid \mathbf{r}_{\mathbf{0}}^{\mathbf{i}}, 0\right)=G(\mathbf{r})\left\langle G_{i}\left(\mathbf{r}, s-1 \mid \mathbf{r}_{\mathbf{0}}^{\mathbf{i}}, 0\right)\right\rangle
$$

In Eqn 5.7 the angular brackets implement the averaged value over the neighbouring lattice sites. For a simple cubic lattice this gives:

$$
\begin{aligned}
\langle G(\mathbf{r})\rangle= & \langle G(X, Y, Z)\rangle \\
= & \frac{1}{6}((G(X+1, Y, Z)+G(X-1, Y, Z)+G(X, Y+1, Z)+ \\
& G(X, Y-1, Z)+G(X, Y, Z+1)+G(X, Y, Z-1))
\end{aligned}
$$

where it is understood that we kept in the notation only the spatial coordinate, that is, $G(\mathbf{r})$ is a shorthand for $G\left(\mathbf{r}, s \mid \mathbf{r}_{\mathbf{0}}, 0\right)$. The 'jump' to 
the last segment $s=S+1$ is given by

$$
G_{i}\left(\mathbf{r}, S+1 \mid \mathbf{r}_{\mathbf{0}}^{\mathbf{i}}, 0\right)= \begin{cases}\left\langle G_{i}\left(\mathbf{r}, S \mid \mathbf{r}_{\mathbf{0}}^{\mathbf{i}}, 0\right)\right\rangle, & \mathbf{r}=\mathbf{r}_{\mathbf{S}+\mathbf{1}}^{\mathbf{i}} \\ 0 & \mathbf{r} \neq \mathbf{r}_{\mathbf{S}+\mathbf{1}}^{\mathbf{i}}\end{cases}
$$

The backward propagator starts from the other end of the spacer and hence is started with $G_{i}\left(\mathbf{r}, S+1 \mid \mathbf{r}_{\mathbf{S}+\mathbf{1}}^{\mathbf{i}}, S+1\right)=1$ when $\mathbf{r}=\mathbf{r}_{\mathbf{S}+\mathbf{1}}^{\mathbf{i}}$ and zero otherwise. The calculation of the backwards propagator is continued with:

$$
G_{i}\left(\mathbf{r}, s \mid \mathbf{r}_{\mathbf{S}+\mathbf{1}}^{\mathbf{i}}, S+1\right)=G(\mathbf{r})\left\langle G_{i}\left(\mathbf{r}, s+1 \mid \mathbf{r}_{\mathbf{S}+\mathbf{1}}^{\mathbf{i}}, S+1\right)\right\rangle
$$

until at $s=0$

$$
G_{i}\left(\mathbf{r}, 0 \mid \mathbf{r}_{\mathbf{S}+\mathbf{1}}^{\mathbf{i}}, S+1\right)= \begin{cases}\left\langle G_{i}\left(\mathbf{r}, 0 \mid \mathbf{r}_{\mathbf{S}+\mathbf{1}}^{\mathbf{i}}, S+1\right)\right\rangle, & \mathbf{r}=\mathbf{r}_{\mathbf{0}}^{\mathbf{i}} \\ 0 & \mathbf{r} \neq \mathbf{r}_{\mathbf{0}}^{\mathbf{i}}\end{cases}
$$

The volume fraction $\varphi(\mathbf{r}, s)$ of segment $s$ in lattice site $r$ is proportional to the sum of the statistical weights of all the chain conformations that pass through lattice site $r$ with segment $s$. Each chain conformation going from segment 0 to segment $s$ can be combined with any of the chain conformations going from $S+1$ to $s$. The volume fraction distribution of segments $s=0, \cdots, S+1$ for the fragment $i$ is therefore given by the product of two end-point distribution functions, one computed starting from segment $s=0$ and the other by starting with segment number $S+1$, both ending with segment $s$ at the same coordinate $\mathbf{r}$,

$$
\varphi_{i}(\mathbf{r}, s)= \begin{cases}\frac{1}{q_{i}} \frac{G_{i}\left(\mathbf{r}, s \mid \mathbf{r}_{\mathbf{0}}^{\mathbf{i}}, \mathbf{0}\right) G_{i}\left(\mathbf{r}, s \mid \mathbf{r}_{\mathbf{S}+\mathbf{1}}^{\mathbf{i}}, \mathbf{S}+\mathbf{1}\right)}{G(\mathbf{r})}, & \mathbf{r} \notin\left\{\mathbf{r}_{\mathbf{0}}, \mathbf{r}_{\mathbf{S}+\mathbf{1}}\right\} \\ 0 & \mathbf{r} \in\left\{\mathbf{r}_{\mathbf{0}}, \mathbf{r}_{\mathbf{S}+\mathbf{1}}\right\}\end{cases}
$$

where $\mathbf{r}_{\mathbf{0}}^{\mathbf{i}}$ and $\mathbf{r}_{\mathbf{S}+\mathbf{1}}^{\mathbf{i}}$ are the locations of the two ends of spacer $i$. In Eqn 5.12 the division by $G(\mathbf{r})$ is necessary because the weight for segment $s$ is accounted for in both end-point distribution functions that are in the numerator. Obviously, there can not be any other segment in the specified branch points, therefore $\varphi_{i}(\mathbf{r}, s)=0$ when $\mathbf{r} \in\left\{\mathbf{r}_{\mathbf{0}}, \mathbf{r}_{\mathbf{S}+\mathbf{1}}\right\}$. The normalisation by $\frac{1}{q_{i}}$ ensures that there is exactly one segment $s$ of molecule fragment $i$ in the sub box. Here $q_{i}$ is the chain partition function, which is the combined statistical weight of all possible and 
allowed conformations of the chain fragment. It is given by:

$$
q_{i}=G\left(\mathbf{r}_{\mathbf{S}+\mathbf{1}}^{\mathbf{i}}, S+1 \mid \mathbf{r}_{\mathbf{0}}^{\mathbf{i}}, 0\right)
$$

The partition function for the solvent is:

$$
q_{O}=\sum_{\mathbf{r}} G(\mathbf{r})
$$

The overall volume fraction distribution of the dendrimer is found by adding up the segment distributions of all the spacers:

$$
\varphi(\mathbf{r})=\sum_{i} \sum_{s=1}^{S} \varphi_{i}(s, \mathbf{r})
$$

when $\mathbf{r} \notin\left\{\mathbf{r}_{\mathbf{0}}, \mathbf{r}_{\mathbf{S}+\mathbf{1}}\right\}$ and $\varphi(\mathbf{r})=1$ otherwise. Again, the segment potentials are adjusted iteratively until the volume fractions of the solvent and the dendrimer add up to unity at each coordinate in the main box. This SCF solution is obtained with at least 5 significant digits.

At this point we have all the information we need to determine the free energy $F$.

$$
F=-\ln q_{O}-\sum_{i} \ln q_{i}-\sum_{\mathbf{r} \notin\left\{\mathbf{r}_{\mathbf{0}}, \mathbf{r}_{\mathbf{S}+\mathbf{1}}\right\}} u(\mathbf{r})
$$

This free energy is then used as the classical 'energy' in the MC formalism. This means that the Metropolis acceptance is taken with the probability $p=e^{-\Delta F}$ when $\Delta F>0$ and $p=1$ otherwise. Here $\Delta F=F_{\text {new }}-F_{\text {old }}$. For the Monte Carlo trial step a translational move is implemented. A known feature of the simple cubic lattice is that for a specified start and stop site, one either needs an odd or an even number of steps. This implies that if the starting coordinate is shifted by one site, the walks that connect these points go from having an even to an odd number of steps or vice versa. As all spacers have a fixed length $S$, the one-step trials are forbidden. Therefore it is necessary to move any point an even number of steps. In the translational trial step a branch point or an end-point is selected randomly and its position is changed, by one lattice site in a random direction, twice. 
The number of branch/end-points that were moved within one trial step was optimized during the equilibration run, such that the acceptance probability times the number of branch/end-points moved was maximum. During data gathering the number of branch/end-points that were moved was fixed. We first did an equilibration run of 10000 Monte Carlo steps, which was followed by data gathering for 90000 steps. Such simulations ran many days and therefore longer simulations were not feasible for the largest dendrimers. Optimisation of the calculation efficiency may improve the speed significantly and in that case better averages can be obtained.

\section{Cell Model}

In the cell model ${ }^{54 / 59}$ we assume that the system is spherically symmetric. This is implemented by using a one-gradient spherical coordinate system. In such a coordinate system lattice sites lie in concentric layers of thickness $l$ which are numbered $r=1, \cdots, m$. There is just one value of the segment potential and the volume fractions per layer. This greatly reduces the computational cost.

In the spherical geometry the number of lattice sites in each layer grows roughly quadratically: $L(r)=V(r)-V(r-1) \propto r^{2}$, with the dimensionless volume $V(r)=\frac{4}{3} \pi r^{3}$. The root segment of the dendrimer is pinned to $r=1$. The upper limit $r=m$ is larger than the longest path in the dendrimer $g(S+1)$, so the polymer density at the upper bound is zero. Just as for the simple cubic lattice we assume that each lattice site has $Z=6$ nearest neighbours. One in the layer above, one in the layer below and four in the same layer. Because the outer surface area is larger than the inner surface area the a priori probability to go outward is larger than the probability to go inward. We therefore use :

$$
\begin{aligned}
\lambda_{+}(r) & =\frac{4 \pi r^{2}}{6 L(r)} \\
\lambda_{-}(r) & =\frac{4 \pi(r-1)^{2}}{6 L(r)} \\
\lambda_{0}(r) & =1-\lambda_{+}(r)-\lambda_{-}(r)
\end{aligned}
$$

Here, $\lambda_{+}(r)$ is the a priori probability to go from $r$ to $r+1, \lambda_{-}(r)$ the 
probability to go from $r$ to $r-1$ and $\lambda_{0}(r)$ the probability to go to a lattice site in the same layer $r$.

These probabilities are used in the propagators. In the spherical coordinate system the side fraction is given by:

$$
\langle G(r)\rangle=\lambda_{-}(r) G(r-1)+\lambda_{0}(r) G(r)+\lambda_{+}(r) G(r+1)
$$

wherein $G(r)$ is a shorthand for the appropriate end-point distribution functions. Symmetric dendrimers have the property that all paths from the root segment to any of its ends are equally long. Let's number the segments of one such path. From the zeroth segment $(s=0)$ pinned at $(r=1)$ to segments in the first $j=1$ generation, $s=1, \cdots, S+1$, for the $j=2$ generation we have numbers $s=S+2, \cdots, 2(S+1)$, etcetera, until at the end of generation $j=g$ we find ranking numbers $s=(g-1)(S+1)+1, \cdots, g(S+1)$. The free ends thus have the ranking number $s=g(S+1)$.

Typically the volume fraction distribution is computed starting with the backward propagators at one of the free ends of the dendrimer. As this end is not restricted in space, the end-point distribution function is integrated over all possible starting positions $G(r, s \mid g(S+1))=$ $\sum_{r_{g(S+1)}} G\left(r, s \mid r_{g(S+1)}, g(S+1)\right)$. The backwards propagator is initiated by $G(r, g(S+1) \mid g(S+1))=G(r)$ for all $r$, and we then propagate towards the root segment. For segments of generation $j=g$

$$
G_{g}(r, s \mid g(S+1))=G(r)\left\langle G_{g}(r, s+1 \mid g(S+1))\right\rangle
$$

that is, for $s=g(S+1)-1, \cdots,(g-1)(S+1)+1$. Here, we have added a sub-index to $G$ to point to the generation the end-point distribution 'belongs' to. Going inward $f-1$ chains will merge at the branch point and we re-initiate:

$$
\begin{aligned}
& G_{g-1}(r,(g-1)(S+1) \mid g(S+1))= \\
& G(r)\left\langle G_{g}(r,(g-1)(S+1)+1 \mid g(S+1))\right\rangle^{f-1}
\end{aligned}
$$

With this we proceed to generation $j=g-1$

$$
G_{g-1}(r, s \mid g(S+1))=G(r)\left\langle G_{g-1}(r, s+1 \mid g(S+1))\right\rangle
$$


for $s=(g-1)(S+1)-1, \cdots,(g-2)(S+1)+1$. Then again we need to re-initiate, as other (equivalent) chain fragments join the walk and we proceed with $j=g-2$, etcetera, until we finished generation $j=1$ and finally reach $s=0$. For this segment we need to use $G_{0}(r, 0 \mid g(S+1))=$ $G(r)\left\langle G_{1}(r, 1 \mid g(S+1))\right\rangle^{f}$. As we have pinned the root segment in the origin $(r=1), G_{0}(r, 0 \mid g(S+1))$ is set to zero for $r \neq 1$. At this point we can evaluate the partition function $q_{D}=G_{0}(1,0 \mid g(S+1)) L(1)$. For the solvent the partition function is $q_{O}=\sum_{r} L(r) G(r)$.

When $r=1$, the forward propagator starts with: $G_{1}(r, 0 \mid 1,0)=$ $G(r)\left\langle G_{1}(r, 1 \mid g(S+1))\right\rangle^{f-1}$, otherwise it is initialized with zero. Next, we propagate through the first generation $j=1$ :

$$
G_{1}(r, s \mid 1,0)=G(r)\left\langle G_{1}(r, s-1 \mid 1,0)\right\rangle
$$

For $s=1, \cdots, S+1$. Before we can continue with $j=2$ we have to 'add-on' the $f-2$ branches of $G_{2}(r, S+2 \mid g(S+1))$. Hence, the end-point distributions are re-initiated:

$$
\left.\left.G_{2}(r, S+1 \mid 1,0)=G_{1}(r, S+1 \mid 1,0)\left\langle G_{2}(r, S+2)\right| g(S+1)\right)\right\rangle^{f-2}
$$

and we can continue towards the next branch point:

$$
G_{2}(r, s \mid 1,0)=G(r)\left\langle G_{2}(r, s-1 \mid 1,0)\right\rangle
$$

for $s=S+2, \cdots, 2(S+1)$ and we repeat this procedure for each generation until we arrive at the last generation $j=g$ at the free end with $G_{g}(r, g(S+1) \mid 1,0)$.

Combining the forward and backward end-point distribution functions gives the volume fraction profiles:

$$
\varphi_{j}(r, s)=\frac{f(f-1)^{j-1}}{q_{D}} \frac{G_{j}(r, s \mid 1,0) G_{j}(r, s \mid g(S+1))}{G(r)}
$$

The distribution of segment $s=0$ is zero everywhere except in layer $r=1$ where it has the value $\varphi(1,0)=1 / L(1)$. The overall volume fraction distribution for the dendrimer in the cell model is found by summing over all segment ranks $s$ from the root to the ends. The index $j$ can then be dropped. We can also collect the segments that belong 
to a part of the molecule, e.g, for a particular sub-generation $j$ or a branch point (the last segment of a sub-generation). We refer to the literature $\sqrt{26 \mid 105}$ for more details about dendrimers in the SF-SCF cell model.

\section{Data Analysis}

We determined the overall density distribution relative to both the root segment and the centre of mass, and the distribution of the branch and end-points relative to the root segment. For the FJC model we could also evaluate the distribution of the branch- and end-points relative to the centre of mass. The classical way to compare different models is to compute the radius of $R_{g}$ which is a measure for the size of the dendrimers. This quantity is defined as

$$
R_{g}=\left\langle\left(\frac{1}{N} \sum_{h=1}^{N}\left(\mathbf{r}_{\mathbf{h}}-<\mathbf{r}>\right)^{\mathbf{2}}\right)^{\frac{1}{2}}\right\rangle
$$

Here $N$ is the total number of monomers/particles in the system, $<\mathbf{r}>$ the average position/centre of mass and $r_{h}$ is the coordinate of the particle $h$.

In the cell model the dendrimers are by definition radially symmetric. In practice it is known that deviations from the spherical shape are important. Following the definition of Rudnick et. al. $\stackrel{106}{ }$ we computed the asphericity for the MC-FJC and MC-SCF methods. This measure indicates how different the size of the object is in the three perpendicular directions. One of the axis points in the direction in which the object is the largest and one in the direction in which the object is smallest. The third axis is perpendicular to these two. The first step to determine the asphericity is to calculate the gyration tensor $T$.

$$
T_{i j}=\frac{1}{N} \sum_{h=1}^{N}\left(\mathbf{r}_{\mathbf{h i}}-<\mathbf{r}_{\mathbf{i}}>\right)\left(\mathbf{r}_{\mathbf{h j}}-<\mathbf{r}_{\mathbf{j}}>\right)
$$

Here $i$ and $j$ are the spatial directions and $r_{h i}$ thus indicates the position of particle $h$ on the $i$ axis. Subsequently, the eigenvalues $\lambda$ of the matrix $T$ are determined. The magnitude of the eigenvalues is a mea- 

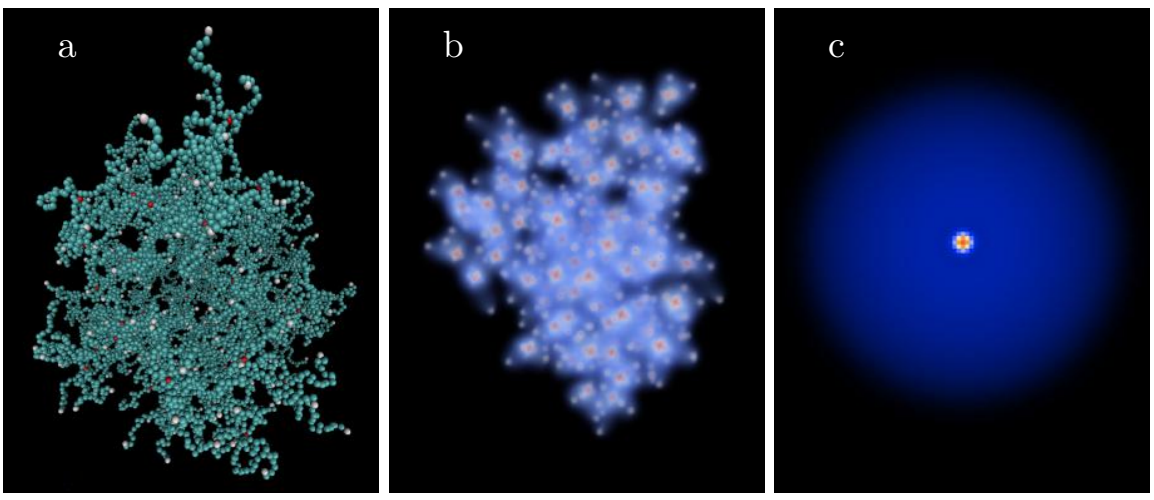

Figure 5.1: Snapshots of a dendrimer with $f=5, g=4, S=20$ : (a) the MC-FJC model. The branch points are coloured red and the end-points white, (b) the MC-SCF model and (c) the cell model. For (b) and (c) the segment density decreases going from orange, through white to blue and black. (The scale differs between the images)

sure for how extended the object is in each extremal direction. Next, the asphericity $A$ is calculated as:

$$
A=\frac{\sum_{i=1}^{d-1} \sum_{j>i}^{d}\left(\lambda_{i}-\lambda_{j}\right)^{2}}{(d-1)\left(\sum_{i=1}^{d} \lambda_{i}\right)^{2}}
$$

Here $d$ is the number of spatial dimensions.

\section{Results and Discussion}

In Figure 5.1 we show results for a dendrimer with $f=5, g=4, S=$ 20. The first image (a) is a snapshot of the MC-FJC model. For the MC-FJC model the branch points including the root segment, which is just visible in the centre, are coloured red. The second image (b) is a snapshot of the MC-SCF model. The branch and end-points are visible as orange crosses and white dots with an orange centre, respectively. They are easily found as the segment density around these points is high. For illustrative purposes we present the cell model (c), in an unusual way, as a density plot. The density drops from high density 

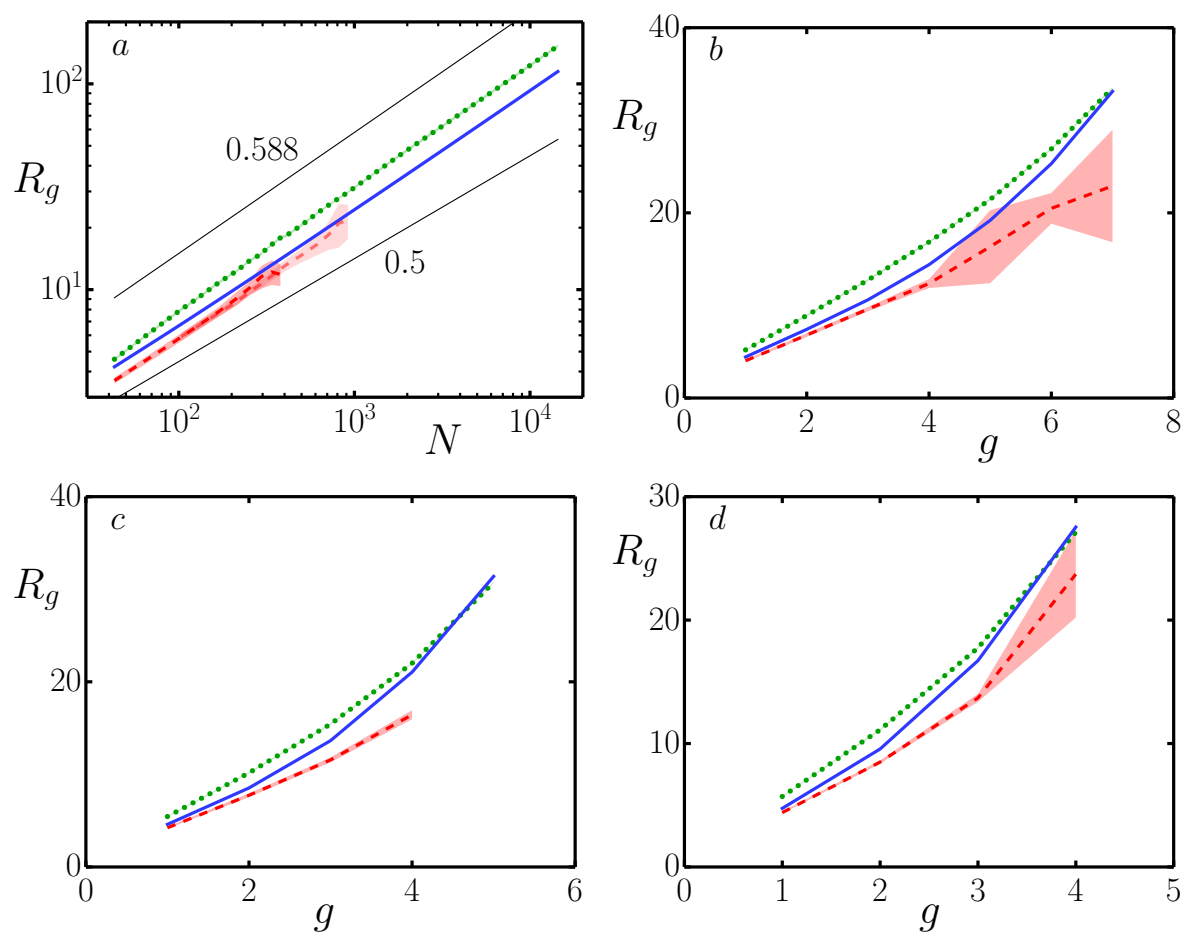

Figure 5.2: Radius of gyration in units $l$ as a function of: a) the overall degree of polymerisation $N$ for linear chains $(f=2)$ and b) $f=3$, c) $f=4$, d) $f=5$ as a function of the generation number $g$. Cell model (-), MC-SCF $S=20(---)$, MC-SCF $S=50(---)$ and the MC-FJC (" " " The lighter areas indicate the $99 \%$ confidence interval.

in the core to low density in the corona. There are many similarities between the first two images. Both dendrimers are roughly spherical, but show significant density fluctuations in azimuthal direction. Such fluctuations are not present in cell model (image c) due to the mean field approximation. The spacers are distributed over all the possible conformations and are visible as the bluish aura around the branch and end-points. Only image (a) is a true single conformation snapshot because all segments have a specific position.

In Figure $5.2 \mathrm{a}$ the radius of gyration $R_{g}$ of linear chains is shown for the different models. The linear chain is a special dendrimer, namely 
with $f=2$ and it is interesting to consider this case first. The result for a linear chain is the well known $R_{g} \propto N^{\alpha}$, with $\alpha$ going towards 0.588 in the high chain length limit. $\stackrel{41 / 42}{ }$ The MC-FJC approaches this value from above, as in the short chain limit of just two beads it behaves as a rigid rod. The cell model on the other hand approaches from below. Because it allows backfolding, the cell model can behave as a Gaussian coil for small chain lengths and it therefore approaches from 0.5. For the MC-SCF model $\alpha$ is about 0.59 and numerical inaccuracies do not allow us to pinpoint whether it approaches from above or below. No significant difference between middle block lengths of $S=20$ and $S=50$ was observed, although the large confidence intervals could still hide small differences between the two.

It is interesting to point out that the value for the radius of gyration $R_{g}$ for the MC-SCF model is the smallest of the three models and that MC-FJC gives the largest value. Due to the fact that for the spacers backfolding is allowed, in both the cell model and the MC-SCF model, they give a smaller $R_{g}$ than the MC-FJC model. The cell model also overestimates the long range steric hindrance and therefore the chain is bigger than in the MC-SCF model.

In Figures $5.2 \mathrm{~b}$-d the radius of gyration $R_{g}$ is shown for dendrimers with $f=3, f=4$ and $f=5$, respectively, as a function of the generation number $g$. Let's focus first on the difference between the least accurate (cell model) and the most accurate (MC-FJC) model. For low generation numbers $g$ the cell model (solid line) underestimates the size significantly. As the number of generations increases the cell model catches up with the MC-FJC model and for $f=4, g=5$ and $f=5, g=4$ the radius of gyration exceeds that of the MC-FJC model. As we mentioned before this more rapid growth of the radius of gyration is likely caused by the overestimation of the steric repulsion between the branches of the dendrimer. In a real polymer the segments are next to each other and their excluded volumes will overlap. In the cell model the segments are distributed homogeneously within the lattice layers and there is little overlap of the excluded volumes, hence the stronger stretching. For a small number of generations the underestimation of the short-range excluded volume causes the too small size.

At first sight it may seem that the hybrid MC-SCF model is even worse, as it consistently gives a too small size for the dendrimer. The 
shapes of the curves are however very similar to that of the MC-FJC model. If the radius of gyration of the MC-SCF model is multiplied with about 1.31 for $S=20$ and 1.37 for $S=50$ the curves overlap. The scaling behaviour of the MC-SCF model should therefore be very similar to the FJC model although the relatively large confidence interval of the MC-SCF model leaves some room for deviations.

In the literature ${ }^{98 \mid 99}$ there exists a prediction for the power-law dependence of the size of dendrimers, $R_{g} \propto(S g)^{\frac{2}{5}} N^{\frac{1}{5}}$. This equation gives a fairly good fit, although we typically find that it slightly underestimates how much the dendrimers swell as the number of generations is increased. Using slightly larger exponents of 0.41 instead of 0.40 and 0.22 instead of 0.20 gives a better fit for $f>2$. For the limiting case of linear chains these exponents are however not a good fit. We found an alternative equation:

$$
R_{g} \approx 0.72(g S)^{0.608}\left(1+0.123(f-2)^{0.70}\right)^{g}
$$

which is a bit better at describing the $R_{g}$ as a function of $f, g$ and $S$ when linear chains are also considered. The numbers with digits behind the comma were determined by fitting the equation to the data. We started with a power law $(g S)^{\alpha}$ as is usual for linear polymers and subsequently added a correction factor to account for the extra swelling due to the other dendrimer arms. There is no further theoretical basis for this equation. As we did not vary the interaction parameters between the beads we did not incorporate these effects.

Let us next focus on the average distance of the end segments from the central segment. Even though it is expected that this quantity has similar information as the radius of gyration, we present a selection for these distributions in Figure 5.3. As in the previous Figure we focus in panel a) on the $N$-dependence for $f=2$ (linear chains) and in b) on the dependence on the generation number $g$, for $f=3$ and note that for $f=4,5$ similar trends were obtained.

For the end-point distance one would again expect a power-law dependence. To a reasonable approximation this indeed happens, albeit that in all cases we see a somewhat larger deviation from the expected limiting power law value of 0.588 than for the radius of gyration (cf fig. 5.2 ), especially for the MC-SCF model for which the exponent is about 

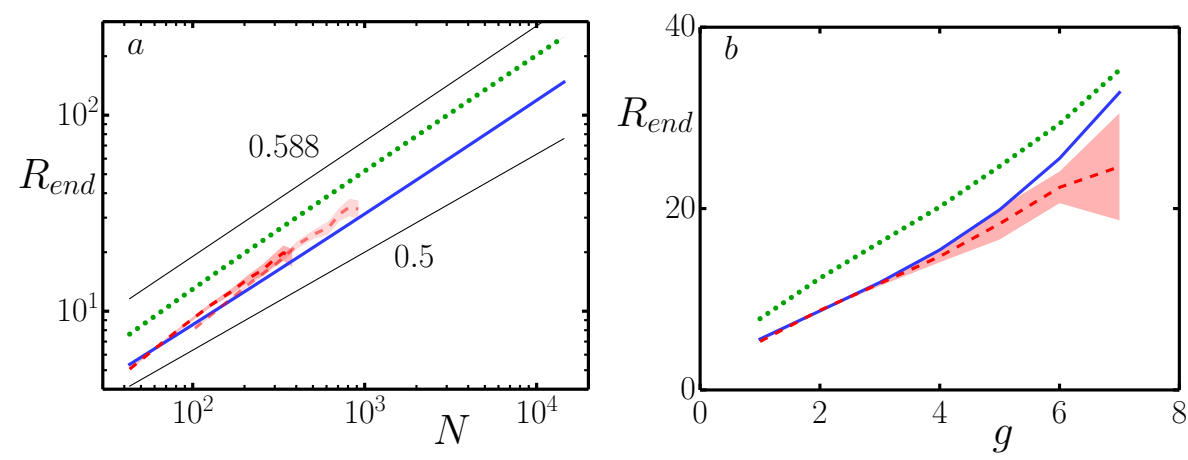

Figure 5.3: The average end segment distance relative to the central segment $R_{\text {end }}$ in units $l$ for: a) linear chains $(f=2)$ as a function of $N$. b) for $f=3$ as a function of the generation number $g$. Cell model (-), MC-SCF $S=20(---)$, MC-SCF $S=50(---)$ and the MC-FJC (" * "). The lighter areas indicate the $99 \%$ confidence interval.

0.65. These deviations are again attributed to the finite chain lengths. Apparently we should view the MC-SCF chain as a bead spring model, with each branch and end-point as a bead. These beads would interact through soft potentials generated by the spacers. As the number of beads in MC-SCF is very small we see large deviations from the limiting values. We also see such large deviations for the end-point distances of the MC-FJC model when very short spacers are used. Just as for the MC-SCF model, these differences are larger for the end-point distances than for the radius of gyration.

In Figure 5.3 we show results for both $S=20$ and $S=50$ for the MC-SCF model. The larger the value of $S$, the fewer MC-beads $(N / S)$ we have in the simulation. We noticed that the average position of the end-points differs between $S=20$ and $S=50$. The curve for $S=50$ starts below the curve of $S=20$ and this difference diminishes with increasing $N$. This implies that the corresponding power-law coefficient is larger for $S=50$, which is expected if the MC-SCF chains are viewed as a bead spring model. Recalling that the radius of gyration was the same for these two cases, it implies that the radial density distribution should be different.

In Figure 5.4 we present the radial density profile for two linear 


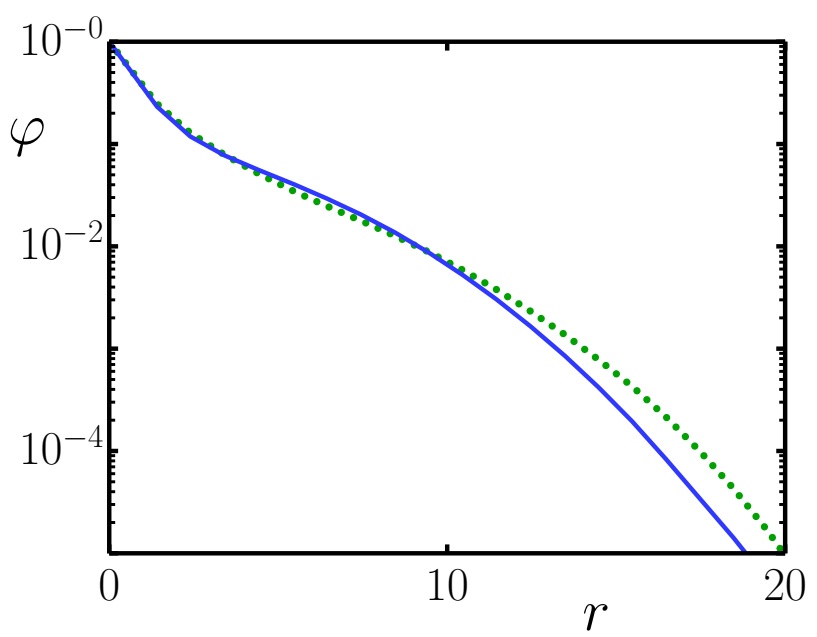

Figure 5.4: The average radial density profile relative to the central segment as found by the MC-SCF model for $f=2$. The two cases have the same value of $N: S=62, g=1(-), S=20, g=3(" \cdots)$.

chains with the same number of segments $N=63$ modelled by the MC-SCF hybrid. The two results differ with respect to the number of MC-beads that were implemented. Close inspection of Figure 5.4 shows that the curves for $S=20, g=3$ and $S=62, g=1$ cross each other twice. At very short and large distances $r$ from the central segment the volume fraction $\varphi$ for $S=20, g=3$ is higher than for $S=62, g=1$. At intermediate distances the opposite is true. These two trends have the effect that $R_{g}$ is approximately the same for both, but as the end-segments are further away from the center, the largest end-point position is found for $S=20, g=3$.

A possible reason for this difference between $S=20$ and $S=62$ is that for $S=62$ it costs less entropy to go a few steps in the 'wrong' direction. The blobs of density around the branch points are therefore larger for $S=62$ than for $S=20$. In the centre of the blob the behaviour will be similar to the cell model. i.e. the density of the polymer chain is still smeared around the branch point, because there are still a fair number of conformations who move away from the next 
branch point in the first few steps on the lattice. Similar to the cell model the repulsion between different branches around the branch point is therefore over estimated. The area for which this effect takes places is larger for $S=62$ and therefore the spacers are stretched more near the central branch point which results in a faster decrease in density near the central segment. Near the end-point there is only one chain and this effect is much weaker. The longer spacer 62 can spread itself over a larger area and therefore the repulsion between segments next to each other in the spacer is less. The result is that the chain is less stretched near the ends and the average end-point distance is therefore smaller.

The average end-point distance for $f=3$ is shown in Figure $5.3 \mathrm{~b}$. In general the trend for the end-point distance is the same as for the radius of gyration. Again we see that the full excluded volume MCFJC result gives the largest dendrimer size. For low values of $g$ the cell model and the MC-SCF do not differ much. For large $g$ the cell model goes towards the MC-FJC result and it is expected that it will overtake this for sufficiently large $g$. The size of the dendrimer in the MC-SCF model stays more systematically below that of the MC-FJC model, suggesting once again that there is just a different prefactor. Close inspection reveals that a similar shift as reported above for the $R_{g}$ results does not give the same quality of overlap of curves in this case.

In Figure 5.5 the radial density profiles, relative to the central segment, are shown for a functionality $f=2,3,4,5$ with $S=50$ for $f=2$ and $S=20$ for $f>2$, and respective generations $g=9,7,4,4$. For the linear polymers (panel a) the density drops monotonically and the inner region can be approximated by a power law $\varphi(r) \propto r^{\beta}$. For all three models $\beta \approx-\frac{4}{3}$, with a slightly larger value for the MC-FJC model and a slightly smaller value for the cell model. This is consistent with the work of de Gennes $\frac{40}{4}$, who also predicted a value of $-\frac{4}{3}$. For the MC-SCF model there seems to be a slight kink in the early part of the curve which may be related to a change in behaviour going from length scales less than the inter node distance to length scales larger than the inter node distance. We should be careful not to over interpret these numbers because we definitely need better statistics to find accurate values for the density in and around the center. For MC-FJC 
Results and Discussion
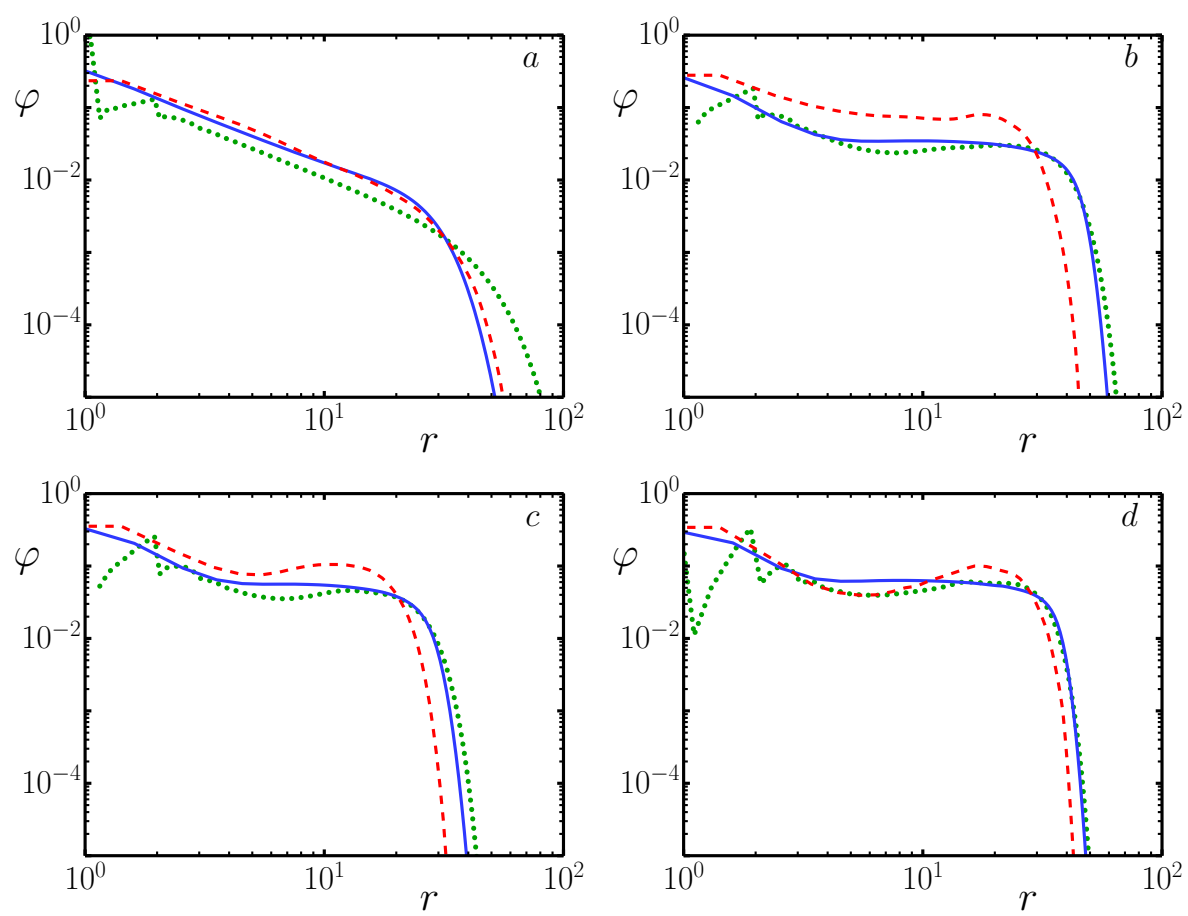

Figure 5.5: Radial density profiles relative to the central segment for a) $f=2, g=9, S=50$, b) $f=3, g=7, S=20$, c) $f=4, g=4, S=20$, d) $f=5, g=4, S=20$. The cell model (-), MC-SCF (- - -), and the MC-FJC ( " " ").

an irregular distribution is found in the very center. Near the centre the branches are strongly stretched, the second segment from the centre therefore is mostly in the positions furthest from the centre. This results in a peak at $r=2$. These effects are frequently seen in simulations when excluded volume effects are truly accounted for and local densities are high.

Inspection of Figure $5.5 \mathrm{~b}-\mathrm{d}$, shows that for both the MC-SCF and the MC-FJC model there exists a clear dip in the density profile of the dendrimers. There is only a marginal dip of approximately $3 \%$ for the cell model. This is in line with the results of Klein Wolterink et al. $\frac{105}{}$, who did not observe a dip for neutral dendrimers in a cell 
model. According to our simulations and in line with other dendrimer simulations $\frac{101 / 107 / 108}{}$, a true dip in the radial density only occurs for systems with strong steric hindrance between the spacers, i.e. for $f=$ $3, g>5, f=4, g>3$ and $f=5, g>2$. The relative depth of this dip increases as the number of spacers per branch point $f$ or the number of generations $g$ is increased. The largest dip depth, for the MC-FJC model, is for $f=5$ and $g=4$ where the density is only $65 \%$ of the maximum further out. As mentioned, the results for the MC-SCF model are also consistent with a non-monotonic density profile, but numerical uncertainties in the local density prevent us from quoting a numerical value for the depth. Computer simulations by Chen et al. .100 who used a similar MC-FJC model with similar trial moves did not report a dip in the radial density profiles. Because these authors used slightly smaller beads than our FJC model and their results were noisier than our curves, they may have missed the dip in density.

Let us next consider a pair of key structural features inside the dendrimer. The first of these is the end-point distribution. Naïvely one could expect that the end-points are solely distributed at the outside of the dendrimer, but this view has been rejected and it is commonly understood that the ends are distributed throughout the dendrimers. Nevertheless it is interesting to see which distribution our models predict for the dendrimer. The other quantity is the distribution of the first branch points. These distributions are shown in Figure 5.6. Going from panel a) to d) the number of spacers per branch point $f=2,3,4,5$ increases, while the number of generations $g=9,7,4,4$ decreases, and all spacer lengths $S=20$. Going from a to d the amount of crowding of segments inside the dendrimers increases, with roughly the same amount of crowding for $\mathrm{b}$ and $\mathrm{c}$.

In Figure 5.6 the end-point distributions are plotted in light colours. Obviously, the end-points can reach higher $r$ values than the first branch point (whose distribution is given in the darker curves). Just like Rud et al. ${ }^{26}$, we find that all end-point distributions show a broad maximum and also give a finite value inside the central region of the dendrimer.

In comparison going from the linear chain (panel a) to the dendrimers with increasing crowding b) to d) we notice that the end distributions become more alike between the models. From the perspective of the chain ends there is no indication that the SCF method is failing 

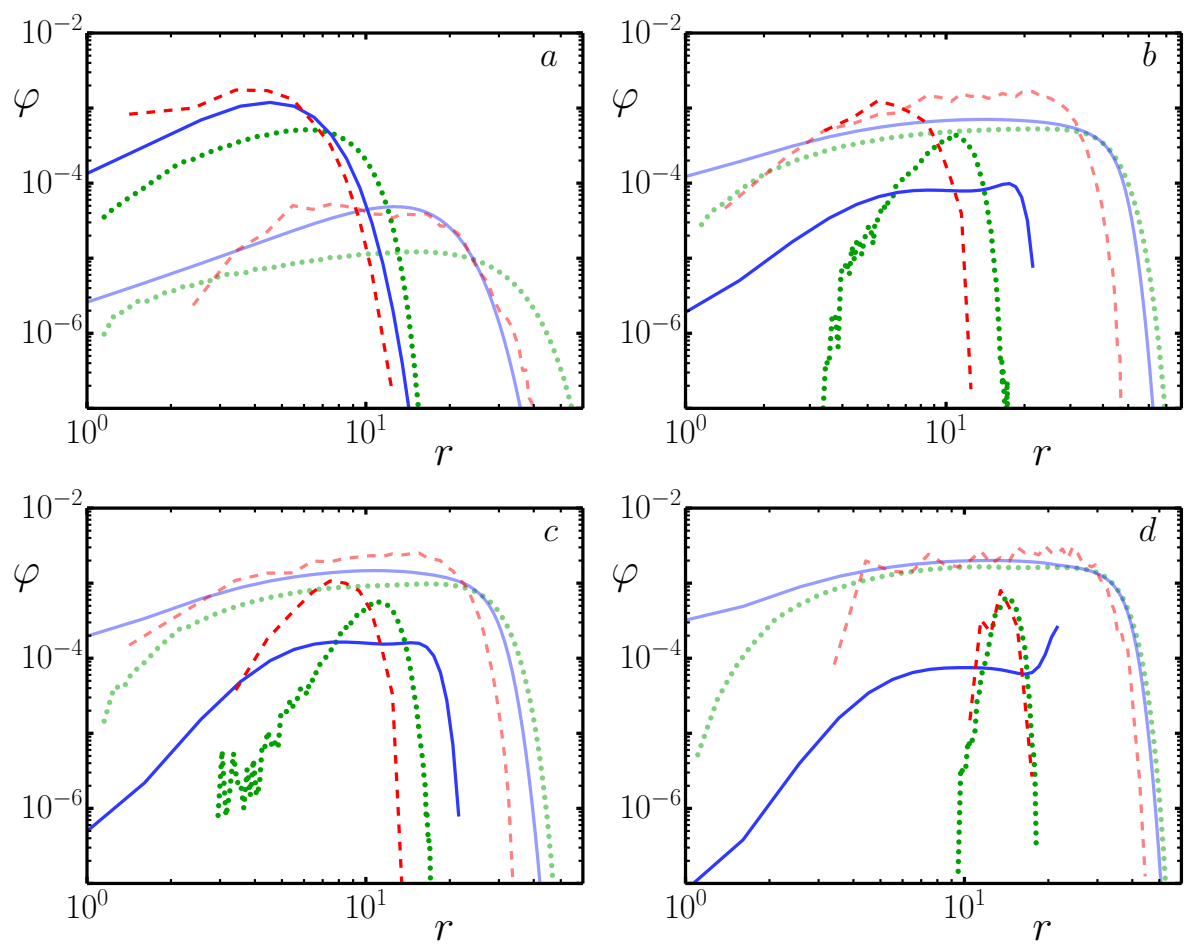

Figure 5.6: The volume fractions of the first branch point(dark) and end-points(lighter), relative to the position of the central segment, for a) $f=2, g=9, S=20, \mathrm{~b}) f=3, g=7, S=20$, c) $f=4, g=4, S=20$, d) $f=5, g=4, S=20$. cell model (-), MC-SCF (-- -), and the MC-FJC ( $\because \cdots)$.

in any way in the high crowding limit. The only difference between the cell model and the MC-FJC simulations is the depression of the endpoint distribution at the very centre of the chain. One possible cause is that in the Monte Carlo models the central segment is placed exactly in the origin. In the cell model the central segment is however placed in the first layer around the origin. The central segment is therefore half a lattice site away from the origin and the density in the centre is thus lower. Another explanation is that the last generation of branch points is on average further from the centre for the Monte Carlo models than 
for the cell model. The chains thus have to stretch more to reach the centre for the Monte Carlo models.

The results for the distribution of the first off-center branch point are much more interesting. Inspection of the darkly coloured curves in Figure 5.6 shows that the profiles resemble each other reasonably well for the linear chain, but deviate progressively more as the molecular crowding increases. There is just one trend that is the same for al models, namely, the outward shift of the branch points with increasing crowding. With the cell model (solid blue lines), we notice an initial increase in the width of the profiles and for stronger crowding (large $f$ and $g$-values) the distribution becomes clearly bimodal. A similar bimodal branch distribution has been observed previously by both Polotsky et al. ${ }^{109}$ with a SF-SCF model of brushes of dendrimer arms with $g=2,3,4, S=100$ and $f=2,3,4,5,8$ and Rud et al. ${ }^{26}$ who did a study on dendrimer arms attached to surfaces of various curvature, again using the cell model. The results of Klein Wolterink et al. 105 also show a peak near maximum extension for the distribution of the first branch points. Due to the way they plotted the data it is however not clear whether a second peak is also present. Merlitz et al. $\stackrel{110}{ }$ used a Langevin dynamics simulation to study brushes made of dendrimer arms of a dendrimer with $S=50, f=4$ and $g=2$. They observed two peaks in the density distribution of the end-points and from this they also concluded there were two populations.

The common interpretation of this bimodal distribution for brushes is that there are two conformation populations for the dendrimers. The first population has a weakly stretched first spacer, and its segments fill up the density near the surface. The second population has a fully stretched first spacer and these conformations provide the segment density at the outside of the brush. In our case we see similar effects inside one dendrimer. The interpretation therefore is that the different arms connected to the central branch point have different conformations. The arms with weakly stretched first spacer are mostly in the interior of the dendrimer, the arms with a fully stretched first spacer make up most of the outside of the dendrimer.

This cell model result should be compared to predictions of the MC-FJC model. With increasing crowding (higher $f$ and $g$ values, i.e. going in Figure 5.6 from panels $b$ to $d$ ) the profile of the first 
branch point sharpens while it shifts outwards. Unlike the cell model the position of the maximum does not reach the fully extended value. At very large crowding (panel d) the maximum is positioned in between the two maxima found by the cell model, not far from the local minimum. In stark contrast to the cell model, there is thus no tendency to go to a bimodal distribution with increased crowding.

The MC-SCF hybrid follows the result of the MC-FJC model. Also with this method it is found that the distribution of the first generation of branch points sharpens and moves to higher $r$ values upon an increase of $f$ and $g$. Inspection of panel $d$ shows that MC-SCF and the full MC model give virtually the same profile for the branch points. While at intermediate crowding (panels $\mathrm{b}$ and $\mathrm{c}$ ) the distribution of the branch points for the MC-SCF has a similar shape as the MC-FJC model, although the spacers are less stretched. Within the MC-SCF model there is also no trend towards bimodality of the branch point distribution.

Interestingly, at intermediate crowding, both MC-models gradually develop a so-called dead zone, i.e. a region near the centre where no branch points are found. Such a dead zone is not present in the cell model. In MC-FJC and MC-SCF the dead zone widens with increasing crowding. We can only conclude that in neither there are indications for two populations of dendritic arms in a given dendrimer.

There are several effects that together may have caused the deviating behaviour for the SCF cell model. As we have stated before the steric repulsion with other branches is overestimated in the cell model. The force to stretch the spacers will therefore be a bit stronger in the cell model. This may have triggered the 'transition' towards two populations. As the short-range excluded volume is underestimated by SCF, the fragment that is 'retracted' can do so more easily than in reality and this might have stabilized the two-population state.

Yet another reason is that in the cell model it is possible to retract, for example, 0.5 dendrimer arms, while in the more realistic MC-FJC and MC-SCF models only whole arms can be retracted. Of course, we cannot exclude the possibility that for higher functionalities and/or a larger number of generations, one of the arms folds inwards completely and effectively fills the dip in density as we have seen in Figure 5.5 . In that case the MC-SCF and MC-FJC models may thus also develop two populations. 

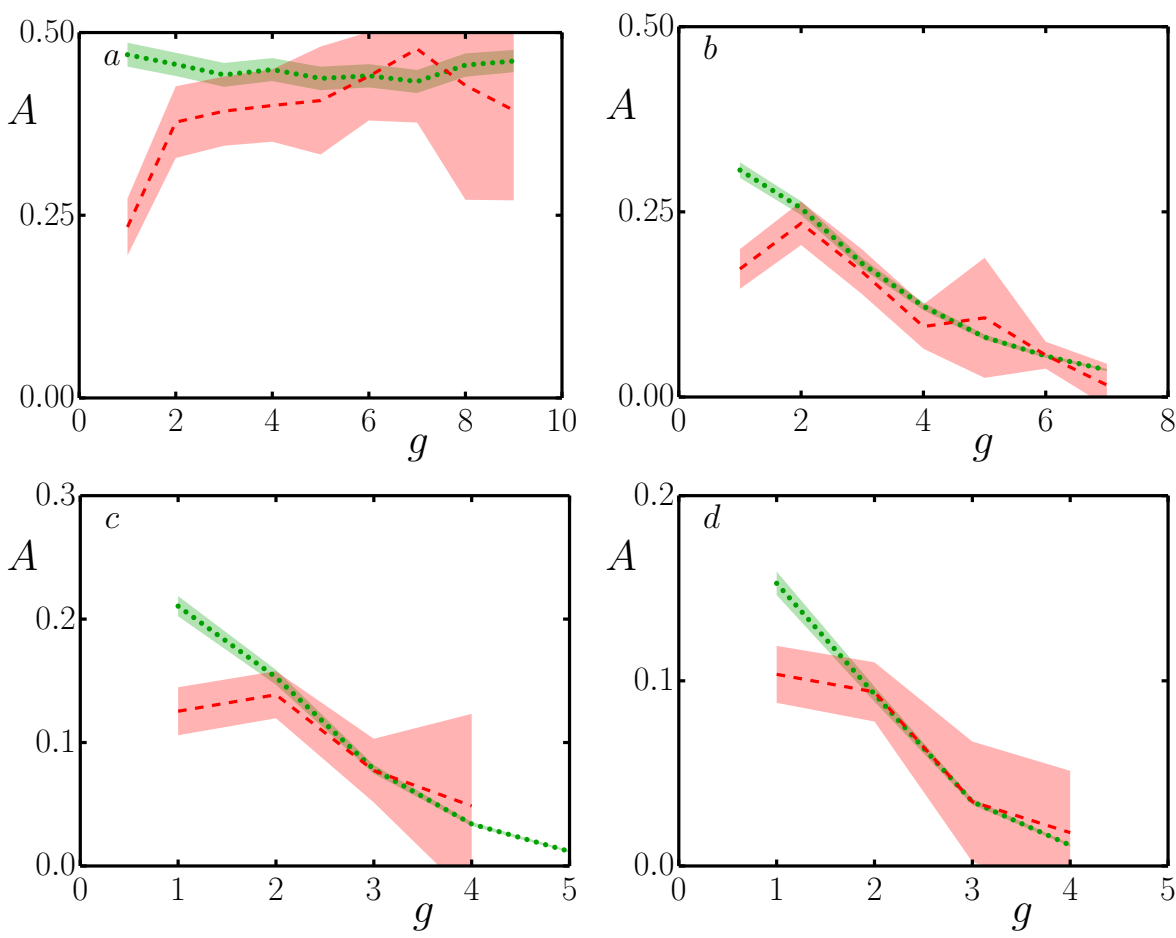

Figure 5.7: The asphericity for a) $f=2, S=20$, b) $f=3, S=20$, c) $f=4, S=20$, d) $f=5, S=20$. MC-SCF (-- -), and the MC-FJC $(\because \cdots)$ The lighter areas indicate the $99 \%$ confidence interval.

Interestingly, for weakly dissociating polyelectrolyte stars Uhlik et al. $\stackrel{111}{ }$ have also observed a wide and bimodal distribution of end-points for the SF-SCF model. Monte Carlo simulations however showed a much narrower unimodal distribution. $\stackrel{111}{111}$ For a poor solvent they however did find a bimodal distribution. $\frac{112}{112}$ They ascribed this difference to the inaccurate way in which the SCF method accounts for intramolecular excluded volume, but did not go into detail. Arguably, the conservative advise therefore is to approach bimodal distributions found by SCF theory with caution, especially when there is not a clear mechanism that can support bimodality (e.g. in 'gas'-'liquid' equilibria in microphase segregation).

Above we have been referring several times to the (a)sphericity of 
the dendrimers. With increasing $f$ and $g$ one would expect that the asphericity, which is a measure for the deviation from a spherical shape, would decrease. We therefore end our analysis by considering this property for linear chains and dendrimers. In Figure 5.7 we present for $f=2,3,4$ and 5 the asphericity $A$ as a function of the generation number $g$, for spacer length $S=20$. Again, for $f=2$ (linear chains) this means that there are $2 g+1 \mathrm{MC}$-beads in the MC-SCF model and the degree of polymerisation is $N=2 g(S+1)+1$. In the cell model the spherical symmetry is imposed and therefore the cell model always has an asphericity of zero. As a result we can only discuss the performance of the MC-SCF hybrid (dashed lines) in relation to the FJC result (dotted lines).

Inspection of Figure 5.7 shows that the MC-SCF hybrid performs very well with respect to fluctuations in shape. For the dendrimers $(f>2)$ in Figure 5.7p,c and d, two generations are enough to let the difference in asphericity with the MC-FJC model, become less than the uncertainty margin. For linear polymers a few more branch points are needed before the asphericities are the same, within the uncertainty margins, as those of the FJC model. Although the confidence interval is still quite large, the MC-SCF model thus seems to capture the asphericity of the dendrimers well. In both models we see that for linear chains the asphericity goes to roughly a value of 0.4 and as expected the values for $A$ tend to be a strongly decreasing function of the number of generations in the dendrimer $g$. The absolute values of $A$ decrease with increasing $f$.

\section{Conclusion and Outlook}

With the SF-SCF theory properties of complex polymeric systems can be obtained in seconds of CPU time. The SF-SCF theory however does not treat the correlations between the polymer segments correctly. This may in turn have consequences for interactions which strongly depend on such correlations, such as excluded volume interactions. To asses how big the errors due to neglecting part of the correlations are, we used dendrimers as a model system, because excluded volume interactions played an important role in them. In an attempt to bring back 
some of the correlations in the SF-SCF theory, we created the MC-SCF model. In this model the positions of some of the segments are specified while the remaining segments are treated with the SF-SCF theory. Subsequently, the segments were moved with a Monte Carlo algorithm. As a reference system we used a freely jointed chain model (MC-FJC) with hard beads. Both the cell model and the MC-SCF model show quantitative differences with the MC-FJC model. More importantly though the cell model also shows qualitative differences such as a different scaling for the radius of gyration of the dendrimers. We also found that the cell model predicts a bimodal distribution for the first branch point from the center for large $f$ and $g$. This suggests that there are two populations of conformations inside the dendrimer. These were, however, not found by the other two fundamentally more correct approaches. We attribute the differences between the results of the MC-SCF and the MC-FJC model and those of the cell model, to an overestimation of the long range- and underestimation the short-range excluded volume interactions in the cell model. Together with the property that in SCF the bimodality can erroneously be created by 'fractional' branchpoint redistributions, this can explain why a two sub-population status is found for the cell model when both $f$ and $g$ are large. This flaw of $\mathrm{SCF}$ in the high crowding limit came as a surprise, because in the high crowding limit (large $f$ and $g$ values) one usually finds that the flaws of the mean field approximation are relatively small. In general one should thus be careful when interpreting results from the cell model. The MC-SCF model is fundamentally better at describing dendrimers than the cell model, as it gives the correct qualitative behaviour. It is however computationally far more costly than the cell model. In an attempt to keep the CPU time within bounds we introduce the concept of 'sub boxes'. These sub boxes are significantly smaller than the overall volume and this allows to compute the segment densities of fragments of the molecule on a 'local' level. Even with this implementation trick, the MC-SCF method was computationally expensive. In the current implementation the MC-FJC model was still faster than the MC-SCF model. The type of Monte Carlo moves we used however differed between the models and a fair comparison is therefore not possible. We expect that the difference in CPU time between MC-SCF and the MCFJC method will be less for denser systems, thus for short spacers and 
sufficiently large values of the number of generations $g$, and number of spacers per branch, $f$. Together with possible extra optimisation steps in computing the volume fractions per spacer (better implementation of sub boxes) and smarter Monte Carlo moves we may find a MC-SCF hybrid that can outperform simulations that take all excluded volume interactions explicitly into account. 
Chapter 5 


\section{CHAPTER 6}

\section{General Discussion}

Now that I have reached the end of my $\mathrm{PhD}$ it is time to look back at what we have learned, what could have been done better, which questions remain and how these can be answered in the future.

\section{Interactions between Telechelic Polymer Micelles}

In chapter 2 we determined how micelles with telechelic polymers interact as a function of the number of telechelic polymers $f$ per micelle, the solvent quality $\chi$, and the length of the polymers $N$. At short inter micelle distances there is repulsion due to the overlap of the coronas. At longer inter micelle distances there is a weak attraction, because the number of possible polymer conformations and therefore the entropy increases if the polymers can also form bridges.

We found that the strength of the interactions between the micelles depends on their surroundings. The depth of the attractive well decreased by almost a factor 2 and the range over which the interactions are repulsive also increased when going from two isolated micelles to two micelles in a FCC packing. The reason for this behaviour is that when two isolated micelles are pushed into each other, the coronas can move out of the way while if they are already surrounded by others this is not possible. This means that the interaction potential is not pairwise additive. We could thus not simulate the micelles as a single particle with pair wise potentials. Therefore we had to look for a different way to simulate a gel made of these micelles.

Determining the correct non pair wise potentials to simulate the system will be a difficult and time consuming enterprise, although with the reward of being able to describe a large piece of telechelic polymer gel. 
For the self consistent field theory we do not have to specify the interaction potentials between the micelles, they emerge from the interaction between the monomers. SCF, however, typically finds the polymer distribution with the lowest free energy, this would most likely result in the micelles being ordered in a crystalline way. A real system however fluctuates around the state with minimal free energy. A plain SCF model would not incorporate these fluctuations and would thus not give a good picture of such a gel. We therefore decided to introduce these fluctuations into the SCF model by constraining the end groups of the telechelic polymers in nodes, and move these nodes with a Monte Carlo method. We show some preliminary results of this hybrid model in chapter 3 and give a more extensive discription in chapter 4.

\section{MC-SCF Hybrid for Networks of Telechelic Micelles}

In chapter 3 we show the results of a simulation of a gel made of telechelic polymers with a weakly charged middle block adsorbed onto a wall. We originally wrote it for a festschrift in honour of our colleague Herman van Leeuwen. ${ }^{113}$ Herman van Leeuwen is an electrochemist who worked, amongst other things, on methods to measure the concentration of metal ions in (natural) water. Two techniques that are used for this are DGT(diffusive gradient in thin films) and DET(Diffusion Equilibrium in Thin films). In DET a thin layer of gel, which has chemical groups that can reversibly bind with metal ions is placed in the water. After a certain time an equilibrium is established between the water and the gel and the gel is enriched in metal ions. From the metal concentration in the gel one can then derive the concentration in the water. In DGT a metal ion binding resin is covered by a thin layer of gel. This layer of gel limits the rate at which the metal ions can reach the resin and the quantity of metal ions in the resin thus corresponds to a time averaged concentration.

For the impact of these metal ions on organisms and the environment it is not so important what the quantity of these metal ions is but how bioavailable they are. The concentration of free metal ions in the water is a good measure for this.

It was assumed that the maximum size of the pores in these gels was very small $<10 \mathrm{~nm}$ and that thus no particles could penetrate 
the gel. Around the time I started my $\mathrm{PhD}$ it was however discovered that much larger particles, $10 \mathrm{~nm}<r<130 \mathrm{~nm}$, could penetrate these gels. $\frac{114 \mid 115]}{1}$ Natural water often contains organic particles made of fulvic and humic acids that can bind metal ions. If they diffuse into films used in DET, a much higher metal ion concentration may be measured than the concentration of the free ions in solution.

From this perspective it is understandable that the structure of gels is important for the topics Herman van Leeuwen studied. We therefore simulated a small piece of a physical gel, made of telechelic polymers with negative charges, bound to a surface. Usually, chemically bound rather than physically bound gels are used in DET and DGT applications. Each individual snap shot of a physical gel should however be an example of a structure that a chemically crosslinked gel can take and our physical gel should therefore be representative of chemically crosslinked gels.

As the time before the submission deadline was rather short, we could only simulate a relatively small piece of gel for a limited amount of time. In this small simulation we however saw that there were large fluctuations in the gel in agreement with the observation that large particles could penetrate in these gels.

In hind sight it is surprising that the gel stayed intact during the simulation. First of all, we used a Flory-Huggins parameter of $\chi=0$ which means there is no attraction between the polymer segments to compensate for the steric hindrance between them. Second, the system we simulated was very small in the $\mathrm{x}$ and $\mathrm{y}$ direction. As hydrogels have low surface tensions this should make it relatively easy for the gel to break into two pieces. Also based on the interaction potentials we found in chapter 2 we did not predict phase separation for this system. And finally, we did not observe phase separation for our system in chapter 4 with on average $f=2.5$ polymers per node and $\chi=0$ or even for $f=2.5$ and $\chi=0.5$. Although this may have had other reasons; the polymer concentration may not have been low enough as the lowest concentration we modelled was $3.1 \%$ and the number of Monte Carlo steps may also have been to short for nucleation of the phases to occur.

In theory the charges on the polymers can help to stabilize the gel phase a bit. The electrostatic repulsion between the segments of a polymer increases the persistence length of the polymers. As the 
polymers become stiffer they prefer to form bridges instead of loops as they have to bend less to form a bridge. $\frac{116}{1}$ This can increase the bridging attraction between the micelles. The SCF model however does not really captures this effect, because in the SCF model the polymer density is smeared out around the node. A polymer will thus feel its own electrostatic repulsion much less.

Most likely the system has not been sufficiently equilibrated. The nodes have to distribute themselves over the volume by diffusion. This diffusion is however counteracted by the weak attractive forces between the nodes. The nodes will thus spread even slower than ideal particles.

Another imperfection in this chapter is the way in which the electrostatic interactions are treated. These interactions were calculated using the Poisson equation. For our mean field model this however means that we implicitly also calculate the interaction of charged particles with themselves. This prevents the SCF model from simulating systems like complex coacervates, which consists of oppositely charged polymers that can phase separate due to their mutual attraction. Because we use a 3D lattice, each lattice site contains only one segment. It is therefore possible to subtract the self interaction of the segments. This correction probably is sufficient to cause attraction between oppositely charged polymers, it however does not give an accurate description of this attraction. A more thorough description would have to take correlations between the position of the segments into account. One way to do this would be to pinpoint the charges to specific lattice sites and move them with a Monte Carlo algorithm, although this may not be feasible for systems with high charge densities.

In chapter 4 we model homogeneous gels made of telechelic polymers, with a number of polymers ranging from $f=2.5 \cdots 10$ and a Flory-Huggins solvent quality parameter varying from $\chi=0 \cdots 0.5$, over a range of volume fractions $\varphi=0.03 \cdots 0.5$. We determined the distribution of the telechelic polymers with SF-SCF theory, while the ends are constrained in nodes which are moved with a Monte Carlo method. With this hybrid method, we are able to determine the radial distribution functions for these gels. To determine the structure factor and the osmotic compressibilities of these gels we developed a new method to correct the radial distribution function for the small size of our simulation volume. 
For some of the combinations of $f$ and $\chi$ we observed phase separation. Our simulation volumes were however to small to determine the phase diagram accurately. Because of the small volume there will be significant Laplace pressures in the system from the strongly curved interfaces. This suppresses phase separation and a macroscopic system may thus phase separate for lower values of $f$ and $\chi$. Furthermore it can take a significant amount of computation time before nucleation of the phases takes place. Instead, it would be better to implement a Gibbs ensemble ${ }^{97}$ to study the phase behaviour of micelles with telechelic polymers. Our findings agree with some of the experimental literature, where phase separation is observed for roughly the same values of $f$ and $\chi \cdot \frac{45 \mid 117}{}$ After correcting for the difference in polymer length using the Daoud Cotton model ${ }^{58}$, the shape of the structure factors is also similar to those found by Bagger-Jörgensen et al.117

\section{Dendrimer Models}

In chapter 5 we compared how well three different methods describe dendrimers, the classical SF-SCF cell model, the new Monte Carlo SCF hybrid and a freely jointed chain model which functions as the reference model. Because we have chemical bonds rather than physical ones, each chain has to start and end at a specific branch point. Therefore a separate propagator is needed for each chain. Because the propagators for each chain will be zero in most of the simulation volume, we introduced sub boxes so we only need to calculate them in small sub volumes.

These sub boxes can also save computation time when studying the phase behaviour of the gels from chapter 4 . If the phase, with which the gel is in equilibrium, has a low density of micelles, most of the volume will be free of polymers. Computation time can then be saved by only calculating the propagator in the volume around the micelles.

We found that both the cell model and the hybrid underestimated the radius of gyration $\left(R_{g}\right)$ of small dendrimers because they locally underestimate the excluded volume interactions. The hybrid model shows roughly the same scaling for $R_{g}$ as the freely jointed chain model, when the number of generations of the dendrimers is increased. The cell model, however, shows a more rapid increase of $R_{g}$ and even over takes the freely jointed chain model. This happens because the cell model over 
estimates the steric repulsion between segments that are distant from each other in the molecule. The excluded volume of chain fragments that are connected to each other will overlap. In the cell model these chain fragments are however smeared out over a large volume and there is thus little overlap between their excluded volumes. In the hybrid model some of the segments are located in a specific lattice site. This reintroduces the correlations of the positions on the larger length scales and therefore also a large part of the overlap of the excluded volumes.

The cell model also wrongly predicts a bimodal distribution of the first branch points for dendrimers with a high number of generations and a large number of branches per branch point. This may be because the cell model allows a fractional amount of a branch to stay in the centre and not swell. For the other models only an entire branch can not swell and stay in the centre. There is however not enough room in the centre for an entire branch. Therefore the hybrid model and the freely jointed chain model do not show a bimodal branch point distribution.

In hind sight it may have been better to use a hexagonal grid instead of a simple cubic grid for the hybrid model. For the largest dendrimers the acceptance rate of the moves of the first branch points was very low. The spacers near the centre are already quite stretched and moving the branch point by two lattice sites causes quite a large change in the free energy. With a hexagonal grid the branch points only needs to move one lattice site. A disadvantage of using a hexagonal grid is however that each lattice site has twice as many neighbours and calculating the propagator therefore takes longer.

It is also striking that the calculations for the more detailed reference system were faster than those of the hybrid model. The advantage of the self-consistent field method is that it averages over all configurations of the system and thus gives an ensemble average in a single step. In the hybrid method, most of the degrees of freedom are still treated with the self-consistent field approximation. One thus still averages over a substantial part of the configurational space with each Monte Carlo step. One would therefore expect that it would still have a speed advantage over the more detailed freely jointed chain model. For the hybrid method we however used a rather simple translational Monte Carlo move, while the freely jointed chain model used a pivot move which results in bigger displacements of the segments. Using such pivot 
moves in our hybrid simulation may thus also improve the efficiency of that method.

One way to do this would be to keep track of the positions of the branch points with a much higher resolution than that of the lattice. This is required because rounding off the coordinates will result in a different distance relative to the segment to which the branch point was pivoted. Due to this change in distance the reverse move may no longer be possible, i.e. the distance would be too small or too big to reach the lattice site the Monte Carlo move started from. This would mean that detailed balance would be violated and that the move probably will not sample the states according to their Boltzmann weights. Besides the Pivot move translational moves should be done to change the distances between the branch points. For calculating the SCF free energy the branch point positions are rounded to the nearest lattice site.

The usefulness of these pivot moves is however also limited. When I tried to simulate even larger dendrimers with the freely jointed chain model, the acceptance rate of the pivot moves rapidly dropped. As the number of generations increases the concentration within the dendrimer also increases and the number of beads moved per pivot move increases as well. The chance that no overlap occurs after a pivot move therefore rapidly decreases. A move in which a smaller part of the dendrimer is moved may be better in this case.

For $f=3$ branches per branch point, the branch points can be displaced with a mirror move. A mirror move can be done by randomly selecting three beads in the arms connected to the branch point and subsequently mirroring the positions of all segments closer to the branch point along the chain in the plane going through these beads.

For $f>3$ this is not possible, as one of the bound chains would be broken. Instead a multiply concerted rotation move could be used to displace the branch points. $\frac{1181119}{1 n}$ such a move the branch point is displaced by changing the dihedral angle between the branch point and three of the segments in one of the branches connected to the branch point. Subsequently, three segments in the other chains connected to the branch point are moved by calculating all possible sets of dihedral angles that would connect these three segments to the rest of the molecule while maintaining the same bond distances and bond angles. There is a limited number of ways to do this, although it is also possible there 
is no solution in which case the move is rejected. One of these sets of dihedral angles is chosen as the new state.

To be sure that this type of move is a valid Monte Carlo move, the acceptance rule has to be defined such that detailed balance is maintained. Therefore the acceptance rule has to be expanded because the probability with which a move from A to B is attempted when the system is in state A can be different from the probability with which a move from $\mathrm{B}$ to $\mathrm{A}$ is attempted when in state $\mathrm{B}$. A further correction is needed because in the process of generating the new configuration a switch between two different coordinate systems takes place.119

With these multiply concerted rotation moves and wheel moves for the stretches without branch points, it should be possible to simulate freely jointed chain dendrimers of hard spheres with a higher number of generations/density. It will be interesting to see how well the different simulation and analytical methods predict the size of the dendrimers as the number of generations and thus the amount of crowding increases.

\section{The Future}

The simulations of the telechelic polymer gels and dendrimers took a long time, sometimes even months on a single CPU core. Using a GPU helped to speed up these calculations but also placed constraints on the size of the system as the amount of memory available on the GPU was limited. This was one of the reasons why even larger dendrimers were not simulated with the hybrid model. During my $\mathrm{PhD}$ the computational throughput however has increased by a factor 6 to 14 and it is expected that it will keep increasing in the near future.

The efficiency of our simulation could be further improved by making smarter Monte Carlo moves, for example by moving the particles in clusters. Larger systems could then be modelled and artefacts due to the limited number of nodes will be reduced. The hybrid method thus seems a useful tool to determine the static properties of these gels at high polymer volume fractions. For systems with low polymer volume fractions the SCF model may be less suitable because the computation time scales with the volume rather than the number of particles although this can partially be alleviated by using sub boxes. 


\section{Models for studying Dynamics of telechelic polymer networks.}

The model, as presented in chapter 4, is not suitable to determine the dynamic properties of telechelic polymer gels. First of all the hybrid model allows for half a bridge to exist, while in a real system there can only be an integer number of bridges between two nodes. In chapter 5 we have already seen a way to solve this, although we would still need a method for breaking and reforming the bridges and loops in the system.

Another challenge is determining a way to move the nodes. It is in principle possible to use Monte Carlo moves to determine the time evolution of a system, for example by using the kinetic Monte Carlo method. This however requires that the transition probabilities between different states are known. For our hybrid method we do not know exactly what these transition probabilities are. We could determine them by calculating how the free energy would change if a node is moved in each direction. This would however take a lot of computation time.

An alternative would be to move the nodes with a molecular dynamics algorithm. For this we need to determine the forces on the nodes. With the given segment potentials we can determine the change in free energy if we would move the node a little bit into a neighbouring lattice site. Given the coarseness of our lattice, this force can probably not be extrapolated to the determine the free energy in the neighbouring lattice sites sufficiently accurate to determine the transition rates for the dynamic Monte Carlo moves. Smaller lattice sites could be used, but this will drastically increase the computation time.

We could however use these forces if our moves are over considerably shorter distances than the size of our lattice sites. In that case only a small part of a node would move into the neighbouring lattice site. The lattice sites would then have to be split in two, one part containing the node and one part containing the solvent and the corona. Spreading the node over more lattice sites will increase the entropy and the nodes will therefore preferably be on the corners of the lattice sites. An additional potential field will therefore be needed to spread the node positions homogeneously over the lattice sites.

Because the nodes are moved only a small distance, the density dis- 
tribution will not change much. An interesting option would therefore be to calculate the polymer distribution using a mesoscale dynamics method. ${ }^{1201121}$ With this method the polymer distribution is updated using Ficks diffusion laws and a random contribution due to thermal noise. This method will require fewer iteration steps and will therefore compensate for the smaller step sizes of the nodes. This has the additional advantage that we no longer have to assume that the time scale on which the polymers relax is much shorter than the time scale on which the node positions relax.

All these methods however still have the disadvantage that entanglements are not taken into account. This may not be vital for a homogeneous network but in a mixture of two networks these entanglements carry the forces between the networks. Without entanglements the dynamics of such double networks can thus not be modelled. At present there is no method to capture these entanglements in the SCF model. To model double networks a different approach is thus needed. It is therefore interesting to look at what has already been done in literature to simulate networks of these telechelic polymers.

P.G. Khalatur and A.R. Khokhlov wrote several articles on the simulation of solutions of telechelic polymers. In one of these studies they used a bond fluctuation model to study the assembly of the telechelic polymers in $2 \mathrm{D}$ and $3 \mathrm{D} \cdot{ }^{122}$ Later they made an MD model in which the telechelic polymers are modelled as a short chain of 8 or 16 particles which interact via a repulsive Lennard-Jones potential. ${ }^{87}$ The bond lengths were kept constant and the end groups attracted each other at short ranges. They induced shear by applying a force, to the particles, with a size that depends as a sinus on the height of the simulation box. With this method to apply shear there are however two regions in which the shear is in opposite direction. The simulation volume was however very small so each of the sheared layers was only about two micelles thick. This is far to thin to observe bulk behaviour. It would have been better if they had applied Lees-Edwards boundary conditions, although the system would still be to small to describe the rheological behaviour.

They also used this model to study the effect of the stiffness of the polymers and found that increasing the stiffness of the polymers gave bigger micelles which had more bridges. ${ }^{116}$ Their model can be improved further by using softer potentials between the beads which are 
more realistic for interacting polymer coils and using a more spring like potential between the beads. By using the Twentanglement algorithm chain crossings could be prevented. 123

Their system was roughly half the size of the box used for the gel simulation in chapter 4. They do have more noise on their data, but considering that they did their simulations more than a decade before the start of my Phd, their model can not be much slower than the hybrid method we used for our gels. It should thus be possible to scale up their model to a similar size as our gel simulation. This may be big enough to simulate the behaviour of the gel under shear when the system remains homogeneous. If phenomena like shear banding and fracture occur the simulation volume will, however, not be big enough.

Due to the relatively detailed nature of this model the time steps should be relatively small. The binding energy of the anchors therefore needs to be low, so the system can relax within the simulation time. This leads to less well defined micelles as the system is close to the critical point. The shear rates required to observe interesting behaviour in the simulation will therefore also be much higher than experimentally accessible. Rheological behaviour like strain stiffening also depends on the strength of these anchors. To some extent the results from these simulations can be extrapolated to experimentally accessible systems. It would however be nice if we could simulate systems with more micelles on longer time scales. This can be achieved by further coarse-graining.

Cass et al. ${ }^{88}$ did this by modelling a telechelic polymer as two particles connected by a harmonic spring. These particles repel each other with a repulsive Gaussian interaction. These particles are attracted by nodes which are placed in the centre of mass of the particles they are attracted to. The particles were moved with Brownian dynamics. They compared their data with experiments of end-capped PEO. Compared to experiments their simulations underestimated the increase in zero shear viscosity with increasing polymer concentration. It thus seems that their model did not capture all essential aspects of the telechelic polymers. Their system was also relatively small with 500 polymers, only twice the number Khalatur used.

Sprakel et al. $\frac{49}{4}$ were more ambitious and coarse-grained entire micelles using the RAPID method.124 This allowed them to model 6750 micelles, enough to observe phenomena like shear banding and fracture. 
They used transient potentials, which means that the strength of the interactions between two micelles depends on their history. The longer two micelles have been next to each other the more likely it is that a bridge has been formed. They therefore kept track of the amount of bridges $n_{i j}$ that would on average be formed between two micelles with these trajectories.

The micelles repelled each other via a harmonic potential with a sufficiently large prefactor that the particles cannot overlap and weakly attracted each other up to slightly longer ranges. There was an additional contribution of a harmonic potential based on the difference between the equilibrium amount of bridges $n_{e}\left(r_{i} j\right)$ at inter particle distance $r_{i j}$ and $n_{i j}$ the amount of bridges between the particles based on their history. This potential effectively reduces the rate at which the distance between the particles can change. The idea behind this is that when the two micelles approach each other quickly the corona's have not had time yet to inter penetrate and the repulsion is thus stronger, while if the particles are pulled apart the stretched bridges pull the particles back together.

With this model they observed shear banding and fracture for concentrated solutions of telechelic micelles. They also determined properties like zero shear viscosity and fracture stress.

Coarse-graining to the level of single micelles thus seems a promising approach. The model of Sprakel et al. however still has some flawed assumptions. In a real gel there is either a bridge or no bridge instead of a fraction of a bridge. This will result in a less homogeneous distribution of the forces between the micelles and a stronger attraction between micelles connected by a bridge. The force they used for the stretching of the bridges is also not correct. At large extensions the potential levels off and the attractive force between the particles goes to zero. The equilibrium number of bridges at this distance is indeed practically zero. There is however an energy barrier for detachment of the chains. In a sheared sample there will therefore still be chains that are strongly stretched and these chains will carry considerable forces.

As Sprakel et al. already mentioned ${ }^{49}$ and I showed in chapter 2 the interaction potentials are not pair wise additive, but also depend on the surroundings of the micelles. At a locally low concentration the net attraction will be stronger than at a locally high concentration. 
This effect was not incorporated in their model. This may explain why Sprakel et al. $\stackrel{49}{ }$ did not observe phase separation for these gels, while we did see this in chapter 4 .

Due to these shortcomings their model will not describe telechelic polymer gels very accurately. Its speed however makes it alluring to try to find potentials that describe the system better while still coarsegraining at the whole micelle level. There are several modifications which could make the model more accurate:

- One could use a crowding parameter based on the number of surrounding micelles and the distance to these micelles. Based on this crowding parameter the strength of the repulsion between the micelles could then be adjusted.

- Another improvement would be to keep track of where the polymers are. For each micelle one could keep track of the number of loops, to which other micelles it is connected, and with how many bridges they are connected.

- Based on the forces on the polymers the dissociation rate of the end groups can be adjusted. Once an end group has dissociated it will start to sample the volume surrounding its other end until it encounters another node. If the time scale at which this happens is longer than the typical time step we will also need to keep track of how many dangling end each node has. Other wise we can immediately assign a new node to the end group.

- Instead of Brownian Dynamics we could also use Dissipative particle dynamics which gives more realistic hydrodynamic behaviour and could for example also account for the friction between the coronas.

- A more realistic potential should be used for the force required to extend the polymers. For the freely jointed chain and worm like chain numerical approximations are given by Petrosyan. 125

- Just as in more detailed models we should also account for entanglements. Although the pairs of micelles connected to the entangling bridges need to be pulled apart before an entanglement 
can form, the energy barrier for this will not be high enough to prevent their formation.

To determine the exact values for the parameters needed in the above mentioned modifications we could use the SCF model or a model similar to the one used by Khalatur. $\frac{116}{16}$ With these modifications sufficiently large volumes of telechelic polymers networks can be modelled to study their rheological behaviour, assuming that the relaxation time of the polymer coils is short relative to the time scale at which the nodes move. If this is not the case, such as for example for collagen, a less course-grained model may be needed.

\section{Conclusion}

In chapter 2 we showed that the interactions between the micelles of telechelic polymers are not pairwise additive. We therefore chose to use the Scheutjens Fleer self-consistent field method to model these telechelic polymer micelles, because this method does not require predefined interaction potentials for the micelles. The SF-SCF method however does not take fluctuations into account. Therefore the SF-SCF method was combined with a Monte Carlo method which moved the micelles and in this way introduced fluctuations in the model. We simulated small pieces of telechelic polymer gels, with a varying number of polymers per micelle, solvent quality and concentration. Structural properties like the radial distribution functions and the compressibility were successfully determined. By pinpointing some of the segments of a polymer on specific lattice sites we also introduce more correlations between the different parts of a polymer. This gave an improved description of dendrimers compared to the classical SF-SCF model. The hybrid model we introduced is therefore a suitable method to study the equilibrium properties of dendrimers and telechelic polymers gels. For the description of the dynamics of these systems the SF-SCF model is less suited, as the lattice is rather coarse and the SF-SCF model does not take entanglements into account. 


\section{Summary}

Polymers are very important in today's society. They are used in many everyday objects such as bags, paint, tires, soft contact lenses and diapers. In the latter two applications the polymers form hydrogels, which are water filled polymer networks. The goal of the $\mathrm{PhD}$ project, which I worked on together with two other PhD students, was to get a better insight in how the properties of the polymers affect the properties of these hydrogels. I developed computer simulation methods to study two classes of polymers, telechelic polymers and dendrimers.

A telechlic polymer is a polymer whose end groups bind to each other, for example by hydrophobic interactions or specific hydrogen bonds. If many of these polymers bind together a flower-like micelle is formed, with the end groups forming the heart and the loop-forming middle blocks as the petals of the flower. The steric hindrance between these 'petals' limits the number of polymers in a micelle. If two micelles are adjacent, a polymer can also form a bridge between them. This results in an entropic attraction because the polymers can now form both loops and bridges and thus have more possible conformations. This attraction can become so strong that phase separation occurs.

If the concentration of the polymers is high, there can be enough bridges between the micelles to form a percolating network and in that case a gel is formed. The micellar cores form the nodes in this network. A schematic representation is given in Figure 1.3. If the binding energy of the end groups is low enough that they can sometimes spontaneously detach, such a gel can heal damage to its network structure.

Micelles and polymer are difficult to observe experimentally especially during rheological measurements. In computer simulations there is no observational limitation. The amount of detail is however limited by the available computation time.

To save time, multiple particles are treated as one particle. As more particles are grouped together, bigger systems can be simulated on longer time scales. Ideally, entire micelles are thus simulated as single particles. 
Usually it is assumed that the forces between two of these composite particles are pair wise additive, i.e. the force between them does not depend on the positions of other particles. To test whether this assumption holds for telechelic polymer micelles, I calculated the interaction energy between two micelles, for different 3D particle geometries: an isolated pair, a chain, a simple cubic ordering and a face centred cubic ordering. The number of polymers per micelle, the solvent quality, and the length of the polymers were also varied.

These calculations were done with the Scheutjens Fleer self consistent field (SF-SCF) theory, which is a lattice based model that determines the polymer distribution with the lowest free energy. The interaction energy of the polymer segments is based on their average environment. SF-SCF is a relatively fast technique because it samples all degrees of freedom in one go.

In chapter 2 we show that the micelles repel each other at short distances but weakly attract each other at a bit longer range. The position of the minimum scales roughly as $N^{3 / 5}$ and $f^{1 / 5}$ in accordance with the Daoud Cotton model. At $\chi=0.5$ the depth of the minimum for the FCC packing is only $60 \%$ of that of the isolated micelles. This makes a big difference for the behaviour of such gels. For example, if the pair potential for the FCC structure is used to estimate the minimal number of polymers per micelle required to cause phase separation, the required number is more than twice the experimentally observed minimum. With the pair potential of the isolated pair even one polymer per micelle would however be enough.

The interactions are thus not pair wise additive and we can therefore not use a method based on pair wise potentials. The SF-SCF method does not make assumptions about the pair potentials, instead the micelle-micelle interaction potential emerges from the interactions between the individual polymer segments. The SF-SCF method finds the state in which the polymers have the minimal free energy. It however does not take the free energy of the nodes into account. We therefore introduced a Monte Carlo algorithm (MC) to move the nodes. This introduces fluctuations in the positions of the nodes and thus gives a more realistic structure.

In chapter 3 we describe a preliminary study of a charged gel bound to a wall. This gel represents the gel layer found in DGT and DET 
devices, which are used to measure metal ion concentrations in water. It was recently discovered that particles can penetrate these gels and it is therefore interesting to study the structure of these gels. We found big structural fluctuations in the gel. The simulation was however too small and too short to draw final conclusions.

Chapter 4 describes a more extensive study of gels using the new SF-SCF MC hybrid. We determine the radial distribution functions as a function of the number of polymers per micelle $f$, the solvent quality $\chi$ and the polymer concentration $\varphi$. From these radial distribution functions we in turn calculate the structure factors and the osmotic compressibilities.

It was striking that, for $f=5$, the height of the first peak in the radial distribution function decreased as the concentration increased. As described in chapter 2, this is initially caused by a decrease in attraction between the micelles as the number of neighbouring micelles is increased. As the concentration increases beyond $\varphi \approx 0.125$, the entire volume is filled. The repulsion now becomes weaker because the difference between the osmotic pressure at the overlapping coronas and the average osmotic pressure becomes smaller. The height of the first peak therefore does not increase. For $f=10$ the height of the first peak did not decrease at high concentrations.

In agreement with some of the literature, we found phase separation for $f=5$ and $\chi=0.5$. Phase separation probably also occurs for $f=10$, but this was not as clear in our simulations. To study the phase behaviour of these gels thoroughly they should be modelled using the Gibbs ensemble. The shape of the structure factors we calculated matched with those in the literature for the same $f$.

We also compared the radial distribution function for $f=5$ and $\chi=$ 0.5 with a radial distribution function of particles with pair wise additive forces with potentials based on those in chapter 2. For $\varphi=0.125$ this gave a good match but not for lower and higher concentrations.

With this new hybrid method we not only introduce fluctuations, but we also pinpoint the positions of some of the segments and thus introduce positional correlations in the SF-SCF model. We therefore wondered whether pinpointing the segments would improve the treatment of excluded volume interactions within the SF-SCF theory.

In chapter 5 we test this by comparing how well the new hybrid 
method describes dendrimers compared with the classical SF-SCF theory and a more detailed freely jointed chain (FJC) model with excluded volume. In the classical SF-SCF theory we used the cell model, which assumes that the polymer density profile is spherically symmetric. With the hybrid method we specify the position of the branch points and move these with a Monte Carlo algorithm. Because each spacer starts and ends at different branch points a separate propagator is needed for each spacer. To save computation time we therefore introduced sub volumes, so we only needed to calculate the propagator for the spacers in those parts of the volume where the probability to find the spacer is not negligible. We simulated dendrimers with 3,4 or 5 polymers per branch point up to 7,4 and 4 generations respectively. The middle blocks had a length of 20 or 50 segments.

Both the cell model and the hybrid method underestimate the radius of gyration of the dendrimer. In contrast to the cell model, for which the radius of gyration increased too fast with an increasing number of generations, the hybrid did give the same scaling as the more detailed FJC model. This flaw in the cell model is attributed to an overestimation of the excluded volume interactions between different branches. The cell model also wrongly predicts the distribution of the first branch points for dendrimers with strong crowding. In the hybrid and the FJC models the spacers connected to the central segment of the dendrimer are strongly stretched. With the cell model a fraction of the spacers is however unstretched. This is probably because for the cell model a fraction of an arm, for example 0.4 arms, can stay in the centre, while the other models only allow an integer number of arms. There is however not enough room in the centre for an entire arm and therefore all the first generation spacers are stretched for the FJC and the cell model. Although much slower than the classical SF-SCF method the hybrid method thus gives a better description of the dendrimers and the excluded volume interactions.

This newly developed hybrid method extends the range of problems which can be studied with SF-SCF theory. The model is however not suitable for studying dynamic properties of such systems because of the coarseness of the lattice and the lack of a way to prevent polymers chains from crossing. To study dynamic properties a Brownian dynamics or dissipative particle dynamics simulation, in which the polymers 
segments or even entire micelles have been coarse-grained to single particles will be needed. The development of the correct interaction potentials between these particles is however challenging, especially if whole micelles are coarse-grained. 


\section{Samenvatting}

Polymeren zijn moleculen die bestaan uit een keten van aan elkaar gebonden kleinere moleculen. Elk van die kleine moleculen wordt een monomeer genoemd. Polymeren lijken in dat opzicht wel op een ketting van klik-kralen. Een aantal voorbeelden van polymeren wordt gegeven in Figuur 1.1. Polymeren zijn erg belangrijk in de huidige maatschappij. Ze worden in allerlei alledaagse toepassingen gebruikt, zoals plastic tasjes, verf, banden, zachte contactlenzen en luiers. In de laatste twee toepassingen vormen de polymeren hydrogelen. Dit zijn met water gevulde polymeernetwerken. Het doel van het project was om een beter inzicht te krijgen in hoe de eigenschappen van deze polymeren, zoals hun lengte en hydrophobiciteit, de macroscopische eigenschappen, zoals hoeveel kracht het kost om de gel te vervormen of de gel te breken, beïnvloeden. Ik werkte hierbij samen met twee andere PhD studenten. Zij deden experimenteel werk terwijl ik computersimulaties ontwikkelde. Ik heb hierbij twee groepen polymeren bestudeerd, dendrimeren en associërende polymeren.

Een associërende polymeer is een polymeer waarvan de uiteindes op een of andere manier ergens aan kunnen binden. In deze thesis gaat het specifiek over uiteindes die bij elkaar binden. Deze binding kan bijvoorbeeld komen doordat allebei de uiteindes hydrofoob zijn, dat wil zeggen dat ze slecht oplosbaar zijn in het oplosmiddel, of doordat ze specifiek aan het oppervlak van een heel klein deeltje binden. Als beide uiteindes op dezelfde plek binden wordt een lus gevormd. Het aantal polymeren dat zo aan elkaar kan binden is beperkt. Op een gegeven moment gaan de lussen, die door de middenblokken gevormd worden, elkaar in de weg zitten. Zo'n groepje van een beperkt aantal moleculen wordt ook wel een micel genoemd. Als twee van zulke micellen naast elkaar liggen kunnen er ook bruggen gevormd worden, waarbij het ene uiteinde van de polymeer in de ene micel zit en het andere uiteinde in de andere micel.

Aangezien er nu ook bruggen gevormd kunnen worden neemt het aantal manieren waarop de polymeren over de micellen verdeeld kunnen 
worden toe. Omdat elke combinatie, van de posities van de micellen en de verdeling van de polymeren over die micellen, even veel kans heeft om voor te komen, zullen de micellen vaker naast elkaar liggen. Effectief trekken de micellen elkaar dus aan, en deze attractie wordt entropische attractie genoemd. Deze attractie kan zo sterk zijn dat fasescheiding optreed en het ene deel van de oplossing dus een hogere concentratie micellen heeft dan het andere deel.

Als de polymeerconcentratie hoog genoeg is kunnen er zoveel bruggen worden gevormd dat er een aaneengesloten netwerk ontstaat. De kernen van de micellen, waar alle polymeer uiteindes bij elkaar komen, vormen de knooppunten in dit netwerk. In Figuur 1.3 is zo'n netwerk schematisch weergegeven.

Als de gel beschadigd wordt zullen de bruggen tussen de knooppunten verbroken worden. Wanneer de bindingsenergie van de uiteindes echter niet al te hoog is zullen ze af en toe ook spontaan loskomen en kunnen er weer nieuwe bruggen gevormd worden. In dat geval kan de gelstructuur zich dus herstellen. Op tijdschalen korter dan de tijd die gemiddeld verstrijkt tussen het binden en weer loslaten van de uiteindes zal de gel zich als een vaste stof gedragen. Op langere tijdschalen zal de gel zich meer als een vloeistof gedragen.

Het is moeilijk om met experimenten te zien wat er in polymeergelen gebeurt. De polymeren zijn veel te klein om onder een microscoop te zien. Computersimulaties hebben deze beperking niet, maar de grootte van de gel die gesimuleerd kan worden is wel beperkt door de rekenkracht die een computer heeft.

Om rekentijd te besparen kunnen meerdere deeltjes samen als één deeltje gesimuleerd worden. Naarmate er meer deeltjes samengevoegd worden, kunnen er grotere systemen over langere tijdschalen gesimuleerd worden. Idealiter zou een hele micel dus gesimuleerd worden als een enkel deeltje.

Meestal wordt aangenomen dat de interacties tussen deze deeltjes paarsgewijs zijn. Dit betekent dat de grootte van de kracht die twee deeltjes op elkaar uitoefenen niet afhangt van de posities van de andere deeltjes. Om te testen of deze aanname klopt voor deze micellen van associërende polymeren, heb ik, in Hoofdstuk 2, de interactie-energie tussen twee van deze micellen berekend, terwijl ze op verschillende manieren omringd waren door andere micellen. Hierbij heb ik ook het 
aantal polymeren per micel, de lengte van de polymeren en de sterkte van de interactie tussen de middenblokken gevarieerd.

Deze berekeningen heb ik gedaan met de Scheutjens-Fleer zelfconsistent veld methode(SF-ZCV). Hierbij wordt de ruimte opgedeeld in vakjes. Elk van de monomeren past precies in zo'n vakje. Monomeren die in de polymeren aan elkaar vastzitten moeten naast elkaar in de vakjes zitten. Er zijn heel veel manieren om een polymeer in deze vakjes te stoppen. Een voorbeeld wordt gegeven in Figuur $2.1 \mathrm{~b}$. Zo'n manier om een polymeer in de vakjes te stoppen wordt een conformatie genoemd. Als er meerdere polymeren gesimuleerd worden kunnen de polymeren elk vele conformaties aannemen. Zo'n set van conformaties noemen we voor nu een configuratie.

Er zijn echter veel te veel configuraties om ze elk afzonderlijk te simuleren. Met een Monte Carlo simulatie probeer je daarom op een slimme manier een aantal onafhankelijke configuraties te genereren om vervolgens, door over deze configuraties te middelen, een schatting te maken van de eigenschappen.

Met de Scheutjens-Fleer zelfconsistent veld methode pakken we het echter anders aan. In plaats van alle configuraties te genereren, genereren we alleen de polymeerconformaties. De polymeren verdelen zich over deze conformaties volgens de Boltzmannverdelingsfunctie, d.w.z. dat de kans dat een polymeer in die conformatie zit omgekeerd evenredig is met de exponent van de interactie-energie. Voor elk van deze conformaties berekenen we de interactie-energie op basis van een gemiddelde omringing. In eerste instantie weten we nog niet hoe ze omringd zijn door andere polymeren en moeten we een begin schatting maken. Als we de interactie-energie voor elke polymeerconformatie bepaald hebben kunnen we uitrekenen hoeveel polymeer er gemiddeld in elke conformatie zit. Op basis hiervan kunnen we dan weer de lokale polymeerconcentratie uitreken. Hiermee kunnen we een nieuwe schatting maken van de interactie-energie van de conformaties. Dit proces herhalen we totdat de verdeling van de polymeren over de conformaties niet meer veranderd en we dus een zelfconsistente oplossing gevonden hebben.

Om de interactie-energie tussen de micellen te bepalen moet de afstand tussen de micellen kunnen variëren. Daarvoor is het noodzakelijk dat de micellen "vast gehouden" kunnen worden. We hebben dit gedaan 
door kleine volumes te maken waar binnen de uiteindes van de micel moeten zitten.

Met deze methode vonden we dat de micellen elkaar als ze dicht bij elkaar zitten afstoten, maar op een iets grotere afstand elkaar juist aantrekken. Hoe sterk ze aan elkaar binden hing af van hoe ze door andere micellen omringd werden. Voor een kubisch vlakgecentreerde omringing(FCC) was de bindingsenergie maar $60 \%$ van die voor een geïsoleerd paar micellen. Als we op basis van deze bindingsenergie het fasegedrag proberen te voorspellen vinden we grote verschillen. Als we uitgaan van de bindingsenergie bij de FCC-omringing, zijn er om fasescheiding te veroorzaken twee keer zo veel polymeren per micel nodig dan experimenteel is waargenomen. Als we echter de paar-potentialen van een geïsoleerd paar zouden nemen zou één polymeer per micel al genoeg zijn.

Om goede voorspellingen te kunnen doen over de eigenschappen van oplossingen van dit soort micellen moet er bij het bepalen van de krachten tussen de micellen dus rekening gehouden worden met de omringing. Dit is bij normale paar-potentialen niet mogelijk en daarom moesten we een andere methode bedenken om dit soort micellen te simuleren.

De SF-ZCV methode was al gebruikt om de paar-potentialen uit te rekenen. Het lag daarom voor de hand om te kijken of deze methode ook gebruikt kon worden voor de gelen van associërende polymeren. Met de SF-ZCF methode kan wel bepaald worden hoe de polymeren over de micellen verdeeld zijn, maar niet hoe de micellen over de ruimte verdeeld zijn. Daarom hebben we de Monte Carlo simulatietechniek gecombineerd met de SF-ZCV methode. Hierbij worden de micellen telkens in een willekeurige richting verplaatst. Leidt dit tot een verlaging van de potentiële energie dan wordt deze verplaatsing geaccepteerd. Als de potentiële energie echter toeneemt dan wordt de verplaatsing geaccepteerd met een kans die exponentieel afneemt met de toename van de potentiële energie. Op deze manier kunnen we ook het effect van de fluctuaties van de micel posities meenemen.

In Hoofdstuk 3 hebben we deze methode gebruikt om een geladen gel geadsorbeerd aan een wand te modelleren. Dit soort gelen wordt gebruikt bij het meten van de gemiddelde concentratie opgeloste zware metalen in water. De ionen van de zware metalen kunnen doordringen in 
de gel en worden in de gel of een onderliggende hars laag geabsorbeerd. Het idee is dat de metaalionen wel door het polymeernetwerk kunnen diffunderen maar dat grotere deeltjes dit niet kunnen. Onderzoekers van onze groep hebben echter ontdekt dat ook grotere humusdeeltjes, waar de metaalionen aan kunnen binden, in de gel konden doordringen. Hierdoor kan de concentratie van vrije metaalionen overschat worden.

In de simulaties zagen we grote poriën in de gel. Deeltjes zouden de gel door deze poriën binnen kunnen dringen, wat in overeenstemming is met experimenten. De grootte van deze simulatie was echter beperkt en dit heeft invloed gehad op de uitkomsten van het model. Er moeten dus geen al te harde conclusies aan verbonden worden. Het ging er meer om, om te laten zien dat de methode in principe werkt.

In Hoofdstuk 4 hebben we een uitgebreidere studie gedaan van gelen gemaakt van associërende polymeren. Hierbij hebben we gekeken naar het effect van het aantal polymeren per micel, de polymeerconcentratie en de interactie-energie tussen het midden blok en het oplosmiddel. Met deze simulaties hebben we structurele eigenschappen van deze gelen bepaald, zoals de radiale distributiefunctie, structuurfactoren en de osmotische compressibiliteit. De structuurfactoren uit onze simulatie waren kwalitatief vergelijkbaar met die uit experimenten en het lijkt er dus op dat deze methode een goede manier is om dit soort gelen te simuleren.

Het was opvallend dat voor vijf polymeren per micel de hoogte van de eerste piek in de radiale distributiefunctie afnam naarmate de concentratie toenam. Voor lage concentraties komt dit doordat de attractie tussen een paar micellen afneemt naarmate ze door meer andere micellen omringd worden. Bij hoge concentraties zijn er geen "gaten" meer in de gel waar de lokale polymeerconcentratie praktisch nul is. De lokale verschillen in de polymeerconcentratie zijn hierdoor kleiner. Hierdoor maakt het voor de sterische hinder, tussen de lussen die uit de micellen steken, minder uit waar de kern van de micel zich precies bevind. Dit leidt er toe dat de oplossing minder geordend is en daardoor neemt de hoogte van piek in de radiale distributie af. Dit is zichtbaar in Figuur 4.6 .

Bij een interactie-energie van $0.5 \mathrm{kT}$ tussen de monomeren en het oplosmiddel, en vijf polymeren per micel, vonden we dat er fasescheiding optreedt. Dit is in overeenstemming met de literatuur waarin andere 
onderzoekers ook een grens vonden van ongeveer vijf polymeren per knooppunt voordat fasescheiding optrad. De opzet van de simulaties was vooral gericht op het bepalen van de structuur van de homogene gelen en we hebben daarom geen fasediagram kunnen maken. We hebben de uitkomsten van deze simulaties ook vergeleken met een simulatie waarbij de hele micel als een enkel deeltje gesimuleerd werd. Hierbij hebben we paar-potentialen gebruikt die gebaseerd waren op de potentialen die we in Hoofdstuk 2 gevonden hebben. Voor een volumefractie van $12,5 \%$ kwam de radiale distributiefunctie redelijk overeen, maar voor hogere en lagere volumefracties waren er grote verschillen. Dit laat zien dat simpele paar-potentialen niet voldoende zijn om deze systemen te beschrijven.

Zoals we eerder vermeld hebben, verdelen we de polymeren over alle conformaties al naar gelang het Boltzmanngewicht van die conformaties. Sommige van die polymeerconformaties overlappen echter met zichzelf. In het echt zou een polymeer nooit in zo'n conformatie kunnen zitten. Met de SF-ZCV methode controleren we echter niet of conformaties overlappen. Aangezien in een compacte conformatie er vaker overlap is, worden deze conformaties daarom te zwaar meegeteld binnen de SF$\mathrm{ZCV}$ theorie. Daardoor is een polymeerkluwen in het SF-ZCV model minder gezwollen dan in het echt.

Er is echter ook een tegenovergesteld effect. Elke monomeer heeft een uitgesloten volume. Dat is het volume rond een monomeer waarbinnen zich geen andere monomeren kunnen bevinden. In echte polymeren overlappen de uitgesloten volumes van de aan elkaar gebonden monomeren elkaar. In het SF-ZCV model zijn de polymeren echter verspreid over vele conformaties. Hierdoor zullen de uitgesloten volumes van de segmenten elkaar veel minder overlappen en is er meer sterische hinder tussen verschillende polymeerketens.

Ik vroeg me af of we door de positie van een aantal segmenten vast te leggen deze fouten in het SF-ZCV model voor een deel zouden kunnen verhelpen.

Hoewel de kans dat de segmenten, waarvan de positie is vastgelegd, elkaar zouden overlappen klein is, moeten alle conformaties langs deze segmenten. Hierdoor is de dichtheid van de polymeren rond deze segmenten hoog en zorgt de osmotische druk ervoor dat de segmenten elkaar dus effectief afstoten. Hierdoor zal de grootte van de kluwen po- 
lymeer dus toe moeten nemen. Tegelijkertijd neemt de repulsie tussen verschillende polymeerketens juist af. Doordat de dichtheid rond de segmenten waarvan de positie is vastgelegd hoger is dan de gemiddelde dichtheid moet de dichtheid verder weg lager zijn en is er dus netto minder repulsie tussen de ketens.

Om te testen of dit inderdaad zo was heb ik dendrimeren gesimuleerd. Dit zijn sterk vertakte polymeren waarin de uitgesloten volume interacties een groot effect hebben op de structuur. Een voorbeeld van zo'n dendrimeer is weergegeven in Figuur 1.2. Om te kijken of de combinatie van de Monte Carlo en de zelfconsistent veld methode inderdaad een verbetering is ten opzichte van de klassieke SF-ZCV methode, moeten we natuurlijk een referentiesysteem hebben waarmee we kunnen vergelijken. Ik heb hiervoor een model gebruikt waarbij de polymeersegmenten als harde bolletjes voorgesteld worden en met bindingen van een vaste lengte aan elkaar vastzitten. De hoeken tussen deze bindingen kunnen elke waarde aannemen zolang de bolletjes maar niet overlappen.

De dendrimeren hadden drie tot vijf polymeren per vertakkingspunt en maximaal 7 generaties(het aantal vertakkingspunten dat je tegenkomt vanuit het midden van het polymeer.) De blokken tussen de vertakkingspunten waren 20 of 50 segmenten lang. Om rekentijd te besparen kwam mijn begeleider met het idee om voor elk blok, tussen de knooppunten, een apart simulatievolume te gebruiken, zodat we de dichtheid van dit blok niet in het hele volume uit hoefden te rekenen.

De uitkomst van de simulaties was dat beide SF-ZCV modellen de grootte van de dendrimeren onderschatten. In tegenstelling tot het klassieke model, dat de grootte van de dendrimeren te snel toe liet nemen met een toenemend aantal generaties, gaf de nieuwe hybride wel de juiste schaling van de grootte van de dendrimeer. De klassieke SF-ZCV theorie gaf ook een verkeerde ruimtelijke verdeling van de eerste generatie knooppunten. Bij de andere modellen waren de polymeerblokken tussen het midden van de dendrimeer en de eerste knooppunten sterk gestrekt. De klassieke ZCV theorie voorspelde echter dat een deel van de polymeerblokken tussen het centrum en de eerste generatie knooppunten niet gestrekt zou zijn terwijl de rest juist maximaal gestrekt is. Dit is te zien in Figuur 5.6. Mogelijk komt dit doordat in het klassieke ZCV model het ook mogelijk is om een fractie van een dendrimeerarm te strekken. Er is in het centrum niet genoeg ruimte voor een hele 
dendrimeerarm maar waarschijnlijk wel genoeg voor een fractie van een arm. De nieuw ontwikkelde hybride gaf dus een betere beschrijving van de dendrimeren.

Met deze nieuwe methode kan de SF-ZCV theorie toegepast worden op nieuwe vraagstukken. Het model is echter niet geschikt voor het bestuderen van dynamica omdat de polymeren door elkaar heen kunnen gaan en de vakjes nog relatief groot zijn. Om de dynamica van dit soort systemen te kunnen bestuderen zijn simulatie technieken zoals Brownse dynamica en dissipatieve deeltjes dynamica nodig. Hierbij moeten polymeer segmenten of zelfs hele micellen als een deeltje gesimuleerd worden. Het ontwikkelen van de correcte interactie potentialen vormt echter nog een grote uitdaging voor de toekomst. 


\section{List of Publications}

\section{This Thesis}

Coarse-Grained Dendrimers in a Good Solvent; Comparison of Monte Carlo Simulations, Self-Consistent Field Theory and a Hybrid Modelling Strategy

J. Bergsma, F. A. M. Leermakers and J. van der Gucht

Submitted, 2018

A Hybrid Monte Carlo Self-Consistent Field Model of Physical Gels of associërende Polymers

J. Bergsma, F. A. M. Leermakers. J. M. Kleijn and J. van der Gucht Journal of Chemical Theory and Computation

Volume 14, 2018, Pages 6532-6534

Interactions between Nodes in a Physical Gel Network of Telechelic Polymers; Self-Consistent Field Calculations Beyond the Cell Model J. Bergsma, F. A. M. Leermakers and J. van der Gucht Physical Chemistry Chemical Physics

Volume 17, 2015, Pages 9001-9004

Hybrid Monte Carlo Self-Consistent Field Approach to Model a Thin Layer of a Polyelectrolyte Gel near an Adsorbing Surface

F. A. M. Leermakers, J. Bergsma and J. van der Gucht

The Journal of Physical Chemistry A

Volume 116, 2012, Pages 6574-6581

\section{Other Work}

Performance of Metal Alloys as Hydrogen Evolution Reaction Catalysts in a Microbial Electrolysis Cell

Adriaan W. Jeremiasse, Johan Bergsma, J. Mieke Kleijn, Michel Saakes, Cees J.N. Buisman, Martien Cohen Stuart and Hubertus V.M.Hamelers International Journal of Hydrogen Energy

Volume 36, Issue 17, 2011, Pages 10482-10489 
List of Publications 


\section{Bibliography}

[1] J. François, S. Maitre, M. Rawiso, D. Sarazin, G. Beinert, and F. Isel. Neutron and x-ray scattering studies of model hydrophobically end-capped poly(ethylene oxide) aqueous solutions at rest and under shear. Colloids and Surfaces A: Physicochemical and Engineering Aspects, 112:251-265, 1996.

[2] X.-X. Meng and W. B. Russel. Rheology of telechelic associative polymers in aqueous solutions. J. Rheol., 50:189-205, 2006.

[3] Atsushi Harada and Kazunori Kataoka. Formation of polyion complex micelles in an aqueous milieu from a pair of oppositelycharged block copolymers with poly(ethylene glycol) segments. Macromolecules, 28:5294-5299, 1995.

[4] M. Lemmers, J. Sprakel, I. K. Voets, J. van der Gucht, and M. A. Cohen Stuart. Multiresponsive reversible gels based on charge driven assembly. Angewandte Chemie, 122:720-723, 2010.

[5] J. Wang, M. A. Cohen Stuart, A. T. M. Marcelis, M. ColombDelsuc, S. Otto, and J.van der Gucht. Stable polymer micelles formed by metal coordination. Macromolecules, 45:7179-7185, 2012 .

[6] M. Gerth, M. Bohdan, R. Fokkink, J. van der Gucht, and J. Sprakel. Supramolecular assembly of self-healing nanocomposite hydrogels. Macromolecular Rapid Communications, 35:20652070, 2014.

[7] Małgorzata A. Bohdan. Supramolecular networks of telechelic polymers. PhD thesis, Graduate School VLAG Wageningen University, 2016.

[8] E. Michel, J. Appell, F. Molino, J. Kieffer, and G. Porte. Unstable flow and nonmonotonic flow curves of transient networks. $J$. Rheol., 45:1465-1477, 2001. 
[9] Joris Sprakel. Physics of associative polymers; bridging time and length scales. PhD thesis, Graduate School VLAG Wageningen University, 2009.

[10] A. J. de Graaf, I. I. Azevedo Próspero dos Santos, E. H. E. Pieters, D. T. S. Rijkers, C. F. van Nostrum, T. Vermonden, R. J. Kok, W. E. Hennink, and E. Mastrobattista. A micelle-shedding thermosensitive hydrogel as sustained release formulation. Journal of Controlled Release, 162:582-590, 2012.

[11] Jian Ping Gong. Why are double network hydrogels so tough? SoftMatter, 6(12):2583-2590, 2010.

[12] Wolf Harald Rombouts. Composite hydrogels of bio-inspired protein polymers. PhD thesis, Graduate School VLAG Wageningen University, 2015.

[13] Wolf H. Rombouts, Mathieu Colomb-Delsuc, Marc W. T. Werten, Sijbren Otto, Frits A. de Wolf, and Jasper van der Gucht. Enhanced rigidity and rupture strength of composite hydrogel networks of bio-inspired block copolymers. Soft Matter, 9(29):69366942, 2013.

[14] Wolf H. Rombouts, Daan W. de Kort, Thao T. H. Pham, Carlo P. M. van Mierlo, Marc W. T. Werten, Frits A. de Wolf, and Jasper van der Gucht. Reversible temperature-switching of hydrogel stiffness of coassembled, silk-collagen-like hydrogels. Biomacromolecules, 16(8):2506-2513, 2015.

[15] Roger Eckhardt. Stan Ulam, John Von Neumann, and the Monte Carlo method. Los Alamos Science, Special issue:131, 1987.

[16] N. Metropolis, A. W. Rosenbluth, M. N. Rosenbluth, A. H. Teller, and E. Teller. Equations of state calculations by fast computing machines. J. Chem. Phys., 21:1087-1092, 1953.

[17] W. K. Hastings. Monte Carlo sampling methods using Markov chains and their applications. Biometrika, 57(1):97-109, 1970. 
[18] Daan Frenkel and Berend Smit. Understanding Molecular Simulation: From algorithms to Applications. Computational Science: From Theory to Applications. Academic press, 2002.

[19] G. J. Fleer, M. A. Cohen Stuart, J. M. H. M. Scheutjens, T. Cosgrove, and B. Vincent. Polymers at Interfaces. Chapman \& Hall, London, 1993.

[20] J. M. H. M. Scheutjens and G. J. Fleer. Statistical theory of the adsorption of interacting chain molecules. 1. partition function, segment density distribution, and adsorption isotherms. The Journal of Physical Chemistry, 83:1619, 1979.

[21] J. M. H. M. Scheutjens and G. J. Fleer. Statistical theory of the adsorption of interacting chain molecules. 2. train, loop, and tail size distribution. J. Phys. Chem., 84:178, 1980.

[22] F. A. M. Leermakers, J. M. H. M. Scheutjens, and J. Lyklema. On the statistical thermodynamics of membrane formation. Biophysical Chemistry, 18:353-360, 1983.

[23] H. Pera, J.M. Kleijn, and F.A.M. Leermakers. Linking lipid architecture to bilayer structure and mechanics using self-consistent field modelling. J. Chem. Phys., 140:065102, 2014.

[24] O. A. Evers, J. M. H. M. Scheutjens, and G. J. Fleer. Statistical thermodynamics of block copolymer adsorption. 1. formulation of the model and results for the adsorbed layer structure. Macromolecules, 23(5221-5232), 1990.

[25] L.A. Meijer, F.A.M. Leermakers, and J. Lyklema. Self-consistentfield modeling of complex molecules with united atom detail in inhomogeneous systems. cyclic and branched foreign molecules in dimyristoylphosphatidylcholine membranes. J. Chem. Phys., 110:6560-6579, 1999.

[26] Oleg V. Rud, Alexey A. Polotsky, Torben Gillich, Oleg V. Borisov, Frans A. M. Leermakers, Marcus Textor, and Tatiana M. Birshtein. Dendritic spherical polymer brushes: Theory and selfconsistent field modeling. Macromolecules, 46:4651-4662, 2013. 
[27] Y. Lauw, F.A.M. Leermakers, M.A. Cohen Stuart, O.V. Borisov, and E.B. Zhulina. Coexistence of crew-cut and starlike spherical micelles composed of copolymers with an annealed polyelectrolyte block. Macromolecules, 39:3628-3641, 2006.

[28] T. Cosgrove, T. Heath, B. van Lent, F. Leermakers, and J. Scheutjens. Configuration of terminally attached chains at the solid/solvent interface self-consistent field theory and a Monte Carlo model. Macromolecules, 20:1692-1696, 1987.

[29] E.B. Zhulina and F.A.M. Leermakers. A self-consistent field analysis of the neurofilament brush with amino-acid resolution. Biophysical J., 93:1421-1430, 2007.

[30] Y. Lauw, F.A.M. Leermakers, and M.A. Cohen Stuart. Selfconsistent-field prediction for the persistence length of wormlike micelles of nonionic surfactants. J. Phys. Chem., 107:1091210918, 2003.

[31] J. Klein Wolterink, F.A.M. Leermakers, G.J. Fleer, L.K. Koopal, E.B. Zhulina, and O.V. Borisov. Screening in solutions of starbranched polyelectrolytes. Macromolecules, 32:2365-2377, 1999.

[32] C.C. Van der Linden, B. Van Lent, F.A.M. Leermakers, and G.J. Fleer. Adsorption of polymers on heterogeneous surfaces. Macromolecules, 27:1915-1921, 1994.

[33] M. Charlaganov, O. V. Borisov, and F. A. M. Leermakers. Modeling of triblock terpolymer micelles with a segregated corona. Macromolecules, 41:3668-3677, 2008.

[34] M. Mocan, M. Kamperman, and F.A.M. Leermakers. Microphase segregation of diblock copolymers studied by the self-consistent field theory of Scheutjens and Fleer. Polymers, 1:1, 2018.

[35] M. Parrinello R. Car. Unified approach for molecular dynamics and density-functional theory. Phys. Rev. Lett., 55:2471, 1985.

[36] M. Brunner, C. Bechinge1, W. Strepp, V. Lobaskin, and H. H. von Grünberg. Density-dependent pair interactions in 2d. Europhysics Letters, 58(6), 2002. 
[37] Evert Jan Meijer and Daan Frenkel. Colloids dispersed in polymer solutions. a computer simulation study. J. Chem. Phys., 100:6873-6887, 1994.

[38] P. Chakraborty Pascal L. R. van der Veeken and Herman P. van Leeuwen. Accumulation of humic acid in DET/DGT gels. Environ. Sci. Technol., 44:4253-4257, 2010.

[39] Pascal L. R. van der Veeken and Herman P. van Leeuwen. DGT/DET gel partition features of humic acid/metal species. Environ. Sci. Technol., 44(14):5523-5527, 2010.

[40] P.G. De Gennes. Scaling concepts in polymer physics. Cornell Univ. press, Ithaca NY, 1979.

[41] Nathan Clisby. Accurate estimate of the critical exponent $\nu$ for self-avoiding walks via a fast implementation of the pivot algorithm. Phys. Rev. Lett., 104(055702), 2010.

[42] R Guida and J Zinn-Justin. Critical exponents of the nvector model. Journal of Physics A: Mathematical and General, 31(40):8103, 1998.

[43] A. N. Semenov, J.-F. Joanny, and A. R. Khokhlov. Associating polymers: Equilibrium and linear viscoelasticity. Macromolecules, 28:1066-1075, 1995.

[44] J. Sprakel, N. A. M. Besseling, M. A. Cohen Stuart, and F. A. M. Leermakers. Phase behavior of flowerlike micelles in a SCF cell model. The European Physical Journal E, 25:163-173, 2008.

[45] M. Filali, R. Aznar, M. Svenson, G. Porte, and J. Appell. Swollen micelles plus hydrophobically modified hydrosoluble polymers in aqueous solutions: Decoration versus bridging. a small angle neutron scattering study. J. Phys. Chem. B, 103:7293-7301, 1999.

[46] T. Liu, D. Liang, L. Song, V. M. Nace, and B. Chu. Spatial opennetwork formed by mixed triblock copolymers as a new medium for double-stranded dna separation by capillary electrophoresis. Electrophoresis, 22:449-458, 2001. 
[47] D. C. Tuncaboylu, M. Sari, W. Oppermann, and O. Okay. Tough and self-healing hydrogels formed via hydrophobic interactions. Macromolecules, 44:4997-5005, 2011.

[48] J.-F. Berret and Y. Séréro. Evidence of shear-induced fluid fracture in telechelic polymer networks. Physical Review Letters, 87:048303, 2001.

[49] J. Sprakel, E. Spruijt, J. van der Gucht, J. T. Padding, and W. J. Briels. Failure-mode transition in transient polymer networks with particle-based simulations. Soft Matter, 5:4748-4756, 2009.

[50] J. M. H. M. Scheutjens and G. J. Fleer. Interaction between two adsorbed polymer layers. Macromolecules, 18:1882-1900, 1985.

[51] S. P. F. M. Roefs, J. M. H. M. Scheutjens, and F. A. M. Leermakers. Adsorption theory for polydisperse polymers. Macromolecules, 27:4810-4816, 1994.

[52] M. Charlaganov and F. A. M. Leermakers. Molecular modeling of intermolecular and intramolecular excluded volume interactions for polymers at interfaces. J. Chem. Phys., 131:244115, 2009.

[53] B. R. Postmus, F. A. M. Leermakers, and M. A. Cohen Stuart. Self-consistent field modeling of adsorption from polymer/surfactant mixtures. Langmuir, 24:6712-6720, 2008.

[54] F. A. M. Leermakers, C. M. Wijmans, and G. J. Fleer. On the structure of polymeric micelles: Self-consistent-field theory and universal properties for volume fraction profiles. Macromolecules, 28:3434-3443, 1995.

[55] P. J. Flory. Thermodynamics of high polymer solutions. J. Chem. Phys., 10:51-61, 1942.

[56] Alexander D. MacKerell Jr. Hwankyu Lee, Richard M. Venable and Richard W. Pastor. Molecular dynamics studies of polyethylene oxide and polyethylene. Biophysical Journal, 95:1590-1599, 2008 . 
Bibliography

[57] D. C. Liu and J. Nocedal. On the limited memory bfgs method for large scale optimization. Mathematical Programming, 45:503-528, 1989.

[58] M. Daoud and J. P. Cotton. Star shaped polymers : a model for the conformation and its concentration dependence. J. Physique, 43:531-538, 1982.

[59] C. M. Wijmans and E. B. Zhulina. Polymer brushes at curved surfaces. Macromolecules, 26:7214-7224, 1993.

[60] G. A. Vliegenthart and H. N. W. Lekkerkerker. Predicting the gas-liquid critical point from the second virial coefficient. Journal of Chemical Physics, 112:5364-5369, 2000.

[61] J. Buffle and H.P. van Leeuwen. IUPAC Environmental Analytical and Physical Chemistry Series Vol. 1: Environmental particles Vol.1. Lewis Publishers, Boca Raton, 1992.

[62] J. Buffle and H.P. van Leeuwen. IUPAC Environmental Analytical and Physical Chemistry Series Vol. 2: Environmental particles Vol. 2. Lewis Publishers, Boca Raton, 1993.

[63] German Muller. Heavy metal accumulation in river sedimentsresponse to environmental pollution. Am. Assoc. Pet. Geol. Bull., 57:1844, 1973.

[64] W. Davison and H. Zhang. In situ speciation measurements of trace components in natural waters using thin-film gels. Nature, 367:546-548, 1994.

[65] W. Davison, G. Fones, M. Harper, P. Teasdale, and H. Zhang. Dialysis, DET and DGT: In Situ diffusional techniques for studying water, sediments and soils. In: In Situ Monitoring of Aquatic Systems: Chemical Analysis and Speciation. John Wiley \& Sons, 2000 .

[66] G.R. Fones, W. Davison, O. Holby, B.B. Jorgensen, and Bo Thamdrup. High-resolution metal gradients measured by in situ DGT/DET deployment in black sea sediments using an autonomous benthic lander. Limnol. Oceanog., 46:982-988, 2001. 
[67] Hao Zhang and William Davison. Diffusional characteristics of hydrogels used in DGT and DET techniques. Anal Chim. Acta, 398:329-340, 1999.

[68] P.J. Flory. Principles of Polymer Chemistry. Cornell Univ. Press, Ithaca, New York, 1953.

[69] F.G. Donnan. Membranpotentiale bei vorhandensein von nicht dialysieren elektrolyte. ein veitrag zur physikalische chemishen physiologie. Z. Elektrochemie, 17:572, 1911.

[70] P. G. de Gennes. Conformations of polymers attached to an interface. Macromolecules, 13(5):1069-1075, 1980.

[71] F.A. Escobedo and J.J. de Pablo. Molecular simulation of polymeric networks and gels: phase behavior and swelling. Physics Reports, 318(3):85-112, 1999.

[72] Stefanie Schneider and Per Linse. Discontinuous volume transitions in cross-linked polyelectrolyte gels induced by short-range attractions and strong electrostatic coupling. Macromolecules, 37(10), 2004.

[73] Daniel P. Aalberts. Microscopic simulation of phase transition in interacting ionic gels. Journal of Chemical Physics, 104:4309, 1996.

[74] A. Martin-Molina M. Quesada-Pérez, J. G. Ibarra-Armenta. Computer simulations of thermo-shrinking polyelectrolyte gels. Journal of Chemical Physics, 135:094109, 2011.

[75] M.A. Cohen Stuart, G.J. Fleer, J. Lyklema, W. Norde, and J.M.H.M. Scheutjens. Adsorption of ions, polyelectrolytes and proteins. Adv. Colloid Interface Sci., 34:477-535, 1991.

[76] M.P. Allen and D.J. Tildesley. Computer simuation of liquids. Clarendon, Oxford, 1987.

[77] M.R. Hestenes and E Stiefel. Methods of conjugate gradients for solving linear systems. Journal of Research of the National Bureau of Standards, 49(6), 1952. 
Bibliography

[78] T. Steihaug R.S. Dembo. Truncated-Newton algorithms for largescale unconstrained optimization. Mathematical Programming, 29(2):190-212, 1983.

[79] S.F. Edwards. The statistical mechanics of polymers with excluded volume. Proceedings of the Physical Society, 85:613, 1965.

[80] L.P. Yezek and H.P. van Leeuwen. An electrokinetic characterization of low charge density cross-linked polyacrylamide gels. Journal of Colloid and Interface Science, 278:243-250, 2004.

[81] O.V. Borisov T.M. Birshtein, E.B. Zhulina. Temperatureconcentration diagram for a solution of star-branched macromolecules. Polymer, 27(7):1078-1086, 1986.

[82] P.-G. de Gennes. Polymer solutions near an interface. adsorption and depletion layers. Macromolecules, 14:1637-1644, 1981.

[83] L.P. Yezek and H.P. van Leeuwen. Donnan effects in the steadystate diffusion of metal ions through charged thin films. Langmuir, 21(23):10342-10347, 2005.

[84] H.P. van Leeuwen. personal communications.

[85] Q. T. Pham, W. B. Russel, J. C. Thibeault, and W. Lau. Micellar solutions of associative triblock copolymers: Entropic attraction and gas-liquid transition. Macromolecules, 32:2996-3005, 1999.

[86] J. Sprakel, E. Spruijt, M. A. Cohen Stuart, N. A. M. Besseling, M. P. Lettinga, and J. van der Gucht. Shear banding and rheochaos in associative polymer networks. Soft Matter, 4(8):1696-1705, 2008.

[87] P.G. Khalatur, A.R. Khokhlov, and D.A. Mologin. Simulation of self-associating polymer systems. i. shear-induced structural change. The Journal of Chemical Physics, 109(21):9602-9613, 1998.

[88] M. J. Cass, D. M. Heyes, R-L. Blanchard, and R. J. English. Simulations and experiments of self-associating telechelic polymer solutions. J. Phys.: Condens. Matter, 20:335103, 2008. 
[89] Laurent Feuz, Frans A. M. Leermakers, Marcus Textor, and Oleg Borisov. Bending rigidity and induced persistence length of molecular bottle brushes: A self-consistent-field theory. Macromolecules, 38:8891-8901, 2005.

[90] István Borzsák and András Baranyai. On the convergence of green's entropy expansion. Chemical Physics, 165:227-230, 1992.

[91] N. Dawass, P. Krüger, S. K. Schnell, D. Bedeaux, S. Kjelstrup, J. M. Simon, and T. J. H. Vlugt. Finite-size effects of KirkwoodBuff integrals from molecular simulations. Molecular Simulation, 44(7):599-612, 2018.

[92] Pritam Ganguly and Nico F. A. van der Vegt. Convergence of sampling Kirkwood-Buff integrals of aqueous solutions with molecular dynamics simulations. J. Chem. Theory Comput., 9:1347-1355, 2013.

[93] T. Boublik. Equations of state of hard body fluids. Mol. Phys., 59:371, 1986.

[94] H. Venohr, V. Fraaije, H. Strunk, and W. Borchard. Static and dynamic light scattering from aqueous poly(ethylene oxide) solutions. European Polymer Journal, 34:723-732, 1998.

[95] Emmanuel Beaudoin, Christine Gourier, Alain Lapp, and Jeanne François. Influence of salts on hydrophobically end-capped polyethylene oxides in aqueous solution. Macromol. Symp., 146:171-177, 1999.

[96] J. François, E. Beaudoin, and O. Borisov. Association of hydrophobically end-capped poly(ethylene oxide). 2. phase diagrams. Langmuir, 19:10011-10018, 2003.

[97] Athanassios Z. Panagiotopoulos. Direct determination of phase coexistence properties of fluids by Monte Carlo simulation in a new ensemble. Molecular Physics, 61:813-826, 1987.

[98] J.S. Klos and J. U. Sommer. Coarse grained simulations of neutral and charged dendrimers. Polymer Science, Ser. C, 55(1):125-153, 2013 . 
[99] Matthias Ballauff and Christos N. Likos. Dendrimers in solution: Insight from theory and simulation. Angew. Chem. Int. Ed., 43:2998-3020, 2004.

[100] Zheng Yu Chen and Shi-Min Cui. Monte Carlo simulations of star-burst dendrimers. Macromolecules, 29:7943-7952, 1996.

[101] J.S. Klos and J.-U. Sommer. Properties of dendrimers with flexible spacer-chains: A monte carlo study. Macromolecules, 42:48784886, 2009.

[102] J. Bergsma, F. A. M. Leermakers, and J. van der Gucht. Interactions between nodes in a physical gel network of telechelic polymers; self-consistent field calculations beyond the cell model. Physical Chemistry Chemical Physics, 17:9001-9014, 2015.

[103] J. Bergsma, F. A. M. Leermakers, J. M. Kleijn, and J. van der Gucht. A hybrid Monte Carlo self-consistent field model of physical gels of telechelic polymers. Journal of Chemical Theory and Computation, DOI: 10.1021/acs.jctc.7b01264, 2018.

[104] I.V. Ionova and E.A. Carter. Error vector choice in direct inversion in the iterative subspace method. J. Compt. Chem., 17:1836$1847,1996$.

[105] J. Klein Wolterink, J. van Male, M. Daoud, and O. V. Borisov. Starburst polyelectrolytes: Scaling and self-consistent-field theory. Macromolecules, 36:6624-6631, 2003.

[106] Joseph Rudnick and George Gaspari. Elements of the Random Walk. Cambridge University Press, 2004.

[107] J.S. Klos and J.-U. Sommer. Simulations of neutral and charged dendrimers in solvents of varying quality. Macromolecules, 46:3107-3117, 2013.

[108] Edward G. Timoshenko, Yuri A. Kuznetsov, and Ronan Connolly. Conformations of dendrimers in dilute solution. The Journal of Chemical Physics, 117:9050, 2002. 
[109] Alexey A. Polotsky, Torben Gillich, Oleg V. Borisov, Frans A. M. Leermakers, Marcus Textor, and Tatiana M. Birshtein. Dendritic versus linear polymer brushes: Self-consistent field modeling, scaling theory, and experiments. Macromolecules, 43:95559566, 2010.

[110] Holger Merlitz, Chen-Xu Wu, and Jens-Uwe Sommer. Starlike polymer brushes. Macromolecules, 44:7043, 2011.

[111] F. Uhlík, P. Košovan, Z. Limpouchová, K. Procházka, O.V. Borisov, and F.A.M. Leermakers. Modeling of ionization of conformatinos of starlike weak polyelectrolytes. Macromolecules, 47:4004-4016, 2014.

[112] Filip Uhlík, Peter Košovan, Ekaterina B. Zhulina, and Oleg V. Borisov. Charge-controlled nano-structuring in partially collapsed star-shaped macromolecules. Soft Matter, 12:4846-4852, 2016.

[113] F. A. M. Leermakers, J. Bergsma, and J. van der Gucht. Hybrid Monte Carlo self-consistent field approach to model a thin layer of a polyelectrolyte gel near an adsorbing surface. The Journal of Physical Chemistry A, 116:6574-6581, 2012.

[114] Pascal L. R. van der Veeken, P. Chakraborty, and Herman P. van Leeuwen. Accumulation of humic acid in DET/DGT gels. Environmental Science and Technology, 44(11):4253-4257, 2010.

[115] Pascal L. R. van der Veeken, José P. Pinheiro, and Herman P. van Leeuwen. Metal speciation by DGT/DET in colloidal complex systems. Environmental Science and Technology, 42:8835-8840, 2008 .

[116] Pavel G. Khalatur, Alexei R. Khokhlov, Julia N. Kovalenko, and Dmitrii A. Mologin. Molecular dynamics study of the solution of semiflexible telechelic polymer chains with strongly associating end-groups. The Journal of Chemical Physics, 110(12):6039-6049, 1999 .

[117] H. Bagger-Jörgensen, L. Coppola, K. Thuresson, U. Olsson, and K. Mortensen. Phase behavior, microstructure, and dynamics 
Bibliography

in a nonionic microemulsion on addition of hydrophobically endcapped poly(ethylene oxide). Langmuir, 13:4204-4218, 1997.

[118] Nikos Ch. Karayiannis, Ageliki E. Giannousaki, and Vlasis G. Mavrantzas. An advanced Monte Carlo method for the equilibration of model long-chain branched polymers with a welldefined molecular architecture: Detailed atomistic simulation of an h-shaped polyethylene melt. Journal of Chemical Physics, 118(6):2451-2454, 2003.

[119] L.R. Dodd, T.D. Boone, and D.N. Theodorou. A concerted rotation algorithm for atomistic Monte Carlo simulation of polymer melts and glasses. Molecular Physics, 78:961-996, 1993.

[120] J.G.E.M. Fraaije B.A.C. van Vlimmeren. Calculation of noise distribution in mesoscopic dynamics models for phase separation of multicomponent complex fluids. Computer Physics Communication, 1996.

[121] A. V. Zvelindovsky, G. J. A. Sevink, B. A. C. van Vlimmeren, N. M. Maurits, and J. G. E. M. Fraaije. Three-dimensional mesoscale dynamics of block copolymers under shear: The dynamic density-functional approach. Phys. Rev. E, 57(5):R4879R4882, 1998.

[122] Pavel G. Khalatur and Alexei Khokhlov. Computer simulation of solutions of telechelic polymers with associating end groups. Macromolecular Theory Simulation, 5:877-899, 1996.

[123] T. Padding and W. J. Briels. Uncrossability constraints in mesoscopic polymer melt simulations: Non-rouse behavior of C120H242. J. Chem. Phys., 115:2846, 2001.

[124] W. K. den Otter A. van den Noort and W. J. Briels. Coarse graining of slow variables in dynamic simulations of soft matter. Europhys. Lett., 80(2):28003, 2007.

[125] Rafayel Petrosyan. Improved approximations for some polymer extension models. Rheologica Acta, 56(1):21-26, 2017. 
Bibliography 


\section{Acknowlegements}

After many years my thesis is finally here. I could however not have made it without the help of many others. Before naming any one in particular I would like to start by thanking those who have contributed to the many volunteer, open source and free software projects that I used during my PhD.

As I have done my PhD "extra thoroughly", I have had many many colleagues throughout these years. Too many to thank each one individually. I would however like to thank the group as a whole for housing me for all these years, organizing many activities and the coziness I experienced within the group. I would like to thank my supervisor, Jasper, for giving me the opportunity to do my thesis at PCC and his help with improving the writing of my articles. Frans, thank you, for always having an open door, for your patience (I was not always the easiest $\mathrm{PhD}$ student to work with), the many discussions we had about the self consistent field theory and the hours we spend together sifting through the code to find bugs. I would like to thank my room mates for the nice working atmosphere and the pleasant conversations, especially Sabine. It was nice to have you as my teaching buddy and I would like to thank you for organizing the PhD trip to California.

Even though, I did not use the RAPID program in the end, I would like to thank Wouter den Otter for receiving me in Twente and showing me how the RAPID program works. Dear Frans, Hanne, Mieke, Jasper and Marleen, thank you for believing that I would bring this PhD to a good end, even when I did not always do so myself.

I would like to thank the housemates I have had during these years. It was always very nice to come home after a day of work and find someone to discuss the day with over dinner. I would especially like to thank Roel, Merel and Arnold for the many discussions we have had about my $\mathrm{PhD}$.

Finally I would like to thank my parents, Jan and Petra. Without them standing up for me and their support during my study Molecular Sciences I would never have been in the position to start my PhD. 
Acknowlegements 


\section{Dankwoord}

$\mathrm{Na}$ vele jaren is mijn thesis eindelijk af. Ik zou het echter niet afgemaakt kunnen hebben zonder hulp van vele anderen. Om te beginen bedank ik de degenen die bijgedragen hebben aan alle open source software en de internetvraagbaken die ik tijdens mijn $\mathrm{PhD}$ gebruikt heb.

Aangezien ik mijn PhD "extra grondig" gedaan heb, heb ik door de jaren heen heel veel collega's gehad. Teveel om iedereen persoonlijk te bedanken. Ik wil de groep echter bedanken dat ze mij al die jaren een plek geboden hebben, voor alle activiteiten die er georganiseerd zijn en de gezelligheid sfeer. Ik wil mijn begeleider Jasper bedanken dat hij mij de kans heeft gegeven om mijn promotieonderzoek bij de Physical Chemistry and Soft Matter groep te doen en zijn hulp bij het verbeteren van mijn artikelen. Frans, dank je dat jouw deur altijd open stond, voor jouw geduld(ik was niet altijd de makkelijkste promovendus.), de vele discussies die we hadden over de zelfconsistent veld theorie en de uren die we samen achter de computer door brachten om de fouten in onze computer programma's op te sporen. Ik wil mijn kamer genoten bedanken voor de goede sfeer en de fijne gesprekken. Sabine, het was fijn om jou als maatje te hebben bij het geven van onderwijs en nog bedankt voor het organiseren van de reis naar Californië. Hoewel ik de RAPID methode uiteindelijk niet gebruikt heb, wil ik Wouter den Otter toch bedanken dat hij me in Twente ontvangen heeft en mij heeft laten zien hoe het programma werkt. Beste Frans, Hanne, Mieke, Jasper en Marleen, bedankt dat jullie erin zijn blijven geloven dat ik deze $\mathrm{PhD}$ tot een goed einde zou brengen, zelfs als ik dat zelf niet altijd deed.

Ik wil de huisgenoten die ik door de jaren heen gehad heb bedanken. Het was altijd fijn om, als ik na een dag werken thuis kwam, iemand te vinden waarmee ik tijdens het avondeten de dag kon bespreken. Ik wil Roel, Merel en Arnold bedanken voor de vele discussies die we gehad hebben over mijn promotie.

Tot slot wil ik mijn ouders, Jan en Petra, bedanken. Als zij niet voor mij opgekomen waren en mij gesteund hadden tijdens mijn studie had ik nooit aan mijn promotie onderzoek kunnen beginnen. 
Dankwoord 


\section{About the Author}

Johan Bergsma was born on the 10th of July 1982 in Dordrecht. He did his secondary eduction at the Insula College, where he graduated in 2000. During this time he developed a broad interest in the natural sciences and partook in the national Physics, Chemistry, Mathematics and Biology Olympics, reaching top 100 in the Mathematics Olympics twice and top 20 in both the Physics and Biology Olympics.

After finishing his secondary eduction he started the study Molecular Sciences at Wageningen University, with specializations in Physical Chemistry and Physical Biology.

He did his first master thesis at the Biophysics group where he worked on methods to measure the water flow in plants using non spatially resolved NMR measurements.

His second master thesis was executed at the Physical Chemistry and Colloid Science group under supervision of Adriaan Jeremiasse and Mieke Kleijn. This thesis was done in collaboration with Wetsus, a research institute for sustainable water technology. He made and investigated metal alloys that could catalyse the hydrogen forming reaction in microbial electrolysis cells. In these cells microorganisms growing on an electrode break down organic molecules into protons electrons and carbon dioxide. By applying a small voltage these protons and electrons can be recombined at the other electrode to form hydrogen gas.

He did his internship, supervised by Anna Pavlova and Evert Jan Meijer, at the Computational Chemistry Group at the University of Amsterdam, where he studied how a rhodium based complex catalysed the hydrogen transfer reaction using ab initio calculations.

Next to his study he did many activities. During his first year in Wageningen he organized activities with study association Alchimica and started volunteering at biological vegetarian restaurant Zeezicht. In his second year he became a member of youth association Unitas where he undertook many activities. He was a member of the film committee, managed the bar and designed and printed posters. In 2004 he jointed the board for a year as treasurer. 
In 2011 he started working on his Phd at Physical Chemistry and Soft Matter at Wageningen Univeristy. In this period he also started working as a volunteer at the local art house cinema Movie-W. At first he sold tickets and operated the projection equipment. Later he joined the board focussing on finances. 


\section{Overview of Completed Training Activities}

\section{Discipline Specific Activities}

\section{Activity}

Han sur Lesse Winterschool Statics 13th European School on Rheology 10th Soft Matter Meeting Utrecht 11th Soft Matter Meeting Eindhoven 12th Soft Matter Meeting Amsterdam MolSim: Understanding Molecular Simulation *

13th Soft Matter Meeting Wageningen Han sur Lesse Winterschool Dynamics RPK-B Polymer Physics

Surface and Soft Matter Rheology for Food Applications

Physics at FOM Conference

Physics at FOM Conference

4th Dutch Molecular Dynamics Day ${ }^{\dagger}$ 21th Soft Matter Meeting Wageningen 16th IACIS Conference Rotterdam * $\ddagger$

$\begin{array}{lc}\text { Graduate School/ } & \text { Year } \\ \text { Institute } & 2011 \\ & 2011 \\ \text { Universiteit Leuven } & 2011 \\ & 2011 \\ & 2012 \\ & 2012 \\ \text { Holland Research School } & \\ \text { of Molecular Chemistry } & 2012 \\ & 2012 \\ & 2013 \\ \text { PTG/e BV } & 2013 \\ & \\ & 2014 \\ \text { NWO } & 2015 \\ \text { NWO } & 2015 \\ & 2016 \\ \text { IACIS } & 2018\end{array}$

\footnotetext{
${ }^{*}$ Poster presentation

${ }^{\dagger}$ Oral presentation

${ }^{\ddagger}$ Soundbyte
} 


\section{General courses}

\begin{tabular}{|c|c|c|}
\hline Activity & $\begin{array}{l}\text { Graduate School/ } \\
\text { Institute }\end{array}$ & Year \\
\hline Mini symposium: & Wageningen UR Library & 2011 \\
\hline \multicolumn{3}{|l|}{ How to write a world-class paper } \\
\hline Competence Assessment & WGS & 2011 \\
\hline Introductory Comsol course & & 2012 \\
\hline $\begin{array}{l}\text { Techniques for Writing and Pre- } \\
\text { senting a Scientific Paper }\end{array}$ & WGS & 2013 \\
\hline $\begin{array}{l}\text { Interpersonal Communication } \\
\text { for PhD Students }\end{array}$ & WGS & 2013 \\
\hline $\begin{array}{l}\text { Share your chemical research } \\
\text { with a broad audience }\end{array}$ & De perskamer & 2013 \\
\hline Coaching traject & Wageningen Career Support & 2014 \\
\hline Introductory Matlab course & MathWorks & 2014 \\
\hline Efficient Writing Strategies & Wageningen in'to Languages & 2014 \\
\hline Intro to Parallel Programming & Udacity & 2015 \\
\hline Career Orientation & WGS & 2015 \\
\hline
\end{tabular}

\section{Optionals}

\section{Activity}

Year

International excursion of the Physical Chemistry and

Colloid Science Laboratory, California $\mathrm{USA}^{\dagger}$

Advanced soft matter PCC-31806

\footnotetext{
${ }^{\dagger}$ Oral presentation
} 
This research was funded by NWO, The Netherlands Organization for Scientific Research.

The image on the front cover shows a stereoscopic image of a polymer gel simulated with the Monte Carlo SCF hybrid model as described in chapter 4 The simulation parameters were: 5 polymers per node, a $\chi$ interaction parameter of 0.5 , a middle block length of 50 segments and polymer volume fraction of 0.125 . The polymer density goes from orange (high density) to translucent blue(low density). The nodes are coloured orange as the polymer density is highest in this region.

On the rear cover stereoscopic images of three different models, of a dendrimer with 5 chains per branch point, 3 generations and a spacer length of 20 segments, are shown. On top is the spherically symmetric cell model, calculated with the SF-SCF theory. In the middle is the new hybrid model. The branch points are clearly visible as orange crosses. On the bottom is a freely jointed bead chain model. The end-groups are coloured white, the branch points are red and the spacers are blue. These different models are discussed in detail in chapter 5.

Cover Design and Layout by: Johan Bergsma

Printed by: proefschriftmaken.nl 

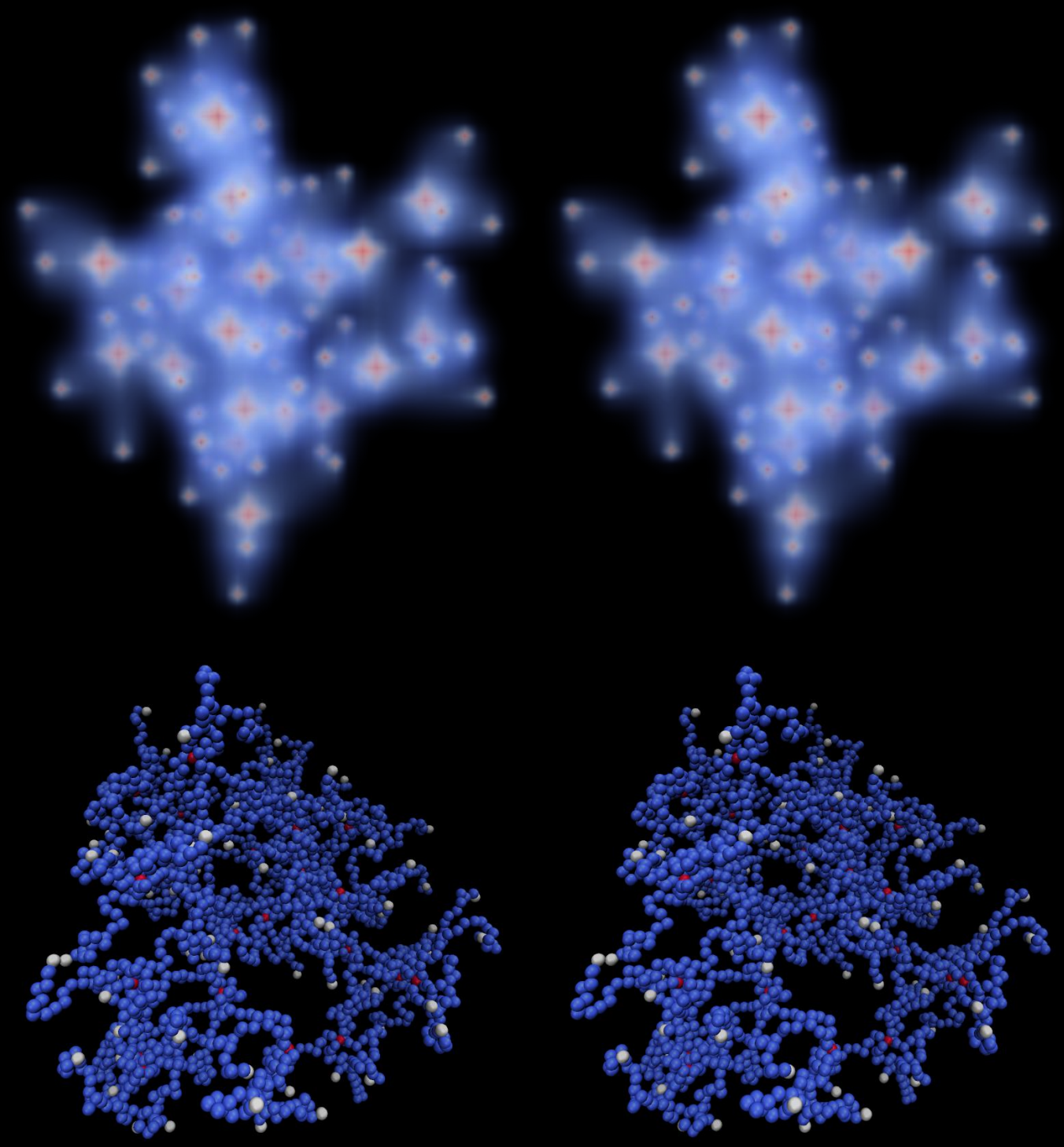